\title{
Mechanisms of Lung Ischemia-Reperfusion Injury and Donor Lung Rehabilitation by Ex Vivo Lung Perfusion
}

\author{
Matthew L. Stone, MD \\ Knoxville, Tennessee
}

Bachelor of Arts, College Scholars, Class of 2004, The University of Tennessee Doctor of Medicine, Class of 2008, ETSU James H. Quillen College of Medicine

A thesis presented to the Graduate Faculty of the University of Virginia in Candidacy for the Degree of Doctor of Philosophy

Department of Molecular Physiology and Biological Physics

\section{University of Virginia}

May 2016

PhD Committee Chair: Mentors:

PhD Committee:
Avril Somlyo, PhD, Professor Victor Laubach, PhD, Professor Irving Kron, MD, Professor Coleen McNamara, MD, Professor Mark Okusa, MD, Professor Craig Slingluff, MD, Professor 
To my parents, Dan and Sylvia Stone, for their faithful support. 


\section{Table of Contents}

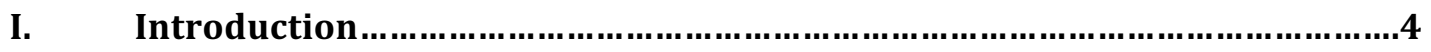

a. Current State of Lung Transplantation

b. Rationale and Objectives for Research

c. Thesis Objective and Thought Progression

II. Immunologic Mechanisms of Lung IR Injury

a. End Effector Cells Responsible for Lung IR Injury Pathogenesis

b. Role of Dendritic Cells and Macrophages in Lung IR Injury

c. Contributions of IL-12 and IL-23 in Lung IR Injury

III. Pharmacologic Targets for Lung IR Injury Prevention

a. Sphingosine Receptor Expression and Targeted Therapies

b. S1P Agonism as a Protective Strategy for IR Injury Prevention

c. S1PR1 Agonism as Principal Determinant of Pharmacologic Success

IV. Ex Vivo Lung Perfusion (EVLP) as a Strategy for Donor Lung Assessment and Rehabilitation.

a. Adenosine Receptor Targeted Therapies for IR Injury Prevention

b. Novel Murine EVLP Model Established A2A Receptor Agonism as Promising Strategy for Donor Lung Rehabilitation

c. Delayed Versus Immediate Perfusion Results in Superior Lung Function

d. EVLP and A2A Receptor Agonism Provides a Promising Strategy for Donor Lung Rehabilitation in a Pre-Clinical Model

e. Current Clinical Trial Evaluating Role of EVLP and A2A Receptor Agonism in Donor Lung Treatment for Rehabilitation

V. Conclusions and Future Directives

a. Targeted Immunologic Therapies for IR Injury Prevention

b. EVLP and A2A Agonism for Donor Lung Assessment, Treatment, and Rehabilitation

VI. Acknowledgements. 


\section{Introduction}

Lung transplantation remains the only curative hope for many with end stage pulmonary disease yet outcomes remain poor. As of 2013, nearly 11,000 lung transplant recipients were living in the United States[1]. Success of lung transplantation is significantly limited by ischemia-reperfusion (IR) injury following graft implantation, predisposing patients to primary graft dysfunction (failure of allograft within 72 hours of transplantation) and chronic graft rejection (bronchiolitis obliterans) following transplantation[2]. In addition to poor graft outcomes following transplantation, the inherent threat of IR injury has also resulted in a significant donor organ shortage, with limited acceptance of marginal heart-beating donor and non-heart-beating donor lungs for attempted transplantation. Strategies for rehabilitation and assessment of donor lungs are limited and the potential for immunologic manipulation has yet to be explored.

Advancements over the past decade have established cellular mediators (e.g. activation of iNKT cells, neutrophils, endothelial cells) and cytokine signaling pathways involved in the pathophysiology of allograft IR injury[3-6]. These discoveries have inspired further research into the interplay between innate and adaptive immune responses to IR injury and graft acceptance, specifically inspiring the study of more proximal mediators of IR injury to allow efficient and effective strategies for targeted pharmacologic therapies. Thus, the focus of study was to identify proximal mediators of lung IR injury with targeted analysis of macrophage and dendritic cell populations within the lung, as these cell types are implicated in the early pathogenesis of IR injury in other organ systems and contribute to both the innate and adaptive immune responses within the lung[6-14].

In addition to defining immunologic mediators of IR injury, a need for novel strategies of donor lung rehabilitation and assessment has emerged to improve graft function and enable expansion of a limited donor organ pool for many awaiting lung 
transplantation. Thus, targeted immunologic therapies are explored as a rehabilitative treatment for donor lungs following cardiac death utilizing ex vivo lung perfusion (EVLP).

Such study provided a translational aim for immunotherapy in donor lung allograft treatment and assessment prior to transplantation.

\section{Scope of the Problem}

Ischemia-Reperfusion Injury

Significant scientific and surgical advancements have supported improved survival and quality of life for lung transplant recipients and have led to a 30 -fold increase in the number of lung transplant recipients worldwide since its inception following the first human lung transplant in $1963[15,16]$. Lung transplant continues to have the poorest outcomes of all solid organ transplants with a demonstrated 54\% 5-year and 7.9 year median survival (Figure 1)[17].

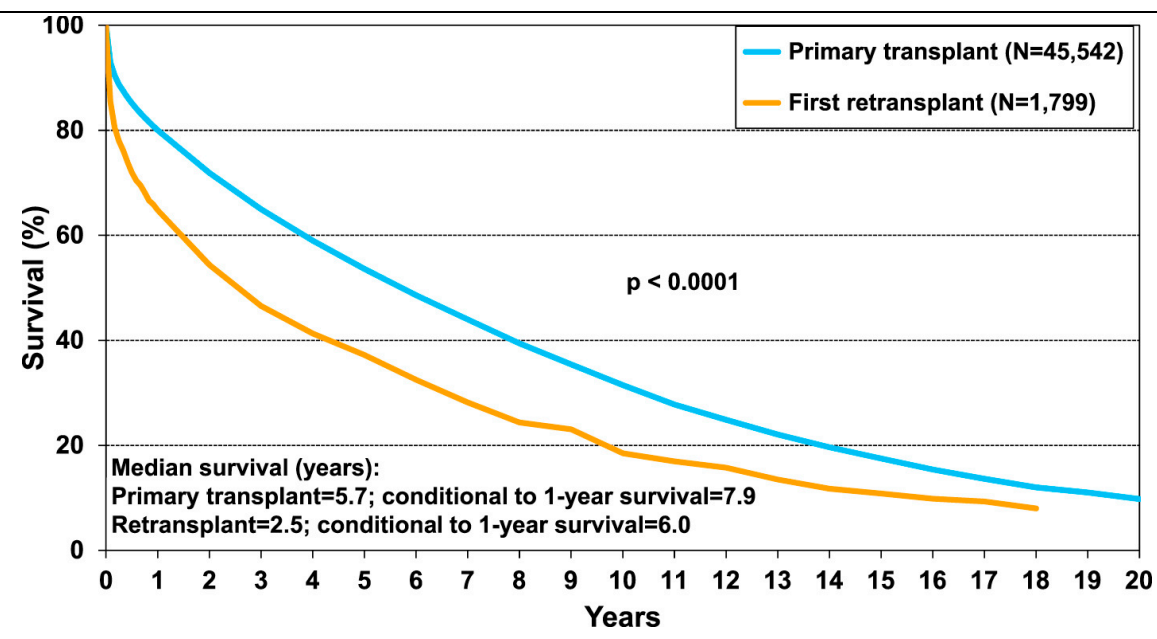

Figure 1. Adult lung transplant recipient Kaplan-Meier survival by transplant type (19902013)[17].

Current data demonstrate that IR injury and the resultant acute primary graft dysfunction (graft dysfunction within 30 days of transplantation) occur in $24 \%$ of patients[17]. Despite technical and research advancements over the past decade, primary graft dysfunction 
associated with allograft IR injury remains a significant threat to survival and long-term successful graft function (Figure 2)[17].

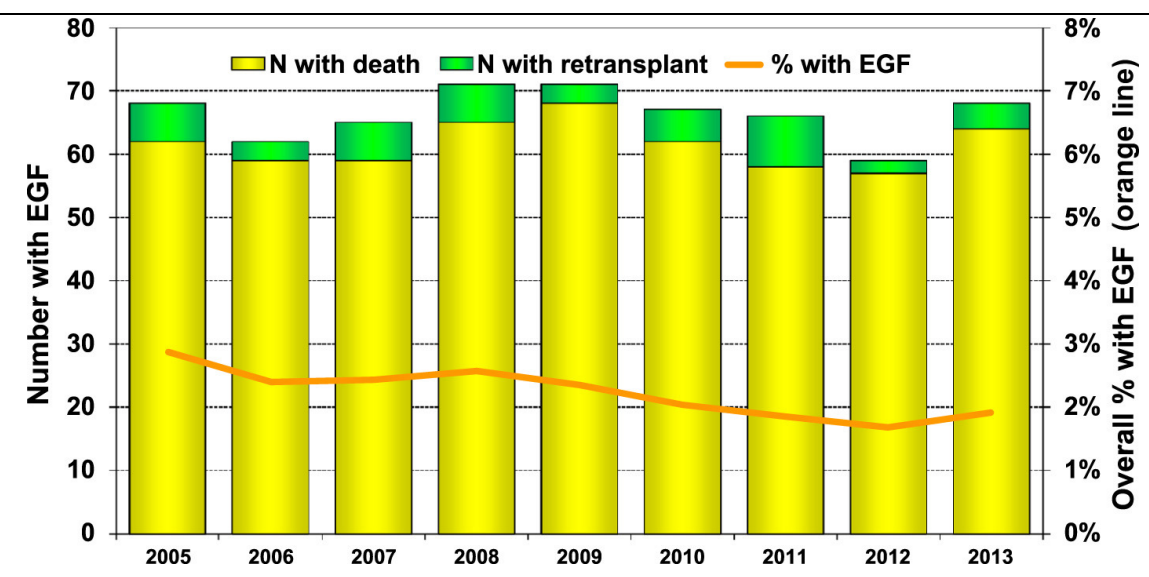

Figure 2. Number and percentage of adult primary lung transplants with early graft failure (EGF) by EGF type and by year (2005-2015). EGF defined as death or re-transplant associated with graft failure within 30 days after transplant[17].

Such early graft failure carried a 93\% risk of mortality and necessitated re-transplantation in $7 \%$ of patients in a worldwide analysis. Additionally, bronchiolitis obliterans is estimated to develop in $50 \%$ of primary adult lung transplant recipients within 5 years and in $76 \%$ of patients 10 years post-transplant, with both primary and late graft failure significantly increasing risk of mortality[17]. Collectively, these international data demonstrate that lung transplant outcomes remain poor and that IR injury is a primary threat to the success of this treatment strategy for end-stage pulmonary disease. In an era of rapidly evolving technologic innovation and pharmacologic targeting, a disparate level of advancement has occurred in lung transplantation. Thus, the focus of study was to develop reliable and reproducible models for lung IR injury and to advance the study of novel pharmacologic targets and organ perfusion techniques for improved allograft function and assessment prior to recipient implantation with the hope of improving recipient survival and quality of life post-transplantation. 


\section{Limited Donor Organ Availability}

There are currently 1,521 waiting list candidates for lung transplantation, with each facing an $18.8 \%(n=303 / 1,616)$ waiting list mortality secondary to decreased donor organ availability (Figure 3). Despite scientific and clinical advancements, donor lung rehabilitation and pre-transplantation allograft evaluation techniques remain limited with over $80 \%$ of potential donor lungs being injured and therefore considered not suitable for transplantation[18].

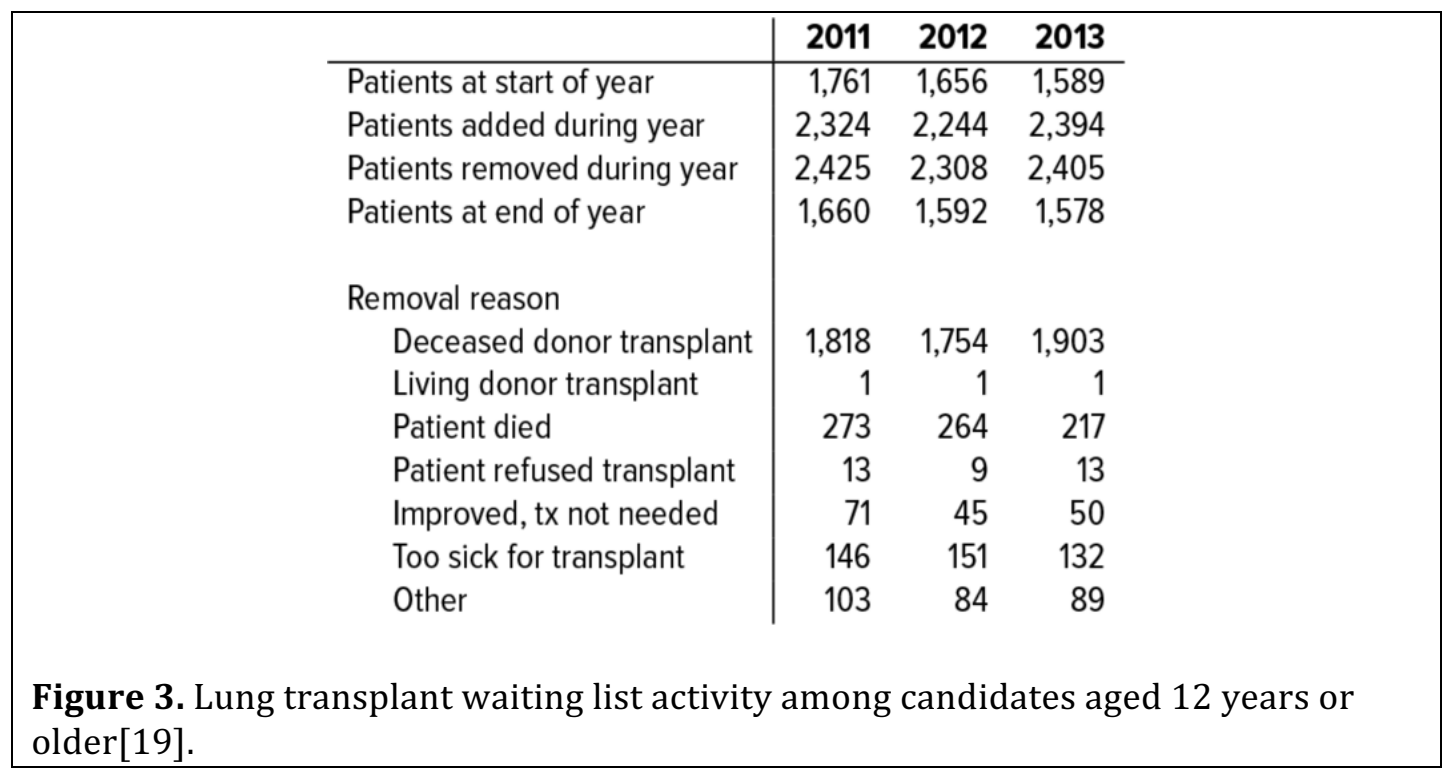

Recent reports from the International Society for Heart and Lung Transplantation (ISHLT) have demonstrated promise in the utilization of lungs following donation after circulatory death (DCD)[20]. This promising strategy represents a divergence from traditional donation after brain death (DBD) and has inspired the development of novel perfusion and preservation techniques, most notably ex vivo lung perfusion (EVLP) for donor lung rehabilitation and assessment. Such techniques offer the potential recovery and utilization of lungs that would have been rejected by traditional measures of allograft usability, while also offering a technique for donor lung treatment and assessment prior to recipient implantation. EVLP, as initially described by Cypel et al., provides physiologic 
normothermic acellular perfusion and rehabilitative ventilation to donor lungs during the preservation period[21]. This technique has demonstrated non-inferiority in the rehabilitation of previously rejected high-risk donor lungs for transplantation when compared to conventionally assessed donor lungs in a prospective randomized clinical trial and has increasing acceptance in the field of lung transplantation (Figure 4)[21]. Despite promising advancements in this technology for donor lung preservation, utilization of EVLP as a platform for pharmacologic agent delivery to rehabilitate lungs with extended warm ischemic times remains understudied and undefined. Thus, the purpose of study was to further evaluate the potential of EVLP as a strategy for donor lung rehabilitation utilizing established pharmacologic agents targeting the adenosine $2 \mathrm{~A}$ receptor in a novel murine non-heart-beating donation and EVLP model and a pre-clinical porcine model of lung transplantation. Following this progression of study, application to human lung transplantation is explored as a translational objective for donor pool expansion and donor lung assessment in clinical transplantation.

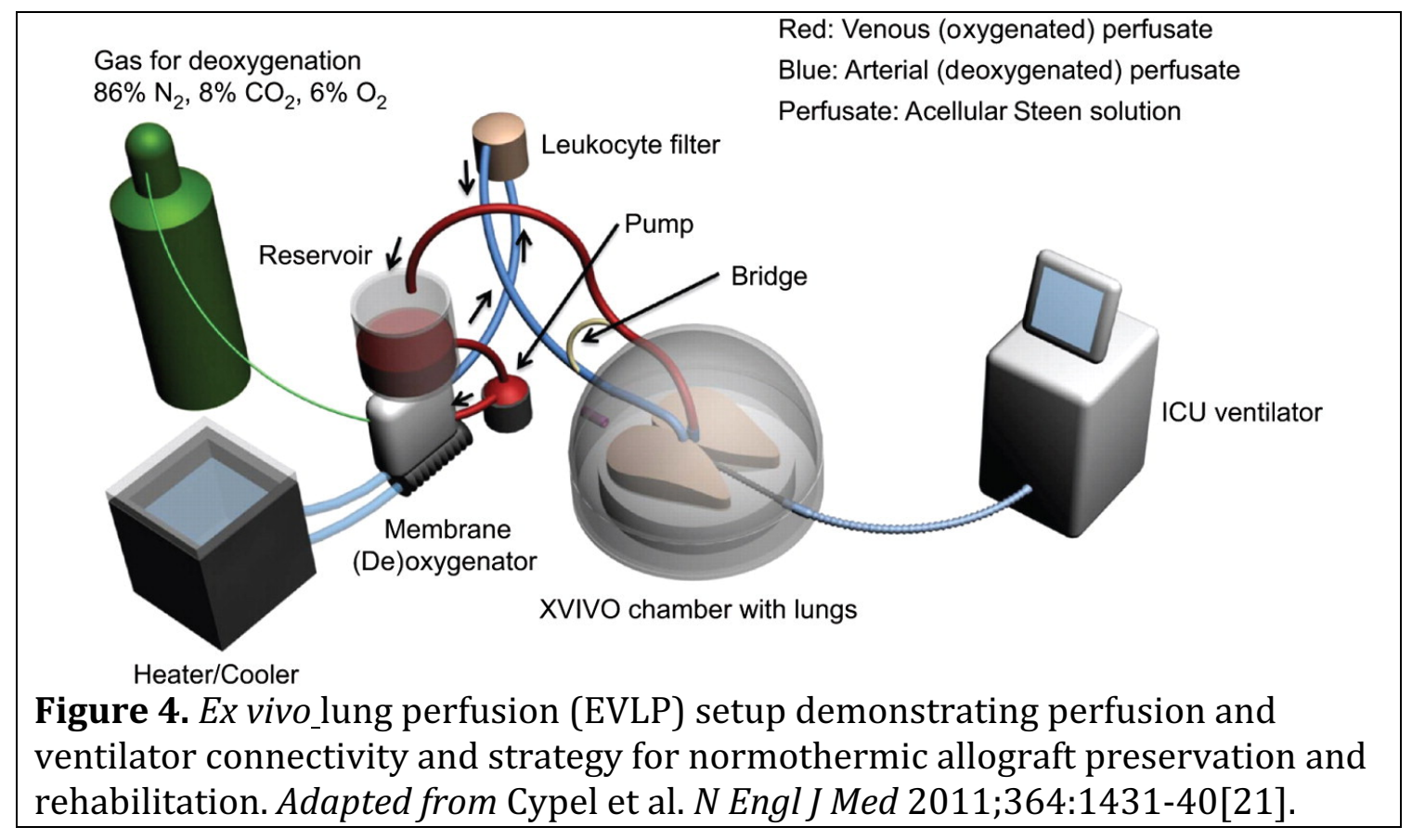




\section{Thesis Objective and Thought Progression}

The first of the two-part focus of study was to evaluate early cellular mediators implicated in the pathogenesis of lung IR injury. Specifically, we sought to evaluate the contributions of pulmonary dendritic cells and macrophages utilizing a novel in vivo targeted depletion model to determine the contributions of each in IR injury pathogenesis. This cell-based study was further expanded to determine the signaling mechanisms involved for these cell types in the initiation of pro-inflammatory pathways of lung injury and end effector cell activation. This study provided the framework for pharmacologic study with the adoption of sphingosine receptor targeted therapies, as modulation of sphingosine1-phosphate signaling is an established strategy for dendritic cell and macrophage signaling modulation[22]. Within this study we utilized a novel S1P pharmacologic agent, VPC-01091, and an established S1P agonist, FTY-720 to perform receptor-specific study of S1P analogs and the effect of each on IR injury pathogenesis. Additionally, we sought to determine whether the observed effects were the result of attenuated signaling or recruitment of iNKT cell end-effector cells. Cell-specific and pharmacologic studies were performed in an established in vivo murine model of lung IR injury.

The second focus of study was to evaluate the potential of EVLP in donor lung rehabilitation following cardiac death. We first developed a novel murine model of EVLP as a method for isolated perfusion and applied an established adenosine 2A receptor agonist, ATL-1223, as a strategy for donor lung treatment. This study provided a reproducible platform for the evaluation of EVLP-based therapies while also establishing rehabilitative potential for this pharmacologic perfusion strategy. The findings of this study provided a foundation for the preclinical translation of adenosine $2 \mathrm{~A}$ receptor agonist-based EVLP perfusion strategies within a porcine model of lung transplantation. Within this model we evaluated both the optimal timing and efficacy of EVLP following cardiac death. These 
studies provided a strategy for further translation into human clinical lung transplantation and herein we describe our initial experiences in human donor lung allograft rehabilitation. The collaborative findings of the presented studies provide a platform for further discussion and evaluation of implicated cell types in lung IR injury prevention and the responsible adoption of EVLP as a strategy for donor lung pharmacologic treatment and rehabilitation. 


\section{Immunologic Mechanisms of Lung Ischemia-Reperfusion (IR) Injury}

Cellular mechanisms of lung IR injury signaling are explored with specific attention to pulmonary dendritic cells and macrophages and the IL-12/IL-23 signaling pathways for $T$ cell activation. 
CD11c+ Dendritic Cells and Macrophages Mediate Lung Ischemia-Reperfusion Injury through IL-23/IL-12 Dependent Mechanisms

Matthew L. Stone, MD ${ }^{1}$, Ashish K. Sharma, MBBS PhD', Lucas Fernandez, MD MSc, Li Li, MD $\mathrm{PhD}^{2}$, Taeg S. Kim, PhD, Yunge Zhao, MD PhD³, Irving L. Kron, MD¹, Victor E. Laubach, PhD ${ }^{1}$

Departments of Surgery ${ }^{1}$, Medicine ${ }^{2}$, and Immunology 3 , University of Virginia, Charlottesville, Virginia, USA 


\section{Abstract}

Objective: Activated CD11c+ dendritic cells and macrophages participate in innate immunity and are established cell-mediators of the IL-23 and IL-12 pro-inflammatory cytokine axis. The purpose of this study was to test the hypothesis that CD11c+ cells, via induction of the IL-23/IL-12 axis, are an important component of the innate immune responses to lung IR injury.

Methods: A murine left hilar ligation model of lung IR injury was utilized that entailed 1 hour of ischemia and 2 hours of reperfusion. Bone marrow transplants (BMT) from CD11cand CD11b-diphtheria toxin receptor (DTR) mice to C57BL/6 wild-type (WT) recipient mice enabled the selective in vivo depletion of CD11c+ and CD11b+ cells by diphtheria toxin administration, respectively. Additionally, cytokine knockout mice (IL-23p19-/-, IL-12p40/-, and IL-12p35-/- were similarly compared. Pro-inflammatory cytokine expression was evaluated by ELISA of bronchoalveolar lavage fluid and gene expression in tissue via realtime PCR (RT-PCR). Neutrophil recruitment and activation were evaluated by immunohistologic staining and myeloperoxidase assessment.

Results: Compared to WT BMT mice, both CD11c-DTR and CD11b-DTR BMT groups exhibited significantly less pulmonary dysfunction after IR compared to the WT BMT IR injury control. In addition, CD11c-DTR BMT mice exhibited decreased inflammatory cytokine expression and neutrophil infiltration following lung IR in comparison to the WT BMT control. CD11c-DTR BMT mice also demonstrated decreased IL-23p19 gene expression following lung IR in comparison to the WT BMT IR control. IL-23p19-/- mice demonstrated significantly reduced functional IR injury compared to both WT controls. This protection was not afforded by knockout of either IL-12p40 or IL-12p35.

Conclusions: CD11c-DTR and CD11b-DTR mice allow selective depletion of dendritic cell and macrophage subsets within the lung. CD11c-DTR mice exhibit decreased lung IR injury compared to control, supporting a role for macrophage activation independent of dendritic cells in IR injury pathogenesis. Together, these data demonstrate that IL-23p19 production from macrophage and dendritic cell populations may provide a promising target for potential IR injury prevention. 


\section{Introduction}

Lung transplantation provides a life-sustaining hope for patients with end-stage pulmonary disease. Patient survival and graft function following lung transplantation, while improved, remain poor despite significant clinical and research advancements[23]. The primary etiology for both primary graft failure and late-term broncholitis obliterans following lung transplantation is lung IR injury[24]. Lung IR injury is estimated to occur in $22-37 \%$ of lung transplantation recipients[24-26]. Patients with primary graft dysfunction associated with IR injury demonstrate a 36\% increased risk of mortality within 30 days following lung transplantation as well as an increased risk of late-term bronchiolitis obliterans[2, 24]. Thus, a commitment to understanding immunologic mechanisms inherent to lung IR injury is needed, and such studies will support the development of directed therapies in lung transplantation to sustain the promise of improved outcomes. While the identification of effector cells within lung IR injury marks an accomplishment of the past decade, proximal mediators of innate immunity after IR remain ill-defined.

Effector cell mechanisms of lung IR injury most notably involve monocytes, CD4(+) T cells, neutrophils, and alveolar macrophages[3, 6, 27-29]. Dendritic cells and macrophages are principal cell types involved in both innate and adaptive immunity and characteristically comprise the CD11c+ population of cells within the lung[30,31]. A recent study has established that CD11c+ dendritic cells and macrophages exhibit chemokinemediated and directed recruitment to the lung in response to non-infectious airway inflammation[12]. Neutrophil-dendritic cell interactions have also recently been implicated in lung transplantation graft acceptance, with the demonstration that granulocyte-colony stimulating factor activates donor lung dendritic cells both in the stimulation of natural killer $\mathrm{T}$ cells and effector $\mathrm{T}$ cells in lung transplantation[32]. Additionally, host $\mathrm{T}$ cells have been found to cluster predominantly around lung-resident and donor-derived CD11c+ 
dendritic cells outside of secondary lymphoid organs following transplantation, identifying the dendritic cell as a link between the innate and adaptive responses to lung transplantation[33]. These findings support a principal role for both dendritic cells and macrophages in innate immunity, providing the foundation for further research to better define the contribution of each in the pathogenesis of early lung IR injury. The purpose of this study was to evaluate the contribution of CD11c+ dendritic cells and macrophages in the early innate immune response to lung IR injury and to analyze the potential contribution of the IL-23/IL-12 axis in effector cell activation and injury pathogenesis.

IL-12 and IL-23 are products of activated dendritic cells and macrophages and belong to the same cytokine family, sharing the p40 subunit yet demonstrating distinct biologic activities[34]. IL-12 is considered a principal activator of cell-mediated immunity with biologic activity only as a composite factor of p35 and p40 subunits, while the related IL-23 cytokine is distinguished by p19 and p40 subunit expression[34]. IL-12 and IL-23 provide a principal link between innate and adaptive immunity, determining Th1 and Th17 cell-directed responses[35]. The IL-12/IFN- $\gamma$ and IL-23/IL-17 pathways have been found to drive neutrophil migration and tissue injury in renal IR pathology[36]. These foundational principles both inspired and supported our independent and paired comparison of dendritic cells and macrophages and their contributions to the IL-23/IL-12 axis in lung IR injury.

\section{Methods}

\section{Study Design and Animals}

Bone marrow transplants (BMT) for each experimental group were necessitated due to neurologic complications associated with serial Isoflurane anesthesia doses, likely secondary to depletion of CD11c+ neuronal cells following toxin administration[37]. To limit any potential effect of BMT on outcomes following IR injury, all experimental groups 
underwent BMT. Three groups of wild-type (WT) C57BL/6 (Jackson Laboratory, Bar Harbor, ME) bone marrow chimera mice created from WT, CD11c-DTR (Jackson Laboratory, Bar Harbor, ME) and CD11b-DTR (Jackson Laboratory, Bar Harbor, ME) donors as previously described[5].

CD11c and CD11b transgenic (Tg) mice express a nonhuman primate diphtheria toxin receptor (DTR) under the control of a mouse CD11c or CD11b promoter (B6.FVBTg(Itgax-DTR/EGFP)57Lan/J; FVB-Tg(ITGAM-DTR/EGFR)34Lan/J). Diphtheria toxin administration allowed conditional depletion of CD11c+ (4ng/kg body weight intraperitoneally 12-24 hours prior to experimental IR injury) or CD11b+ cells in each mouse construct $(25 \mathrm{ng} / \mathrm{kg})[7,38,39]$.

Donor marrow was harvested under sterile conditions following euthanasia of each donor (male, 24-26g, age: 8-10 weeks), yielding approximately 50 million nucleated bone marrow cells per mouse. Recipient mice (male, 22-26g, age 6 weeks) were irradiated with 2 sequential doses of 6 Gy, each separated by 4 hours. Following the second irradiation, mice were anesthetized and injected with 2-4 x $10^{6} \mathrm{BM}$ cells via retro-orbital injection. A control mouse was assigned to each recipient group, with resultant mortality confirming efficacy of BM depletion by irradiation. Transplanted mice were housed for a 6 week minimum period for donor marrow reconstitution prior to experimentation. The completed chimera groups included: WT to WT, CD11c-DTR to WT, and CD11b-DTR to WT. Batf3-/- mice were also utilized for the study of CD103+ dendritic cell absence and the effect on lung injury[40, 41]. This study conformed to the Guide for the Care and Use of Laboratory Animals published by the National Institute of Health and was conducted under protocols approved by the University of Virginia's Institutional Animal Care and Use Committee.

Cytokine knockout comparison groups included IL-23p19-/-, IL12p40-/- and IL12p35-/- mice (n=5-8/group)(Jackson Laboratory, Bar Harbor, ME). Each of these 
knockout groups were on a C57BL6 background and thus were compared to a C57BL6 WT non-bone marrow transplanted control group.

In vivo Model of Lung IR Injury

An established in vivo model of IR was performed in each experimental group[4]. Briefly, mice were anesthetized with isoflurane inhalation, intubated, and ventilated at 120 strokes/min. with room air. Heparin was injected via the right external jugular vein $(20$ $\mathrm{U} / \mathrm{kg}$ ). A left anterolateral thoracotomy was performed at approximately the third intercostal space to allow access to the left pulmonary hilum. A 6-0 Prolene was passed around the hilum and the two ends of the suture were passed through PE-60 tubing. Surgical clip application secured the tubing in place, effectively occluding the pulmonary hilum during the ischemic period. Mice were recovered following analgesic administration (buprenorphine, $0.2 \mathrm{mg} / \mathrm{kg}$ ) and subjected to one-hour of ischemia prior to repeat anesthesia, intubation, and removal of the left lung hilum occlusion. Mice were then recovered from anesthesia and subjected to a two-hour reperfusion period. Sham groups underwent the same surgery without hilar occlusion.

\section{Measurements of Lung Function}

Pulmonary function analyses were conducted at the conclusion of the reperfusion period for each mouse on an isolated, buffer-perfusion apparatus (Hugo Sachs Elektronik, March-Huggstetten, Germany)[6]. Briefly, mice were anesthetized and maintained on intratracheal ventilation (rate: 100 strokes/min., tidal volume: $7 \mu \mathrm{L} / \mathrm{g}$ body weight, positive endexpiratory pressure: $2 \mathrm{cmH}_{2} 0$ ). Mice were exsanguinated by inferior caval transection, the pulmonary artery was cannulated through the right ventricle, and the left ventricle was tube vented through a small incision at the apex of the heart. The lungs were perfused at a constant flow of $60 \mu \mathrm{L} / \mathrm{g}$ body weight/minute with Krebs-Henseleit buffer. Following five 
minutes of equilibration, functional data were recorded for an additional ten minutes using PULMODYN data acquisition (Hugo Sachs Elektronik).

\section{Bronchoalveolar Lavage}

Following the completion of reperfusion or sham thoracotomy, the thoracic cavity was entered through a median sternotomy. The right lung hilum was occluded with a microclamp to allow isolated assessment of the left lung. The left lung was lavaged with two consecutive aspirates of the same sample through an anterior tracheotomy $(0.4 \mathrm{~mL})$. An average of $0.3 \mathrm{~mL}$ was retrieved by lavage and samples were immediately centrifuged at $4{ }^{\circ} \mathrm{C}$ (1500rpm for $8 \mathrm{~min}$.$) and the supernatant was stored at -80^{\circ} \mathrm{C}$.

Cytokine and Myeloperoxidase Measurements

The levels of pro-inflammatory cytokines in bronchoalveolar lavage samples were quantified using a Bio-Plex multiplex cytokine panel (Bio-Rad Laboratories, Hercules, CA). Myeloperoxidase (MPO) levels were measured within bronchoalveolar lavage samples using a murine MPO ELISA kit (Cell Sciences, Canton, MA).

Immunohistochemistry and Neutrophil Counting

Lungs were inflation-fixed under $20 \mathrm{~cm} \mathrm{H}_{2} 0$ pressure with $4 \%$ paraformaldehyde at $4^{\circ} \mathrm{C}$ for 15 hours and then placed in $70 \% \mathrm{EtOH}$ for paraffin embedding. One lung tissue slide from each experimental mouse ( $n=5$ /group) was generated for neutrophil staining as previously described[42]. Rat anti-mouse neutrophil (GR1.1, Santa Cruz, Biotechnology) primary antibody immunostaining was performed using Vectastain $\mathrm{ABC}$ kit (Vector Laboratories, Burlingame, CA) and alkaline phosphatase-conjugated anti-rat IgG provided the secondary antibody. The signals were detected using Fast-Red (Sigma Aldrich, St. Louis, MO). Purified normal rat immunoglobulin G (eBiosceince Inc., San Diego, CA) was used as the negative control. Each section was then counterstained with hematoxylin. Each lung section ( 1 slide/mouse) was used for semi-quantitative cell counting by a blinded 
investigator. Neutrophils were counted from three semi-standardized quadrants at 400x magnification and averaged for statistical analysis. All photos were generated under an Olympus BX51 microscope equipped with an Olympus DP70 digital camera (Minneapolis, $\mathrm{MN})$.

Real-time PCR (RT-PCR)

Real-time PCR was performed as described by our institutional collaborators on whole lung tissue [36]. Primer sequences for the p19 subunit were as follows: CAACTTCACACCTCCCTAC (forward), CCACTGCTGACTAGAACT (reverse). RT-PCR was performed on cDNA using iScript 1-step RT-PCR kit with SYBR Green (Bio-Rad) and samples normalized to GAPDH. Denaturation, annealing, elongation, incubation, and holding steps were conducted as previously described[43].

Flow Cytometry of Dendritic Cell and Macrophage Populations

Lungs were harvested and subjected to collagenase digestion followed by passage through a $40-\mu \mathrm{m}$ cell strainer (BD Falcon) and centrifuged at $1500 \mathrm{rpm}$ for 8 minutes followed by washing with $0.5 \%$ BSA in PBS. Harvest for both CD11c-DTR and CD11b-DTR groups was performed at 12-24 hours following diphtheria toxin injection, corresponding to the timing for experimental IR injury experimentation as described. Samples were then washed in two cycles with T-cell buffer and RBC lysis was performed sequentially (RBC lysis buffer, eBioscence). Following resuspension into FACS buffer (eBioscience), Samples were subsequently blocked with anti-mouse CD16/CD32 (1 $\mathrm{\mu g} / \mathrm{mL}$; eBioscience per $10^{6}$ cells for 15 minutes at $4^{\circ} \mathrm{C}$ ) prior to surface labeling with V450 live-dead staining (Invitrogen). Cell suspensions were fixed with Cytofix/Cytoperm solution (BD Bioscience) for 20 minutes at $4^{\circ} \mathrm{C}$. Cell suspensions were resuspended in Perm/Wash buffer (BD Bioscience) and labeled with the following fluorochromes: CD45+ (APC-Cy7), CD11c+ (APC), MHC-II+ (FITC), B220 (PerCP Cy5), CD11b (PE Cy7), CD103 (PE), and Siglec F (PE). Dendritic cells were defined 
by CD45, CD11c, and MHC-II positivity with negative selection for B220 and sub-classified as CD11b+ or CD103+. Similarly, macrophages were defined by CD45, CD11c, and MHC-II positivity with negative selection for B220 and differentiated by Siglec-F positivity. These classification systems and flurochrome staining analyses were derived from previously performed studies of dendritic cell and macrophage subsets within the lung[44]. Isotype and single-color controls were sequentially performed. Flow cytometry analyses were performed as previously described and FACS data analyzed using Flowjo software 8.8[3]. Statistical Analysis

Experimental methodology and design were conducted to evaluate the null hypothesis that no significant differences exist between cell population depletion or cytokine knockout groups. Pairwise comparisons were performed for each parameter of lung injury utilizing an unpaired Student's $t$ test or Mann-Whitney $U$ test as appropriate with significance defined as a $p$ value $<0.05$.

\section{Results}

Macrophage and Dendritic Cell Subpopulations are Differentially Depleted in CD11c-DTR and CD11b-DTR Mice following Diphtheria Toxin Administration

Lungs of wild-type BMT mice contained both CD11b- and CD103-positive dendritic cell subsets following diphtheria toxin administration in addition to macrophages, as identified by Siglec-F positivity (Figure 1A). Diphtheria toxin administration to CD11c-DTR BMT mice resulted in the conditional depletion of CD103+ dendritic cells and macrophages with a persistence of CD11bhi dendritic cells (Figure 1B). Conversely, CD11b-DTR BMT mice were depleted of CD11 bhi dendritic cells with a persistence of macrophages and CD103+ dendritic cells (Figure 1C). BatF3-/- mice were depleted of solely CD103+ dendritic cells as previously shown (Figure not shown). 

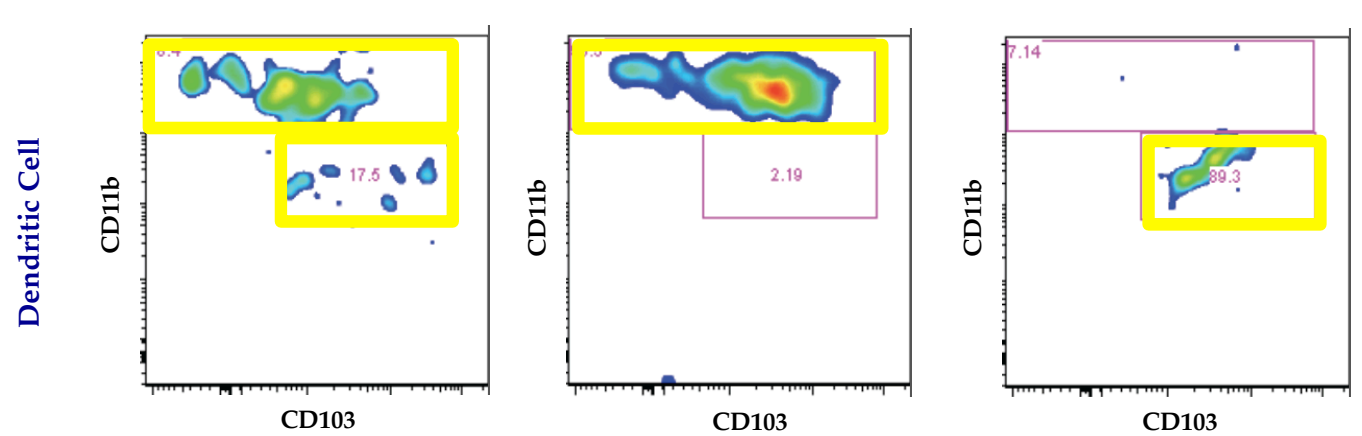

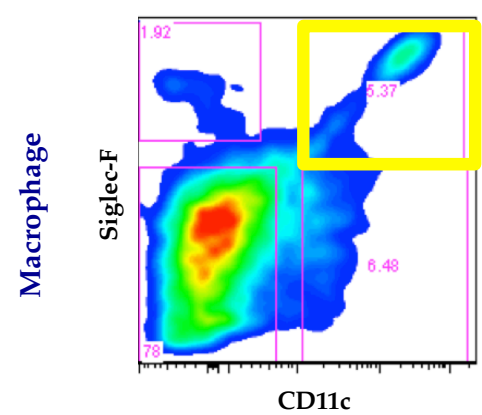

A. Wild Type

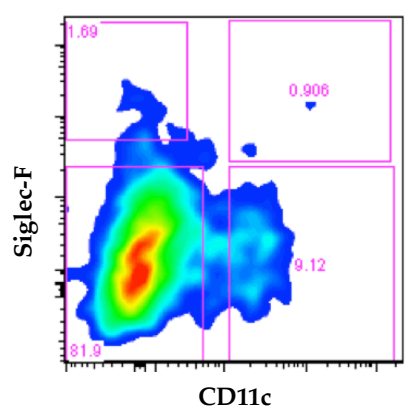

B. CD11c-DTR

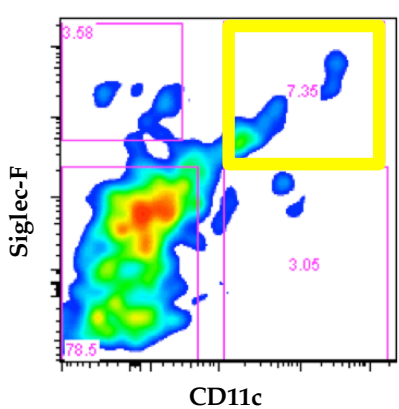

C. CD11b-DTR

Figure 1. Flow cytometry depletion images from respective mouse lungs demonstrating selective macrophage and dendritic cell depletion within each bone marrow transplant construct. A. Wild Type, C57Bl6; B. CD11c-DTR; C. CD11b-DTR.

Lung Function Following IR is Significantly Improved in CD11c-DTR and CD11b-DTR mice but not BatF3-/- Mice

Lung function was significantly worse in WT BMT mice after IR compared to sham (Figure 2A-B). CD11c-DTR and CD11b-DTR mice had significantly decreased levels of functional injury with increased pulmonary compliance and decreased pulmonary artery pressures compared to WT BMT controls. This attenuated injury was not observed in BatF3-/- mice as BatF3-/- mice demonstrated significantly increased airway resistance, decreased pulmonary compliance and increased pulmonary artery pressures compared to WT IR control (Figure 2C). 
A.

Airway Resistance

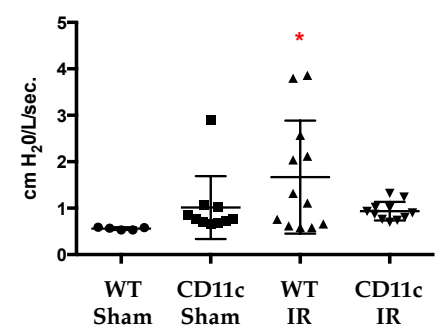

Pulmonary Compliance

Pulmonary Artery Pressure
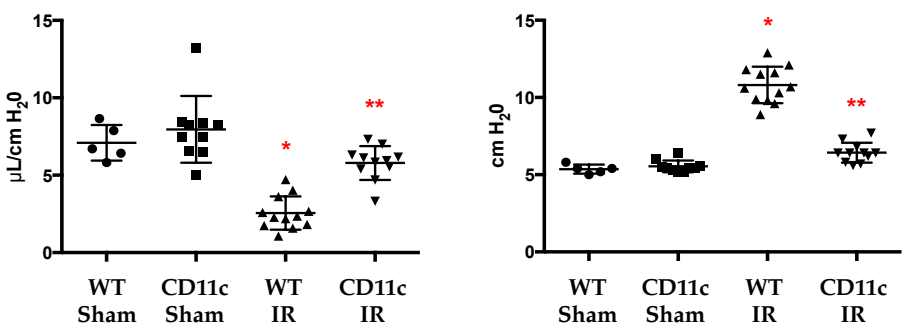

B.
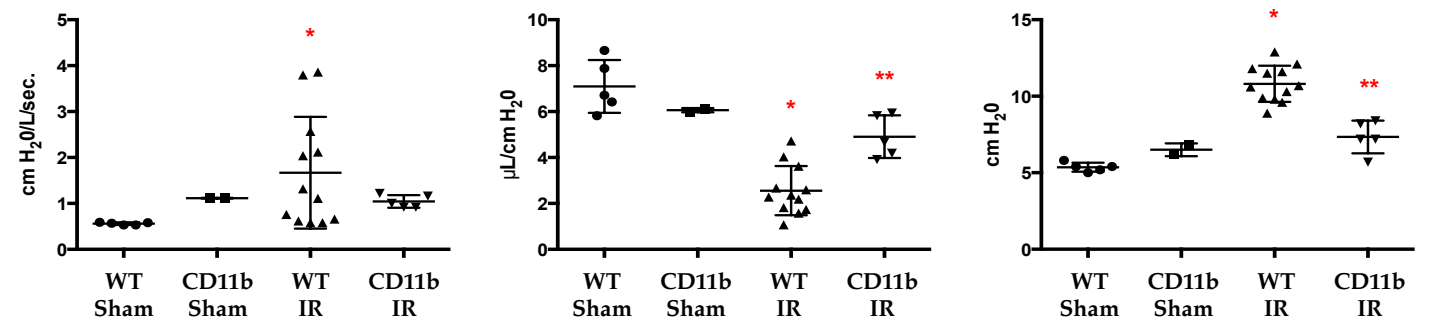

C.
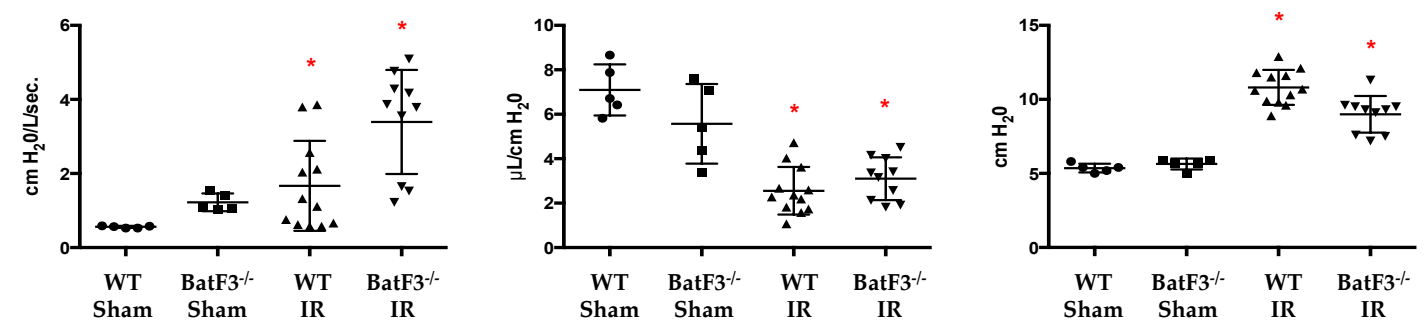

Figure 2. Functional protection from lung IR injury is achieved in CD11c-DTR (A) and CD11b-DTR (B) mice. BatF3-/- mice (C) are not functionally protected from lung IR injury. Functional protection from lung IR injury is demonstrated as decreased airway resistance, increased pulmonary compliance and decreased pulmonary artery pressure. ${ }^{*} p<0.05$ vs. WT Sham, ${ }^{* *} p<0.05$ vs. WT IR. 
Pro-inflammatory Cytokine Production is Significantly Increased Following Lung IR Injury and Decreased in CD11c-DTR Mice

Bronchoalveolar lavage samples ( $\mathrm{n}=4-8 /$ group $)$ were collected from two

independent groups of mice and cytokine analyses reported represent corroborating data.

In comparison to WT BMT IR control mice, the CD11c-DTR BMT and BatF3-/- mice

demonstrated a significant decrease in IL-12/IL-23p40 (Figure 3). IL-17 levels were not

significantly increased in WT mice following IR, limiting protective comparisons between groups. CD11b-DTR mice have significantly increased production of IL-17 following IR compared to WT IR control.

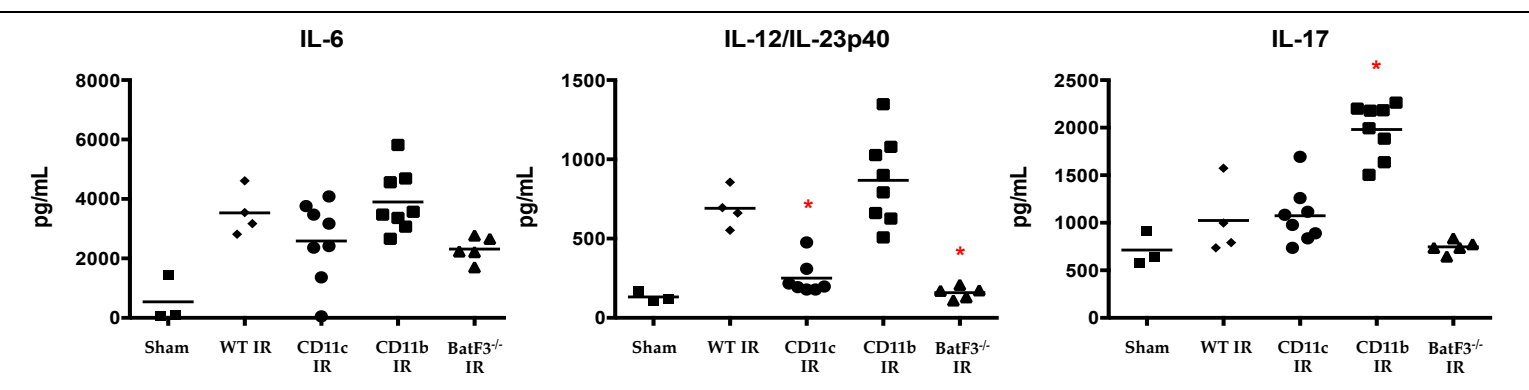

Figure 3. Pro-inflammatory cytokine production is reduced in CD11c-DTR mice following lung IR injury. IL-6 and IL-12/IL-23p40 pro-inflammatory cytokine levels are significantly decreased in CD11c-DTR mice yet not in CD11b-DTR mice following lung IR Injury. ${ }^{*} p<0.05$ vs. WT IR control.

Neutrophil Activation and Infiltration are Significantly Decreased in CD11c-DTR Mice Following Lung IR

Myeloperoxidase (MPO) concentration in bronchoalveolar lavage fluid was measured as an indicator of neutrophil infiltration. As expected, MPO was significantly elevated in WT BMT mice after IR but attenuated activation was demonstrated in both CD11c-DTR and CD11b-DTR mice (Figure 4). CD11c-DTR mice also demonstrated decreased neutrophil infiltration compared to WT BMT IR controls upon histologic evaluation. 


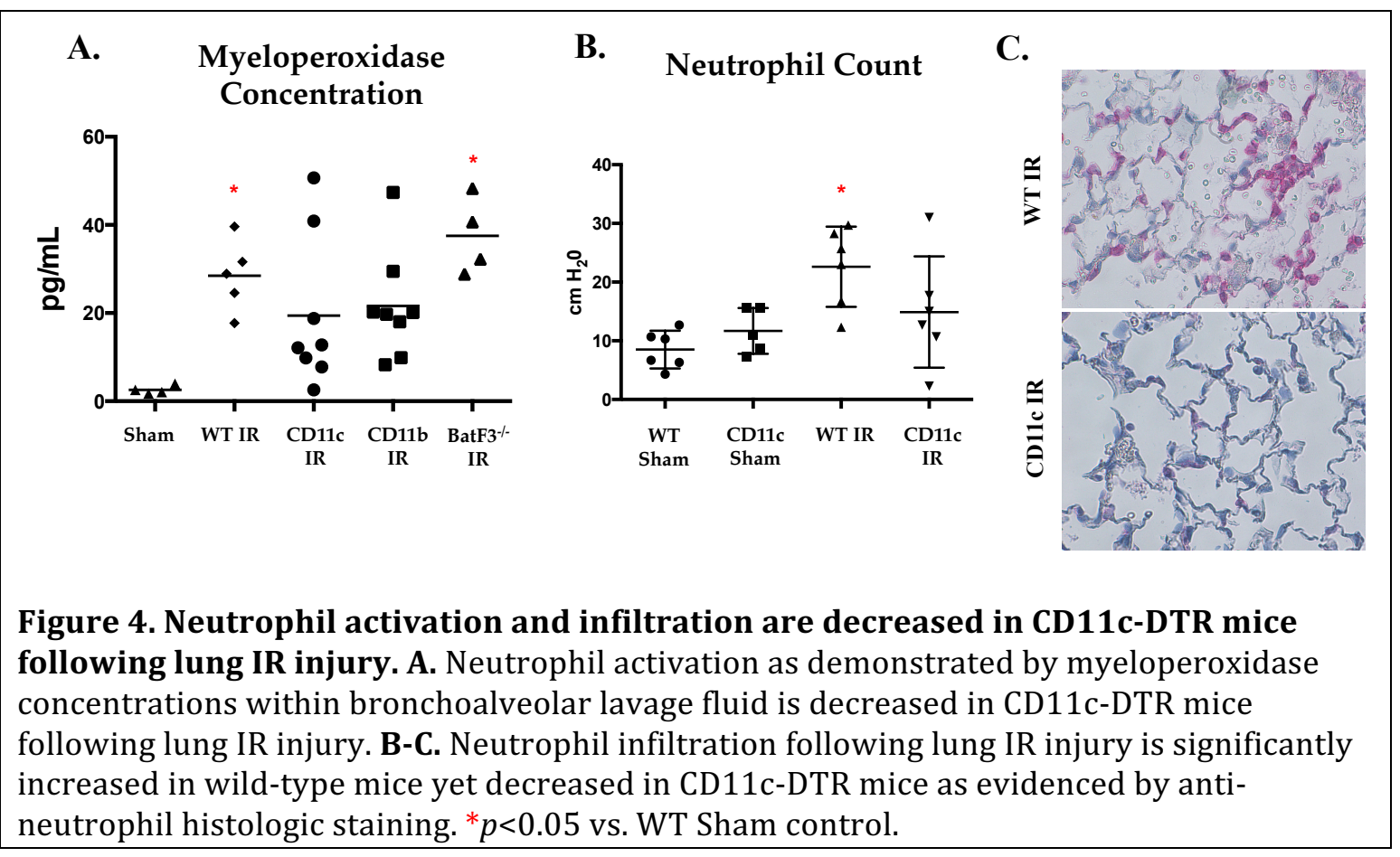

\section{IL-23p19 Gene Expression is Attenuated in CD11c-DTR Mice Following IR}

RT-PCR analysis of gene expression profiles demonstrated a significant decrease in IL-23p19 mRNA levels in the CD11c-DTR IR group in comparison to the WT BMT IR control (Ct levels: $0.4 \pm 0.04$ vs. $4.0 \pm 1.9$, respectively, $p=0.02$ )(Figure 5).

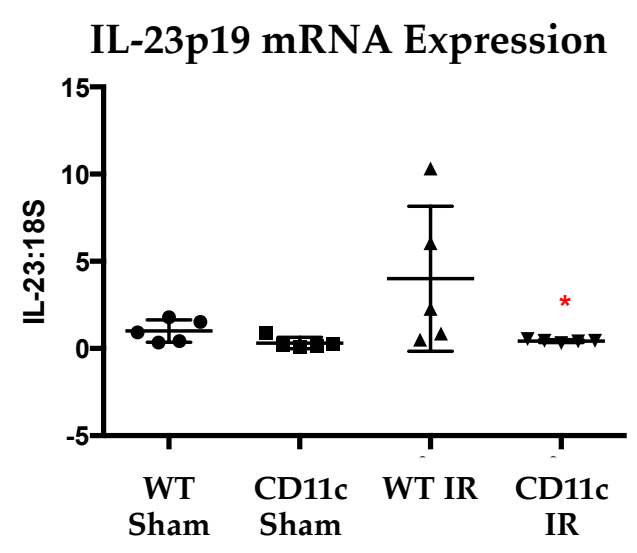

Figure 5. IL-23p19 mRNA expression is increased in WT mice and abrogated in CD11c-DTR mice following lung IR injury. Transcriptional analysis demonstrates increased gene transcription of IL-23p19 following IR injury in WT yet not CD11c-DTR mice following lung IR injury. ${ }^{*} \mathrm{p}<0.05$ vs. WT IR. 


\section{IL-23p19-/- and IL-12p35-/- Mice Have Decreased Lung Injury Following IR}

Functional evaluation of cytokine knockout mice demonstrated decreased lung injury most prominent in the IL-23p19-/- mice (Figure 6). IL-23p19-/- mice had decreased airway resistance, increased pulmonary compliance, and decreased pulmonary artery pressures compared to WT IR controls. Additionally, IL-23p19-/- mice had increased pulmonary compliance and decreased pulmonary artery pressure compared to IL-12p40-/and IL-12p35-/- mice. IL-12p35-/- mice had decreased airway resistance and pulmonary artery pressures in comparison to WT IR control. Similarly, IL-12p40-/- mice had decreased pulmonary artery pressures compared to WT IR control.

Figure 6. IL-23p19-/- mice have decreased functional lung injury when compared to WT,
IL-12p40-/-, and IL-12p35-/- groups. IL-23p19-/- mice have decreased airway resistance,
increased pulmonary compliance, and decreased pulmonary artery pressures following
lung IR injury when compared to control mice in addition to IL-12p40-/- and IL-35-/- mice.
p<0.05 vs. WT IR, $A$ p<0.05 vs. IL-12p35-/-, p<0.05 vs. IL-12p40-/-.

\section{Discussion}

The present study identified CD11c+ pulmonary macrophages as principal mediators of lung IR injury independent of pulmonary dendritic cells. Flow cytometry studies demonstrated that CD11b-DTR and CD11c-DTR transgenic mice are differentially depleted of distinct dendritic cell and macrophage populations[39]. Cytokine analysis links CD11c+ cells to the production of related pro-inflammatory cytokines IL-12 and IL-23 within the Th17 axis. Further, genetic knockout of each cytokine subunit demonstrates 
decreased functional injury across groups which was most significant within the IL-23p19/- mice. Together these findings both directly and indirectly implicated macrophagemediated IL-23p19 as an important mediator of lung IR injury, while also supporting a role for dendritic cells in IR injury pathogenesis.

Foundational to the inherent complexity of dendritic cell and macrophage identification in the lung is the shared positivity for CD11c within both dendritic cell and macrophage populations[31]. Established gating strategies within both CD11c-DTR and CD11b-DTR constructs allowed the conditional depletion of CD103+ and CD11bhi dendritic cells and CD11c+ macrophages[45]. These findings along with the established BatF3-/model for CD103+ dendritic cell depletion uniquely supported the paired and independent study of these cell populations within lung IR injury. We uniquely demonstrate that macrophages and CD103+ dendritic cells are depleted in CD11c-DTR mice and that CD11bDTR mice are depleted solely of CD11bhi dendritic cells. Prior study has demonstrated a role for CD103+ dendritic cells in the activation of invariant natural killer T cell (iNKT) cells following antigenic stimulation in infectious models[7]. Our prior study established the iNKT cell as a principal mediator of lung IR injury, supporting our study of dendritic cells and macrophages to establish efficient pharmacologic targets for IR injury modulation[3]. While macrophages have been previously implicated in IR injury pathogenesis, the present study identifies cellular markers for alveolar macrophages within a genetic knockout model both independent of and in concert with pulmonary dendritic cells which has yet to be described[6]. Infectious models of respiratory intranasal antigen administration have implicated dendritic cells independent of macrophages in iNKT cell activation, while the present study in IR injury implicates macrophages independent of dendritic cells in the mediation of pro-inflammatory pathways involving iNKT cells[11]. Together these findings 
suggest a distinction between antigen-dependent and antigen-independent responses within lung injury and subsequent iNKT cell activation.

The study of acute lung allograft rejection has identified the clustering of recipient $\mathrm{T}$ cells and lung-resident and donor-derived CD11c+ cells after engraftment[31]. This novel finding in the study of adaptive immunity importantly establishes the presence of CD11c+ and $\mathrm{T}$ cell interactions within the lung independent of secondary lymphoid organs. Additional study of innate immunity in lung transplantation has identified bone-marrow derived dendritic cell interactions with neutrophils as a contact-dependent mechanism for IL-12 production[32]. While suggesting a role for CD11c+ cells in T cell activation, neutrophil infiltration, and pro-inflammatory cytokine production, these prior studies provided indirect linkage of each yet form supporting evidence for the findings presented herein[8]. The present study demonstrates that macrophages independent of dendritic cells are principal downstream mediators of both T cell activation and neutrophil infiltration within innate immunity, elucidating cell-signaling mechanisms inherent to prior models of lung injury.

IL-23 and IL-12 were evaluated within the presented model as each has been implicated as principal mediators of CD11c+ derived pro-inflammatory cell signaling[46]. IL-23 and IL-12 are members of the same cytokine family and share expression of the p40 subunit. These related cytokines are, therefore, distinguished by the differential expression of p19 (IL-23) and p35 (IL-12) subunits, respectively[47].

The IL-23 and IL-12 axes are proposed regulators of both renal and hepatic IR injury, with CD11c+ cells serving as a principal mediator of IL-23 production in a model of renal IR injury[10,33]. Additionally, prior study has demonstrated that hypoxia-induced adenosine metabolism upregulates IL-23 receptor mRNA production in CD4(+) T cells in response to dendritic cell signaling initiated at the adenosine A2B receptor[48]. Paired with 
this finding, we have previously demonstrated a role for adenosine A2B receptor in lung IR injury and herein provide a mechanistic consideration for A2B receptor knockout mediated protection through IL-23p19 cytokine signaling[49]. Further, models in infectious disease have demonstrated that an interruption in dendritic cell IL-23 production is possible through the modulation of toll-like receptor 2 and Nod2 signaling pathways, providing signaling targets for therapeutic modulation of IL-23 pro-inflammatory responses[50]. These prior studies support our findings that IL-23 is the principal mediator of acute lung IR injury and further provide a link to established findings that invariant natural killer T-cells are principal end-effector cells of acute IR injury[51].

Two recent studies have established the iNKT cell as a key end-mediator of lung and kidney IR injury through IL-17 and IFN- $\gamma$ dependent mechanisms, respectively[3, 47]. Further, lung IR injury has been found to predispose to anti-col(V)-mediated pathology, implicating the reactive expression of IL-17 and IL-23 in col(V)-reactive lymphocytes as integral events in lung rejection pathology[13]. Clinical study has offered a linkage between acute lung injury and chronic rejection (bronchiolitis obliterans)[24]. Thus, while the present study within a model of acute lung injury offers insight to early therapeutic targets for IR injury prevention, such targets provide promising avenues for further study as mediators of short- and long-term rejection pathology within lung transplantation.

While the present study offers novel insights into lung IR injury pathogenesis, inherent and recognized limitations exist. As previously acknowledged, mouse DTR depletion models targeting CD11c and CD11b cell populations introduce the potential for depletion of unrecognized subpopulations within both macrophage and dendritic cell populations in the lung. The presented flow cytometry analysis sought to provide clarity to cell populations affected by each model utilizing established gating strategies for each closely related cell population. Preliminary data established the CD11c+ cell population as 
having increased IL-23 and IL-12 production (data not shown) yet did not provide the differentiation between macrophage and dendritic cell populations as is afforded by the presented model. While this approach provides cell-specific study, the present models limit study of cross-talk mechanisms between each in the modulation of both pro- and antiinflammatory signaling. This limitation provides a potential explanation to the variable cytokine profiles within each knockout group as prior study has demonstrated that vaccines targeting the p19 subunit result in increased IL-10 anti-inflammatory effects without any effects on IL-17 pro-inflammatory production[52]. This finding provides a potential explanation for the decreased lung IR injury within the CD11b-DTR group while having increased pro-inflammatory cytokine production, with the hypothesis that while early injury is reduced by targeted CD11b+ cell depletion, a longer temporal period of evaluation may elucidate less protection as evidenced by the cytokine profiles. Secondly, each model is dependent on reproducibility of toxin administration and inherent variability does exist in both systemic absorption and dose effect. This confounding effect was minimized by administration of toxin to WT mice and standardization of both dosing method and timing prior to IR injury. Differential depletion; however, is a potential explanation for variability demonstrated within experimental groups as each mouse studied is represented.

Additionally, while IL-23p19 levels were noted to increase in WT mice following IR, a longer period of reperfusion may demonstrate higher levels of production as has been shown in hepatic IR injury following 120 minutes of reperfusion[10]. A parallel study in renal IR injury has demonstrated increasing p19 and p40 transcripts out to 6 hours following renal IR[36]. The present study utilized a well-established time course model for lung IR injury and such extrapolations extend beyond the focus of the present study $[3,4]$. Further, histologic and gene expression analyses focused on CD11c-DTR mice as these mice demonstrated protection by both functional and pro-inflammatory cytokine production 
parameters. Evaluation of other experimental groups at this level was not pursued as neutrophil and transcriptional data are of limited importance in the absence of functional and pro-inflammatory cytokine mediated protection from lung IR injury. Such study in the future may; however, aid in the identification of cross-talk mechanisms between these cell populations and the effect of each on both cell signaling and neutrophil activation.

\section{Conclusion}

CD11c-DTR and CD11b-DTR mice afford selective conditional depletion of dendritic cell and macrophage populations within the lung. Selective depletion of CD11c+ macrophages within CD11c-DTR mice affords functional, histologic, and cytokine-mediated protection from lung IR injury independent of related dendritic cell populations.

Additionally, IL-23p19 expression is increased following lung IR injury in WT mice yet not CD11c-DTR mice, providing a link between macrophage mediated IL-23p19 production and lung IR injury pathogenesis. Further, IL-23p19 genetic knockout provides significant functional protection from lung IR injury when compared to WT mice and knockout of related cytokine subunits IL-12/IL-23p40 and IL-12p35. Together these findings support a role for macrophage and IL-23p19-targeted therapies for the prevention of lung IR injury. 


\section{Pharmacologic Targets for Lung IR Injury Prevention}

A novel sphingosine agonist, VPC-01091, is evaluated as a potential pharmacologic approach to lung IR injury prevention. Signaling mechanisms are explored at the sphingosine receptor subtype level to define implicated receptor subtypes and the effect on iNKT cell recruitment and activation. 


\section{Sphingosine-1-phosphate receptor 1 agonism attenuates lung ischemia-reperfusion injury}

Matthew L. Stone ${ }^{1}$, Ashish K. Sharma ${ }^{1}$, Yunge Zhao ${ }^{1}$, Eric J. Charles ${ }^{1}$, William F. Johnston ${ }^{1}$, Irving L. Kron ${ }^{1}$, Kevin R. Lynch ${ }^{2}$ and Victor E. Laubach ${ }^{1}$

Departments of ${ }^{1}$ Surgery and ${ }^{2}$ Pharmacology, University of Virginia, Charlottesville, VA

Stone ML et al. Am J Physiol Lung Cell Mol Physiol 2015;308(12): L1245-52. 


\section{Abstract}

Outcomes for lung transplantation are the worst of any solid organ, and ischemiareperfusion (IR) injury limits both short- and long-term outcomes. Currently no therapeutic agents are available to prevent IR injury. Sphingosine 1-phosphate (S1P) modulates immune function through binding to a set of G protein-coupled receptors (S1PR1-5). Although S1P has been shown to attenuate lung IR injury, the S1P receptor(s) responsible for protection have not been defined. The current study tests the hypothesis that protection from lung IR injury is primarily mediated through S1PR1 activation. Mice were treated with either vehicle, FTY720 (a non-selective S1P receptor agonist) or VPC01091 (a selective S1PR1 agonist and S1PR3 antagonist) prior to left lung IR. Function, vascular permeability, cytokine expression, neutrophil infiltration and myeloperoxidase levels were measured in lungs. After IR, both FTY720 and VPC01091 significantly improved lung function (reduced pulmonary artery pressure and increased pulmonary compliance) versus vehicle control. In addition, FTY720 and VPC01091 significantly reduced vascular permeability, expression of pro-inflammatory cytokines (IL-6, IL-17, IL-12/IL-23 p40, CCL2, TNF- $\alpha$ ), myeloperoxidase levels and neutrophil infiltration compared to control. No significant differences were observed between VPC01091 and FTY720 treatment groups. VPC01091 did not significantly affect elevated iNKT cell infiltration after IR, and administration of an S1PR1 antagonist reversed VPC01091-mediated protection after IR. In conclusion, VPC01091 and FTY720 provide comparable protection from lung injury and dysfunction after IR. These findings suggest that S1P-mediated protection from IR injury is mediated by S1PR1 activation, independent of S1PR3 and that selective S1PR1 agonists may provide a novel therapeutic strategy to prevent lung IR injury. 


\section{Introduction}

Outcomes for lung transplantation are the worst of any solid organ[15, 53]. Despite significant advancements over the past decade in lung transplantation, outcomes remain poor, and both short- and long-term graft survival is limited by the inherent threat of ischemia-reperfusion (IR) injury. Mechanisms involved in lung IR injury include oxidative stress, epithelial cell apoptosis, alveolar macrophage activation, $\mathrm{T}$ cell activation and neutrophil infiltration[3, 5, 6, 54-56]. Currently no therapeutic agents are available to prevent lung IR injury, and treatment strategies are limited to maintaining pulmonary mechanics and oxygenation capacity.

Sphingolipids are ubiquitous components of cell membranes, and their metabolites (e.g. ceramide, sphingosine and sphingosine 1-phosphate) are established regulators of a vast number of cellular processes[57]. Sphingosine kinase 1 and 2 phosphorylate sphingosine to generate sphingosine 1-phosphate (S1P), a biologically active lipid growth factor that binds to a family of five G-protein-coupled receptors (S1PR1-5) to regulate a spectrum of biologic functions including proliferation, cell survival, angiogenesis, extracellular matrix assembly, endothelial cell barrier integrity, and immune cell trafficking and function[58-61]. Okazaki et al. have demonstrated that S1P increases oxygenation capacity following experimental lung transplantation while decreasing pro-inflammatory cytokine production, endothelial cell apoptosis, and neutrophil numbers[62]. Other studies have shown that S1P or FTY720, a non-specific agonist for S1PR1 and S1PR3-5[63, 64], provides significant protection in various models of acute lung injury[65-67]. While these studies serve as a foundation for potential S1P receptor-targeted therapies in lung injury and transplantation, pharmacologic S1P analogs with differential receptor subtype affinities have yet to be examined in the setting of lung IR injury or transplantation. Thus, the purpose of this study was to evaluate the potential protective advantages afforded by 
VPC01091, a novel sphingosine analog that is a selective S1PR1 agonist and S1PR3 antagonist [68], on lung IR injury. In addition, we sought to concurrently evaluate FTY720 to determine the differential effects of S1PR3 targeting on lung IR injury. We approached this study with the hypothesis that selective S1PR1 agonism will attenuate lung IR injury and that S1PR3 antagonism would provide an additional protective advantage, as S1PR3 has been implicated as a biomarker of acute lung injury [69].

\section{Materials and Methods}

Study design and animals. Wild-type mice (C57BL/6, 8-12 weeks, The Jackson Laboratory, Bar Harbor, ME) were randomly assigned to six groups. All animals were treated with either vehicle or test compound via intraperitoneal injection 30 minutes prior to surgery. Two groups (sham and IR) were treated with $0.2 \mathrm{~mL}$ vehicle $(3 \%$ fatty acid-free bovine serum albumin/PBS solution, Sigma, St. Louis, MO). Two groups (sham and IR) were treated with FTY720 (2-amino-2-(4-octylphenethyl)propane-1,3-diol, $0.3 \mathrm{mg} / \mathrm{kg}$, Novartis, Basel, Switzerland), and two groups (sham and IR) were treated with VPC01091 (((1R,3S)1-amino-3-(4-octylphenyl)cyclopentyl)methanol, $1.5 \mathrm{mg} / \mathrm{kg}$, gift from Abbott Laboratories in Worcester, MA). A separate IR group entailed the co-administration of VPC01091 with VPC44116 ((R)-(3-amino-4-((3-octylphenyl)amino)-4-oxobutyl)phosphonic acid, $10 \mathrm{mg} / \mathrm{kg}$, gift from Dr. Frank Foss at the University of Texas Arlington), a selective S1PR1 antagonist [70]. Mice then underwent sham surgery or lung IR 30 minutes after intraperitoneal treatment, as this time frame has been established to achieve maximal drug effectiveness [71]. A prior time-course study has also demonstrated that FTY720-mediated barrier enhancement is maximized at 30-60 minutes following administration [72]. The present study conformed to the Guide for the Care and Use of Laboratory Animals published by the National Institute of Health and was approved by the University of Virginia Institutional Animal Care and Use Committee. 
Murine lung IR injury. An established murine model of lung IR injury was utilized as previously described by our laboratory $[3,5]$. Inhalational isoflurane anesthesia and orotracheal intubation permitted mechanical ventilation at 120 strokes/min with room air. Heparin was administered via the right external jugular vein $(20 \mathrm{U} / \mathrm{kg})$, and the left pulmonary hilum was exposed through an anterolateral thoracotomy at the third intercostal space. A 6-0 Prolene suture was passed around the left pulmonary hilum, and the two suture ends were passed through PE-60 tubing to permit hilar occlusion via tightening of suture and surgical clip application. Analgesia was administered (buprenorphine, $0.2 \mathrm{mg} / \mathrm{kg}$ ) by intraperitoneal injection, and animals were returned to their cage during the 1 hour of left-lung ischemia. Mice then underwent repeat anesthesia and intubation, and the hilar occlusion was released to begin reperfusion. Animals were then returned to their cages whereupon reperfusion was continued for 2 hours prior to functional evaluation, bronchoalveolar lavage and histologic analysis. Sham groups were identical to IR groups except that the left hilum was not occluded.

Pulmonary function. Pulmonary function at the end of reperfusion was measured using an isolated, buffer-perfused lung apparatus (Hugo Sachs Elektronik, MarchHuggstetten, Germany) as previously described [4]. Mice were anesthetized and maintained on intra-tracheal ventilation (tidal volume $=7 \mu \mathrm{L} / \mathrm{g}$ body weight, rate $=100$ strokes $/ \mathrm{min}$, positive end-expiratory pressure $=2 \mathrm{cmH}_{2} \mathrm{O}$ ) prior to exsanguination by inferior vena caval transection. The pulmonary artery was cannulated and a left ventriculotomy permitted perfusate drainage. Lungs were perfused at a flow rate of $60 \mu \mathrm{L} / \mathrm{g}$ body weight/min with Krebs-Henseleit buffer. Following a 5-minute period of equilibration, functional data (pulmonary artery pressure and pulmonary compliance) were recorded using PULMODYN data acquisition software (Hugo Sachs Elektronik) over an additional 5 minutes. 
Bronchoalveolar lavage (BAL). Following measurement of lung function, the left lung was isolated via ligation of the right pulmonary hilum with a surgical clip. An anterior tracheotomy was then performed and permitted intra-tracheal placement of a 20-Gauge angiocatheter. A circumferential suture was secured around the trachea to limit pericatheter drainage. Two consecutive aspirates of $0.4 \mathrm{~mL}$ of $0.9 \%$ sodium chloride were then performed through the intra-tracheal cannula. Left lung BAL fluid was immediately centrifuged at $4^{\circ} \mathrm{C}(1,500 \mathrm{rpm}$ for $6 \mathrm{~min})$, and supernatant was stored at $-80^{\circ} \mathrm{C}$.

Cytokine and myeloperoxidase (MPO) measurements. As previously described [73], cytokines were quantified in BAL fluid using a multiplex ELISA cytokine panel (BioRad Laboratories, Hercules, CA), and MPO concentration in BAL fluid was measured by ELISA (Hycult Biotech, Uden, Netherlands).

Immunohistochemistry and neutrophil counting. Using separate groups of animals, lungs were inflation-fixed at $10 \mathrm{cmH}_{2} \mathrm{O}$ with formalin at $4^{\circ} \mathrm{C}$ for 24 hours prior to placement in $70 \%$ ethanol for paraffin embedding. Lung sections were immunostained for neutrophils using the Vectastain ABC kit (Vector Laboratories, Burlingame, CA) as previously described [4]. Rat anti-mouse neutrophil antibody (AbD Serotec, Raleigh, NC) and alkaline phosphatase-conjugated anti-rat IgG (Sigma, St. Louis, MO) secondary antibody were utilized. The signals were detected using Fast-Red (Sigma, St. Louis, MO). The negative control utilized purified normal rat IgG (eBioscience Inc., San Diego, CA). Neutrophil counting was performed by a blinded investigator. Three semi-standardized fields per lung were counted at 20x magnification, and the mean of these three values established the final neutrophil count per high-powered field for each mouse.

Pulmonary vascular permeability. Vascular permeability in lungs was estimated using the Evans blue dye extravasation technique, which is an index of change in protein permeability, as previously described [74]. Using separate groups of animals ( $n=5 /$ group), 
Evans blue (20 mg/kg, Sigma, St. Louis, MO) was injected intravenously via the tail vein 30 min before euthanasia. The pulmonary vasculature was then perfused for 10 min with PBS to remove intravascular dye. Lungs were then homogenized in PBS to extract the Evans blue and centrifuged. The absorption of Evans blue was measured in the supernatant at $620 \mathrm{~nm}$ and corrected for the presence of heme pigments: $\mathrm{A} 620_{\text {corrected }}=\mathrm{A} 620-(1.426 \times \mathrm{A} 740+$ 0.030). The concentration of Evans blue was determined according to a standard curve and expressed as micrograms/gram ( $\mu \mathrm{g} / \mathrm{g})$ wet lung weight.

Flow cytometry. Quantification of invariant natural killer T (iNKT) cells via flow cytometry was performed as previously described [3]. Left lungs from mice were minced and incubated for $15 \mathrm{~min}$ at $37^{\circ} \mathrm{C}$ with collagenase type $1 \mathrm{~A}$ (Sigma-Aldrich, St. Louis, MO) in Dulbecco's PBS with $0.5 \%$ BSA and 2mM EDTA. The lung tissue suspension was then passed through a $40-\mu \mathrm{m}$ cell strainer (BD Falcon) and centrifuged at $1000 \mathrm{rpm}$ for $10 \mathrm{~min}$. After treatment with RBC lysis buffer, the cell pellet was washed and resuspended in FACS buffer (0.1\% BSA, $0.01 \%$ sodium azide in PBS). Cells were stained with 7-aminoactinomycin (7AAD; Invitrogen, Frederick, MD), PerCP-Cy5.5-labelled CD45 (eBioscience, San Diego, CA), PE-labelled CD1d tetramer loaded with PBS57 (1:500), an analog of $\alpha$-galactosylceramide ( $\alpha$ GalCer) (NIH Tetramer Facility, Emory University, Atlanta, GA) and FITC-labelled CD4 (eBioscience). For cell counting, $100 \mu \mathrm{l}$ of cell suspension was mixed thoroughly with $100 \mu \mathrm{l}$ of Caltag Counting Beads (Life Technologies, Frederick, MD) before acquisition by FACS. At least 1000 bead events were acquired to ensure the accuracy of the assay. The absolute number of any gated cell population was calculated as follows: CD45+ cell absolute count (per lung $)=($ events of CD45+ cells counted/total number of beads counted $(A+B) X$ input bead number)/lung. The total number of iNKT cells (per lung) was calculated by multiplying the CD45+ cell number and the percentage of the CD4+CD1d tetramer+ subset. 
For example, the CD4+CD1d tetramer+ cell number (per lung $)=($ total $C D 45+$ cell number $)$ $\mathrm{X}$ (percent of CD4+CD1d tetramer+ cells gated on the CD45+ cell population).

Statistics. Statistical analyses were performed using a one-way analysis of variance (ANOVA) with a post-hoc Tukey's multiple comparisons correction. A $p$ value of less than 0.05 represented statistical significance. Results are presented as the mean $\pm \mathrm{SEM}$.

\section{Results}

\section{Pulmonary function after IR is improved by VPC01091 and FTY720 treatment.}

To determine if S1PR1 agonism improves lung function after IR, mice were treated with VPC01091 or FTY720 prior to ischemia or sham surgery. Vehicle-treated mice demonstrated significant lung dysfunction following IR as shown by increased pulmonary artery pressure and decreased pulmonary compliance (Figure 7). Compared to vehicle, both FTY720 and VPC01091 significantly decreased pulmonary artery pressure and increased pulmonary compliance after IR. No significant differences were observed between sham groups treated with vehicle, VPC01091 or FTY720 (Figure 7). These results demonstrate that selective agonism of S1PR1 by VPC01091 improves lung function after IR to the same level as FTY720. 

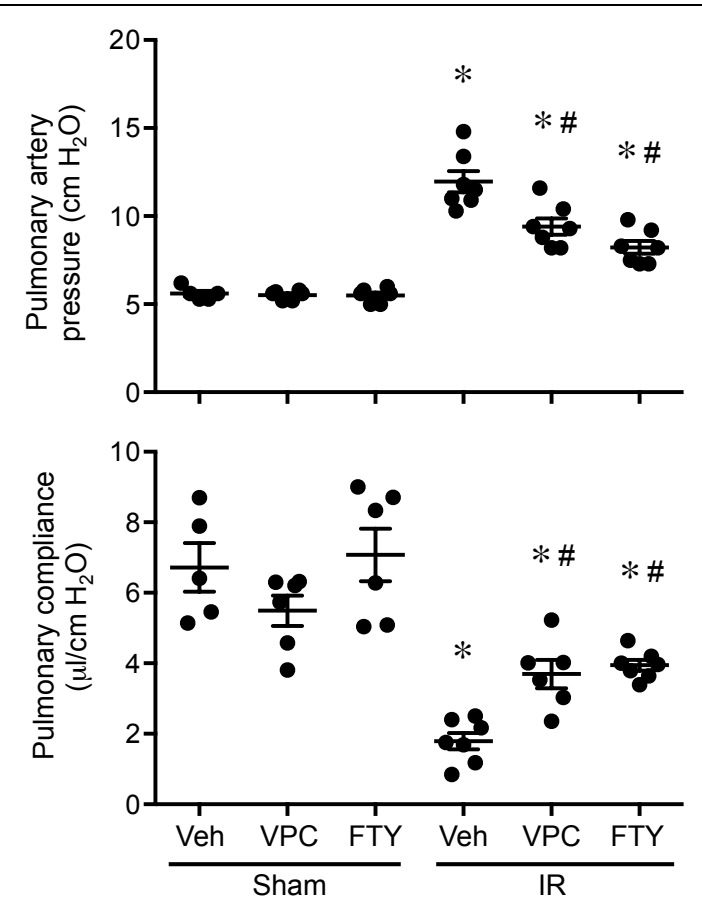

Figure 7. VPC01091 and FTY720 treatment improves lung function after ischemiareperfusion (IR). Mice were pre-treated with vehicle (Veh), VPC01091 (VPC) or FTY720 (FTY) 30 minutes prior to sham surgery or ischemia. Pulmonary artery pressure and pulmonary compliance were measured after two hours of reperfusion. Results are presented as mean \pm SEM. ${ }^{*} \mathrm{p}<0.05$ vs. vehicle sham, ${ }^{*} \mathrm{p}<0.05$ vs. vehicle IR, $\mathrm{n}=5-7 /$ group.

VPC01091 and FTY720 reduces pulmonary vascular permeability after IR. To

investigate the extent to which VPC01091 or FTY720 affect vascular permeability after IR, pulmonary vascular leak was assessed using the Evan blue dye extravasation technique. IR significantly increased vascular permeability, which was significantly attenuated by VPC01091 or FTY720 (Figure 8). These results demonstrate that FTY720-mediated attenuation of vascular permeability after IR can be reproduced by selective S1PR1 agonism by VPC01091. 


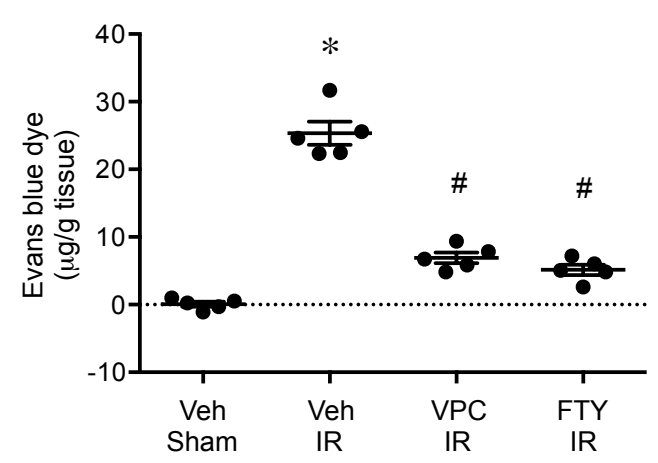

Figure 8. VPC01091 and FTY720 treatment attenuates pulmonary vascular permeability after ischemia-reperfusion (IR). Mice were pre-treated with vehicle (Veh), VPC01091 (VPC) or FTY720 (FTY) 30 minutes prior to sham surgery or ischemia. Vascular permeability was assessed after two hours of reperfusion by measuring Evans blue dye content in the lung ( $\mathrm{g} / \mathrm{g}$ lung tissue) as described in the methods. Results are presented as mean \pm SEM. ${ }^{*} \mathrm{p}<0.05$ vs. vehicle sham, ${ }^{*} \mathrm{p}<0.05$ vs. vehicle IR, $\mathrm{n}=5$ /group.

\section{VPC01091 and FTY720 attenuates pro-inflammatory cytokine production}

following IR. Pulmonary inflammation after IR was assessed by measuring levels of proinflammatory cytokines in BAL fluid. IR significantly increased expression of IL-6, IL-17, IL12 p70, IL-12/IL-23 p40, CCL2 and TNF- $\alpha$ in vehicle treated animals. VPC01091 treatment resulted in significantly decreased levels of IL-6, IL-17, IL-12p70, IL-12/IL-23p40, CCL2 and TNF- $\alpha$ following IR versus vehicle-treatment. Similarly, FTY treatment significantly decreased levels of IL-6, IL-17, IL-12p70, IL-12/IL-23p40, CCL2 and TNF- $\alpha$. Cytokine levels were similar among sham animals treated with vehicle, FTY720 or VPC01091 (Figure 9). These results demonstrate that FTY720 and VPC01091 have similar inhibitory affects on pro-inflammatory cytokine expression after IR. 


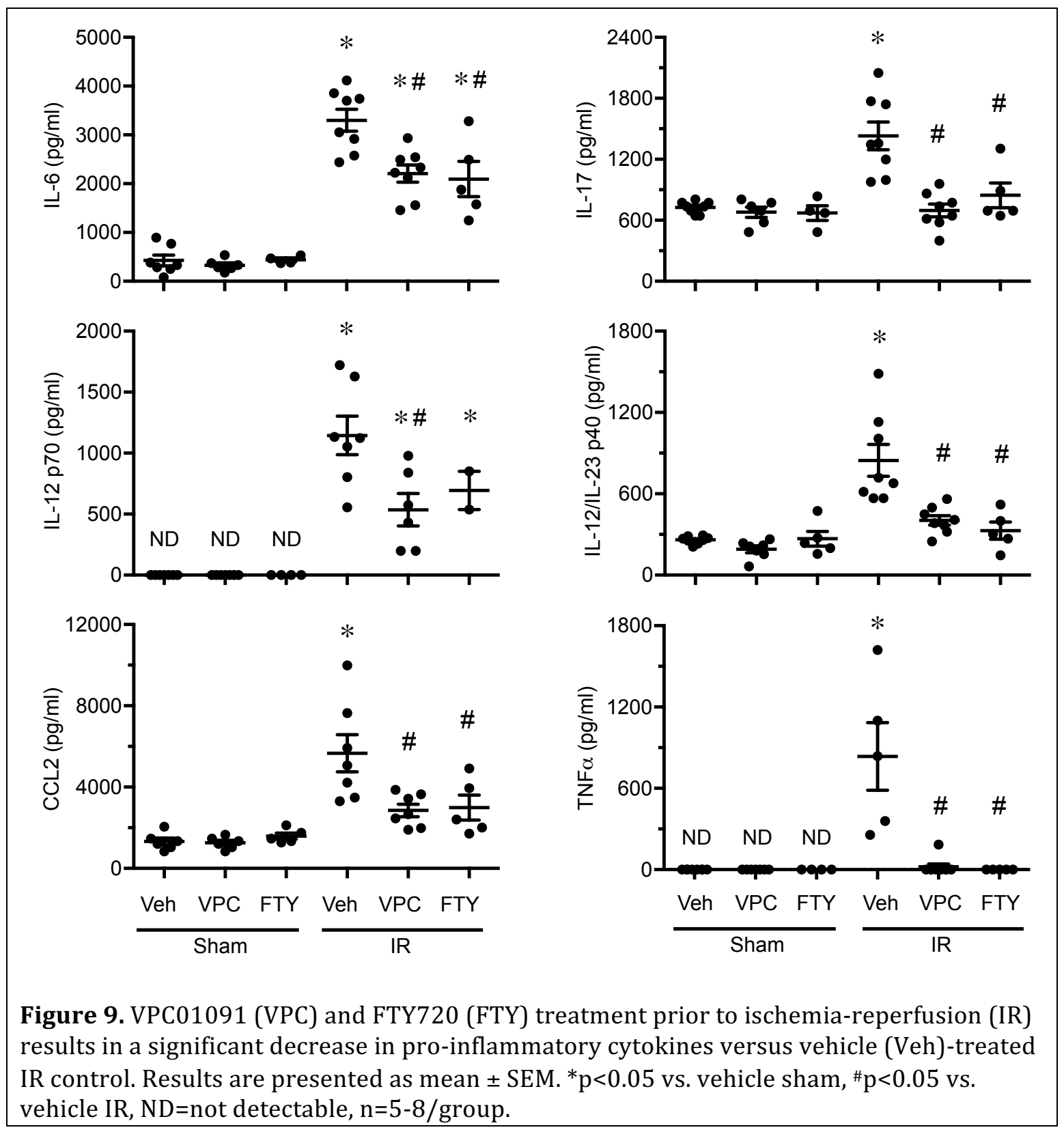

Neutrophil activation and infiltration after IR is decreased by VPC01091 and FTY720

treatment. Neutrophil infiltration is a hallmark of lung inflammation after IR, and thus neutrophil numbers and activation status were assessed in lungs. Immunostaining of lung sections demonstrated that elevated neutrophil infiltration after IR was significantly attenuated by both VPC01091 and FTY720 treatments (Figure 10A-B). There were no significant differences in neutrophil numbers between vehicle, VPC01091 and FTY720 sham 
treatment groups (data not shown). The concentration of MPO, a peroxidase enzyme abundantly present in neutrophil granulocytes and released upon activation, in BAL fluid was significantly increased after IR versus sham in vehicle-treated animals (Figure 10C). Both VPC01091 and FTY720 treatments resulted in significantly decreased MPO concentration versus vehicle-treated IR. There were no significant differences in neutrophil counts or MPO levels between sham animals treated with vehicle, VPC01091 or FTY720 (data not shown). These data suggest that FTY720-mediated affects on neutrophil infiltration and activation after IR are reproduced by selective S1PR1 agonism by VPC01091. 


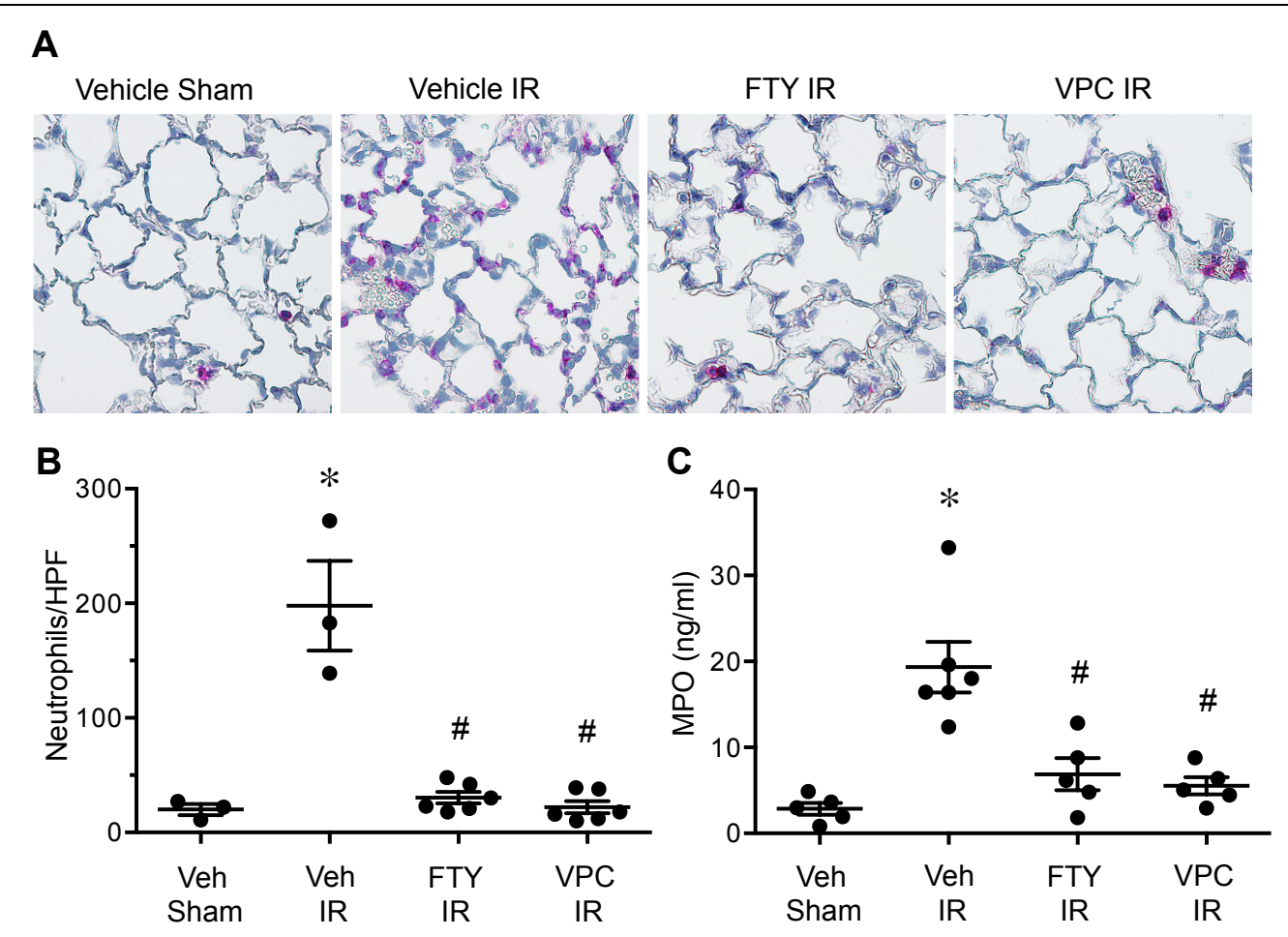

Figure 10. Neutrophil infiltration and activation after ischemia-reperfusion (IR) is significantly decreased by VPC01091 (VPC) or FTY720 (FTY) treatment compared to vehicle (Veh)-treated mice. A) Representative immunostaining of neutrophils (pink staining) within indicated groups. 40x magnification. B) Quantification of neutrophils per high-powered field (HPF) for each group. C) Myeloperoxidase (MPO) levels in bronchoalveolar lavage fluid after IR were significantly reduced by VPC and FTY treatment versus vehicle treatment. No significant differences in neutrophil counts or MPO levels between vehicle-, VPC01091- and FTY720-treatment of sham animals were observed (data not shown). Results are presented as mean \pm SEM. ${ }^{*} \mathrm{p}<0.05$ vs. vehicle sham; ${ }^{*} \mathrm{p}<0.05$ vs. vehicle IR, $n=3-6 /$ group.

\section{S1PR1 agonism is essential for VPC01091-mediated attenuation of lung IR}

injury. To determine if the S1PR3 antagonist functionality of VPC01091 potentially contributes to VPC01091-mediated protection after IR, an S1PR1 antagonist, VPC44116, was co-administered with VPC01091. Co-administration of VPC01091 and VPC44116 resulted in a reversal of protection from lung dysfunction after IR (Figure 11). VPC01091/VPC44116 combined treatment significantly reversed the VPC01091-mediated decrease in pulmonary artery pressure after IR. Similarly, VPC01091/VPC44116 combined 
treatment also reversed VPC01091-mediated improvement in pulmonary compliance, although this did not reach significance. These results confirm S1PR1 as the principal protective mediator of lung IR injury by S1P analogs such as VPC01091.

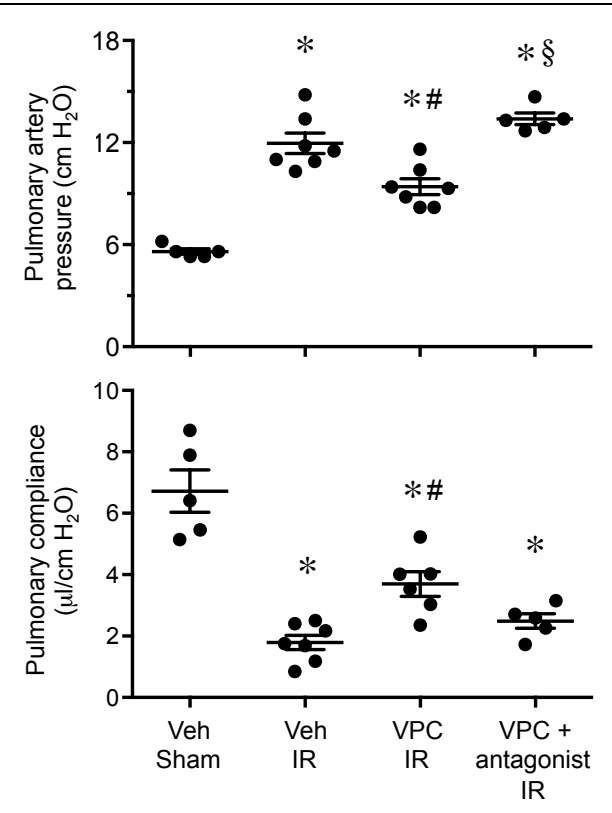

Figure 11. VPC01091 (VPC)-mediated functional protection after IR is reversed with coadministration of an S1PR1 antagonist (VPC44116). The VPC + antagonist group is shown compared to relevant groups shown in Figure 1. Results are presented as mean \pm SEM. ${ }^{*} \mathrm{p}<0.05$ vs. vehicle sham; ${ }^{*} \mathrm{p}<0.05$ vs. vehicle IR, $\S_{\mathrm{p}}<0.05$ vs. VPC IR, $\mathrm{n}=5-7 /$ group. 
VPC01091 does not affect infiltration of iNKT cells after lung IR. To identify iNKT

cells, a PE-labeled CD1d tetramer loaded with an analog of $\alpha$ GalCer was utilized as

previously described [3]. The total number of iNKT cells (CD4+ CD1d tetramer+) was

increased over 4 -fold in the left lung after IR $(16,977 \pm 3,357$ iNKT cells) compared to sham

$(71,706 \pm 8,086$ iNKT cells) in vehicle-treated mice (Figure 12). Treatment with VPC01091

did not significantly affect iNKT cell numbers after IR $(90,357 \pm 10,429$ iNKT cells) versus

vehicle (Figure 12).

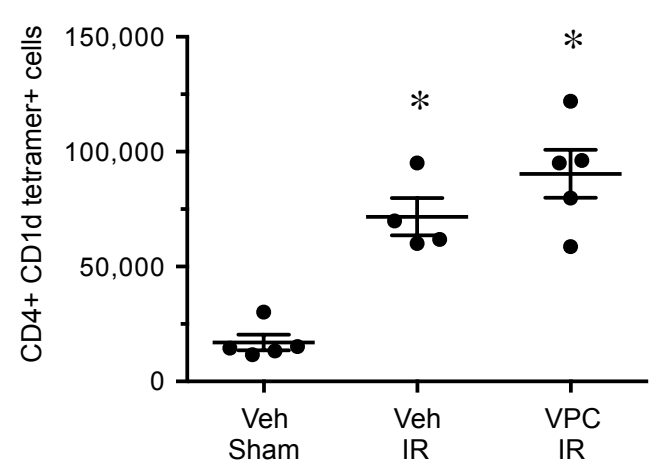

Figure 12. VPC01091 does not affect iNKT cell infiltration after ischemia-reperfusion (IR). Total iNKT cell numbers (CD4+ CD1d tetramer+ cells) were counted in left lungs by flow cytometry as described in the methods. Mice pre-treated with vehicle (Veh) or VPC01091 (VPC) demonstrated similar and significant elevations in iNKT cell numbers after IR versus sham. Results are presented as mean \pm SEM. ${ }^{*} \mathrm{p}<0.05$ vs. vehicle sham, $\mathrm{n}=4-5$ /group.

\section{Discussion}

Use of pharmacologic S1P receptor-targeted drugs have demonstrated promise in the regulation of immune-mediated disease through the inhibition of lymphocyte egress from lymphoid organs [75]. This strategy has been adopted to attenuate acute injury and graft rejection in kidney and liver transplantation with a proposed protective mechanism through S1PR1 binding $[71,76,77]$. Several studies have supported the application of S1P and FTY720 in the reduction of injury in experimental lung transplantation models[62, 78]. While these studies support a protective role for S1P and S1P analogues in IR injury, further understanding of the role of specific S1P receptor subtypes in lung IR injury is needed. 
S1PR3 has been linked to decreased epithelial integrity within the lung in addition to promoting airway hyper-reactivity, systemic hypertension, coronary artery vasoconstriction, and pro-fibrotic responses within the lung[79-84]. Additionally, the nitrated form of S1PR3 is increased in the plasma of mice and humans with sepsis-induced acute lung injury, and reduced S1PR3 expression is associated with an attenuation of increased vascular permeability during acute lung injury[69]. Thus, the design of agents with differential activities at S1P receptor subtypes may help provide optimal protection from lung IR injury with reduced negative side effects.

With this understanding, the present study evaluated the efficacy of a novel sphingosine analogue, VPC01091, that serves as a selective S1PR1 agonist and S1PR3 antagonist[68]. Comparison of VPC01091 to the non-selective FTY720 agonist demonstrated an equivalent level of protection, suggesting that that S1P- and FTY720mediated protection is achieved primarily through S1PR1 activation. While FTY720 also acts on S1P4 and S1P5 receptors, S1P5 is exclusively expressed in the brain and skin[85]. The biologic activities of S1P4 are not well established; however, a recent study has demonstrated that S1P4 deficiency results in impaired dendritic cell migration, cytokine secretion and Th-17 cell differentiation[86]. This supports a potential mechanistic role for S1P4 in IR injury pathogenesis[32, 61]. The present data demonstrates that antagonism at S1PR3 does not result in altered therapeutic efficacy of VPC01091. While superiority of VPC01091 over FTY720 in protection was not demonstrated in the present study, the use of VPC01091 may be advantageous to avoid potential long-term effects of S1PR3 receptor agonism by S1P, FTY720 or similar non-specific agonists.

Important to the advancement of pharmacologic S1P receptor agonist therapy is the timing of delivery, as S1P receptor expression at the cell membrane can vary according to the cell activation state[87]. Graeler et al. demonstrated that activation of CD4+ T cells 
results in decreased S1P receptor expression, suppressing the potentially beneficial effects of S1P administration after onset of injury[88]. However, pretreatment prior to T cell activation results in a significant inhibition of chemokine-directed migration. These prior findings provided rationale for the use a pre-treatment strategy in the present study and support the translation of this therapy to the treatment of the donor lung or transplant recipient prior to transplantation.

Debate persists regarding the mechanisms of protection afforded by S1P or FTY720 administration, supporting our application of VPC01091 as a novel agent for prevention of lung IR injury. FTY720 has been demonstrated to have more potent effects than S1P on immune cell trafficking and recruitment to secondary lymphoid organs, as it is not metabolized as efficiently as the natural ligand[60]. These findings introduce potential limitations to S1P delivery for prevention of IR injury, supporting the design and utilization of selective S1P receptor agonists that are optimized for maximal biologic effect. FTY720 treatment was found to downregulate S1PR1 expression, creating a temporary pharmacologic S1PR1-null state in lymphocytes[87]. This strategy has been adopted in models of renal IR injury, with antagonist studies suggesting the mechanism of activity to be at S1PR1[71]. The co-administration of VPC01091 with a selective S1PR1 antagonist (VPC44116) in the present study supports a similar mechanism of protection from lung IR injury through S1PR1 agonism.

A multitude of studies suggest a central role for S1P signaling in the maintenance of endothelial barrier function[89]. S1P and FTY720 have been suggested to sustain the endothelial cell barrier during a state of inflammation through S1PR1 activation and induction of hepatocyte growth factor, acting through a $\mathrm{G}_{\mathrm{i}}$-coupled receptor, tyrosine kinases and lipid rafts[90-92]. Parallel studies with S1P and FTY720 in the setting of reduced S1PR1 expression (via siRNA) have demonstrated an absence of effect on 
pulmonary endothelial barrier enhancement, suggesting that the protective effects are dependent on the S1PR1[72]. While these mechanisms of protective actions remain unclear, the present study importantly demonstrates that both FTY720 and VPC01091 provide similar protection from lung IR injury including potent preservation of endothelial barrier function. The reduction in vascular permeability by FTY720 and VPC01091 after IR (Figure 2) could, however, result from direct effects upon endothelial cells, indirect effects of reduced proinflammatory cytokines/chemokines or both. Further, while validating prior studies that support the efficacy of FTY720 in attenuation of IR injury, our utilization of VPC01091 demonstrates that antagonistic activity at the S1PR3 receptor may not limit therapeutic potential and suggest VPC01091 as a more strategic therapeutic approach for prevention of lung IR injury through S1PR1 agonism[78].

Prior study has demonstrated that S1P analogues reduce inflammation through the negative regulation of IL-12p70 following LPS administration[93]. Further, S1P is decreased in patients with cystic fibrosis, and supplementation has demonstrated the potential to rescue MHC-II and CD40 expression on lung dendritic cells[94]. These data are supported by our finding that protection from lung IR injury by FTY720 and VPC01091 was associated with decreased IL-12 (p70) expression as well as IL-12/IL-23 (p40) expression (p40 is a subunit of both IL-12 and IL-23), suggesting mechanistic effects on the dendritic cell-T cell axis during lung IR injury. The reduction in IL-12/IL-23 by FTY720 and VPC01091 likely contributed to the observed decrease in IL-17 production after IR, which confirms our previous results that demonstrate a principal role for IL-17 production by iNKT cells in lung IR injury [3]. Thus, it is possible that S1PR1 agonism attenuates lung IR injury, at least in part, by dampening the IL-23/IL-17 axis. This becomes more relevant because we observed that VPC01091 did not affect iNKT cell trafficking into the lung after IR (Figure 6), which supports findings by Hwang et al. showing that S1PR1 agonism affects NKT cells largely by 
inhibiting cytokine production rather than affecting migration[95]. Thus, results of the present study suggest that S1PR1 agonism potently attenuates lung IR injury by modulating both endothelial barrier function and iNKT cell activation.

While the present study provides important insights into the potential for S1P receptor-targeted therapies in prevention of lung IR injury, inherent limitations exist. First, the lung IR injury model entails warm ischemia and reperfusion of the lung but does not involve transplantation. While this model has been validated by prior study and is an accepted model for mechanistic studies in IR injury, immunoregulatory effects of these agents on donor-recipient cell interactions cannot be concluded from the present study. Second, the present study involves a 2-hour period of reperfusion and thus focuses on acute IR injury. While acute injury and chronic rejection pathologies have causal and associative linkage in lung transplantation, no conclusions regarding the effects of these compounds on chronic graft function can be made from the present study. Importantly, however, we predict that VPC01091, through prevention of IR injury, would benefit long-term immunoregulation and graft function. Thus it is possible that S1PR1 agonism and paired S1PR3 antagonism may provide optimal S1P receptor-targeted prevention of rejection pathology after lung transplant while limiting the pro-fibrotic activity that has been associated with S1PR3 receptor activation[58]. This hypothesis is based on a prior study in a bleomycin model of lung injury that demonstrated an exacerbation of lung injury with diffuse alveolar damage and more significant hyaline membrane deposition with repeat FTY720 administration[96].

In conclusion, the present study demonstrates that $\mathrm{S} 1 \mathrm{P}$ analogues provide a promising modality for the prevention of lung IR injury. The finding that VPC01091 affords equal protection to FTY720 in lung IR injury demonstrates that the protective mechanisms, such as preservation of endothelial barrier function or modulation of iNKT cell activation, 
are primarily dependent on S1PR1 agonism. Use of VPC01091 may, therefore, be a more effective approach to S1P receptor-targeted therapy, as it avoids the limited potency of S1P alone while also avoiding potential deleterious effects of S1P3-mediated pro-fibrotic processes after lung injury. 


\section{Ex Vivo Lung Perfusion (EVLP) as a Strategy for Donor Lung Assessment and Rehabilitation}

Ex vivo lung perfusion (EVLP) is evaluated as a strategy for donor lung rehabilitation following donation after cardiac death (DCD). A novel murine model for DCD lung rehabilitation is developed to evaluate the utilization of an adenosine $2 \mathrm{~A}$ receptor (A2AR) agonist in donor lung allograft treatment. A pre-clinical porcine model is utilized to further evaluate this strategy and to define the optimal timing for EVLP in donor lung rehabilitation.

These studies provide the foundation for current clinical trials in human lung transplantation and donor lung rehabilitation. 
Ex vivo lung perfusion (EVLP) with adenosine $2 \mathrm{~A}$ receptor agonist enhances rehabilitation of murine non-heart-beating donor lungs

Mathew L. Stone, Ashish K. Sharma, Valeria. R. Mas, Ricardo C. Gehrau, Daniel P. Mulloy, Yunge Zhao, Christine L. Lau, Irving L. Kron and Victor E. Laubach

Department of Surgery, University of Virginia, Charlottesville, VA

Stone ML et al. Transplantation 99(12):2494-503, 2015. 


\section{Abstract}

Background: Ex vivo lung perfusion (EVLP) enables assessment and rehabilitation of marginal donor lungs prior to transplantation. We previously demonstrated that adenosine $A 2 A$ receptor $\left(A_{2 A} R\right)$ agonism attenuates lung ischemia-reperfusion injury. The current study utilizes a novel murine EVLP model to test the hypothesis that $A_{2 A} R$ agonist enhances EVLP-mediated rehabilitation of donation after circulatory death (DCD) lungs.

Methods: Mice underwent euthanasia and $60 \mathrm{~min}$ warm ischemia, and lungs were flushed with Perfadex and underwent cold static preservation (CSP, $60 \mathrm{~min}$ ). Three groups were studied: no EVLP (CSP), EVLP with Steen solution for 60 min (EVLP), and EVLP with Steen solution supplemented with ATL1223, a selective $A_{2 A} R$ agonist (EVLP+ATL1223). Lung function, wet/dry weight, cytokines and neutrophil numbers were measured. Microarrays were performed using the Affymetrix GeneChip Mouse Genome 430A 2.0 Array. Results: EVLP significantly improved lung function versus CSP, which was further, significantly improved by EVLP+ATL1223. Lung edema, cytokines, and neutrophil counts were reduced after EVLP and further, significantly reduced after EVLP+ATL1223. Gene array analysis revealed differential expression of 1,594 genes after EVLP, which comprise canonical pathways involved in inflammation and innate immunity including IL-1, IL-8, IL-6 and IL-17 signaling. Several pathways were uniquely regulated by EVLP+ATL1223 including the downregulation of genes involved in IL-1 signaling such as ADCY9, ECSIT, IRAK1, MAPK12 and TOLLIP.

Conclusion: EVLP modulates pro-inflammatory genes and reduces pulmonary dysfunction, edema and inflammation in DCD lungs, which are further reduced by $A_{2 A} R$ agonism. This murine EVLP model provides a novel platform to study rehabilitative mechanisms of DCD lungs. 


\section{Introduction}

Lung transplantation provides the only curative option for many patients with endstage pulmonary disease, yet the success of this treatment is significantly limited by donor organ shortage and the inherent threat of ischemia-reperfusion (IR) injury that leads to primary graft dysfunction (PGD). Advancements over the past decade have decreased incidences of PGD from 30\% to 5-15\% while also expanding the number of donor lungs suitable for transplantation[1, 21, 23, 97-100]. A principal focus for continued progress in lung transplantation is the rehabilitation and utilization of both controlled (Maastricht category III and IV) and uncontrolled (Maastricht category I and II) non-heart-beating donor (NHBD) lungs that have traditionally carried a higher risk of PGD and mortality[101, 102].

Ex vivo lung perfusion (EVLP) is a novel technique that provides normothermic perfusion and mechanical ventilation to donor lungs, allowing functional assessment during the preservation period[103]. EVLP has been utilized as an effective strategy for the assessment of marginal, high-risk donor lungs and has demonstrated potential for expansion of the limited donor organ pool through the rehabilitation of DCD lungs[104107]. While current advancements in EVLP-mediated rehabilitation merit optimism concerning the future utilization of DCD lungs, two principal benefits to this approach that have not been explored well are the delivery of therapeutic, anti-inflammatory agents to the injured lung and the identification of molecular markers that may serve as predictors of PGD after transplantation.

We have previously demonstrated that adenosine $2 \mathrm{~A}$ receptor $\left(\mathrm{A}_{2 \mathrm{~A}} \mathrm{R}\right)$ agonism is a well-defined approach for the amelioration of lung IR injury after transplant [99, 108-110] largely through activation of $A_{2 A} R s$ on neutrophils and CD4+ T cells[100]. We have also shown that EVLP-mediated delivery of $A_{2 A} R$ agonist is a potentially effective strategy for the 
rehabilitation of Maastricht category I DCD lungs[105]. Thus, the purpose of the current study was twofold: 1) to utilize a novel murine EVLP model to test the hypothesis that administration of a selective $A_{2 A} R$ agonist during EVLP provides a superior strategy to EVLP with Steen solution alone for the rehabilitation of DCD lungs and 2) to assess the gene expression profile in NHBD lungs after EVLP.

\section{Materials and Methods}

\section{Animals}

Wild-type C57BL/6 mice (The Jackson Laboratory, Bar Harbor, ME) of 9-12 weeks of age were utilized. The Institutional Animal Care and Use Committee (IACUC) of the University of Virginia reviewed and approved this study, and standard animal care was conducted in accordance with the "Guide for Care and Use of Laboratory Animals" (National Institutes of Health publication no. 85-23, revised 1985).

\section{Murine Lung DCD Procedure}

Mice were anesthetized by isoflurane inhalation and euthanized by cervical dislocation followed by a 60-minute period of "no-touch" warm ischemia. Mice then underwent extended median sternotomy and midline cervical exposure followed by orotracheal or tracheostomy-mediated intubation for the initiation of mechanical ventilation at 120 strokes/minute with room air. The left atrial appendage was ligated prior to infusion of $3 \mathrm{~mL}$ of $4^{\circ} \mathrm{C}$ Perfadex ${ }^{\circledR}$ solution (Vitrolife Inc., Denver, CO) supplemented with THAM Solution (Vitrolife, Kungsbacka, Sweden), estimating weight-based volume recommendations for pulmonary artery perfusion $(140 \mathrm{~mL} / \mathrm{kg})$ [111]. The chest was then packed with ice and the trachea occluded by silk-suture tie at tidal volume $(7 \mu \mathrm{L} / \mathrm{g}$ body weight) prior to cold static preservation (CSP) for 60 minutes at $4^{\circ} \mathrm{C}$. Mice were then randomized into three experimental groups: 1) CSP alone with no EVLP, 2) EVLP with Steen solution and 3) EVLP with Steen solution supplemented with the highly selective $A_{2 A} R$ 
agonist, ATL1223 (30nM, Lewis and Clark Pharmaceuticals, Charlottesville, VA). ATL1223

was chosen as the $A_{2 A} R$ agonist because we have previously demonstrated that ATL1223 potently attenuates IR injury in mouse models and in a porcine lung transplantation model and because ATL1223 is a more potent and selective $A_{2 A} R$ agonist versus earlier generation agonists (unpublished studies). Mice treated with ATL1223 during EVLP also received ATL1223 treatment (30nM) during the Perfadex flush prior to CSP whereas the EVLP group received vehicle (DMSO) during the flush. CSP lungs, which did not undergo EVLP with Steen solution, underwent immediate functional assessment after re-intubation as described below in order to define the status of the lungs at the initiation of EVLP.

\section{Murine EVLP}

In the two groups that underwent EVLP, mice were re-intubated after CSP to permit mechanical ventilation with room air $(100$ strokes $/$ minute, tidal volume $=7 \mu \mathrm{l} / \mathrm{g}$ body weight, $2 \mathrm{~cm} \mathrm{H}_{2} \mathrm{O}$ PEEP) by anterior tracheostomy. The right ventricle was cannulated for placement onto a murine isolated, lung perfusion apparatus (Hugo Sachs Elektronik, MarchHuggstetten, Germany) as previously described[112]. The inferior vena cava was transected for exsanguination and the left ventricle vented through an apical ventriculotomy. The lungs were perfused with Steen solution at a constant rate of $60 \mu \mathrm{l} / \mathrm{g}$ body weight/minute, an established standard for isolated murine lung perfusion $[4,6]$. Steen solution within the circuit was gradually warmed from $4^{\circ} \mathrm{C}$ to $37^{\circ} \mathrm{C}$ through the circuit water bath, and EVLP continued for 60 minutes. Steen solution perfusate was supplemented with 10,000 IU heparin, 500mg cefazolin and 500mg methylprednisolone per $1500 \mathrm{~mL}$, modeling preclinical and clinical EVLP protocols[103, 105]. Steen solution was also supplemented with vehicle (DMSO) for the EVLP group or with ATL1223 (30nM) for the ATL1223-treated group. 


\section{Lung function}

Pulmonary function and hemodynamic measurements were recorded at the end of 60 minutes of perfusion using the PULMODYN data acquisition system (Hugo Sachs Elektronik) as previously described[4]. To measure lung function of the CSP group, lungs were placed directly on the isolated lung perfusion apparatus and perfused with standard Krebs-Henseleit buffer for a 5-minute equilibration period before data was recorded for an additional 5 minutes. These CSP lungs were utilized to obtain the data shown. A separate group of CSP lungs were perfused with Krebs-Henseleit buffer for one hour and compared to the EVLP and EVLP+ATL1223 groups in order to assess temporal changes in function during perfusion (Figure 13). 


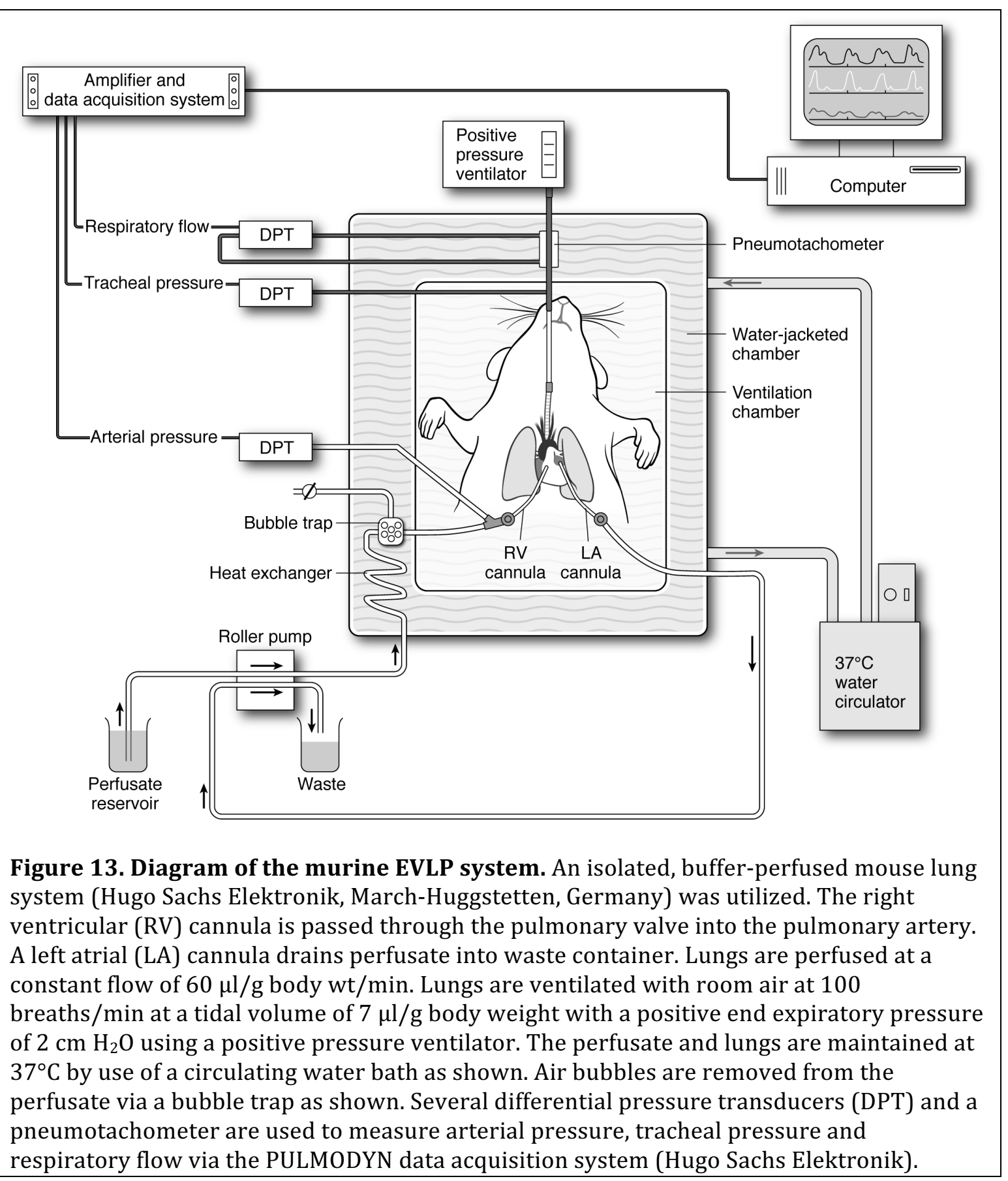




\section{Cytokine measurements}

Using separate groups of animals, proinflammatory cytokines were measured in whole lung lysates using a multiplex cytokine panel assay (Bio-Rad Laboratories, Hercules, CA) as described previously $[4,100]$.

\section{Neutrophil counts}

Using lungs from separate groups of animals, calculation of neutrophil numbers per high-powered field were performed on immunostained lung sections as described previously[4].

\section{Lung wet/dry weight ratio}

Right lungs were weighed and desiccated until a stable dry weight was achieved to calculate the lung wet/dry weight as an indicator of edema.

\section{Statistical Analysis}

Statistical analyses were performed using GraphPad Prism 6.0 software, and data are presented as the mean \pm standard error of the mean. One-way ANOVA with post-hoc Tukey's multiple comparison test was performed to compare experimental groups. Statistical significance was set at $P<0.05$.

\section{RNA isolation and microarray hybridization}

Using separate groups of animals, total RNA was extracted from whole lungs using TRIzol reagent (Life technologies, Carlsbad, CA), following the Affymetrix GeneChip ${ }^{\circledR}$ Expression Analysis Manual (Affymetrix, Santa Clara, CA, USA) guidelines and recommendations. All RNA samples met purity and integrity quality control criteria previously established[113]. Reactions for cDNA synthesis and in vitro transcription for labeled cRNA probe, microarray hybridization, image generation, and probesets reading process were performed as reported previously[113, 114]. In total, twelve Affymetrix GeneChip Mouse Genome 430A 2.0 microarrays were hybridized for three separate groups 
of animals ( $n=4$ /group). After hybridization, each chip was scanned on an Affymetrix GeneChip ${ }^{\circledR}$ Scanner 3000 G7. Raw intensities for every probe were stored in electronic files (.DAT and .CEL formats) by the GeneChip ${ }^{\circledR}$ Operating Software (GCOS).

\section{Microarray Quality Control and Data Analysis}

The hybridized Affymetrix GeneChip Mouse Genome 430A 2.0 microarrays were analyzed using RMAexpress software to normalize probeset data by quantile normalization and summarized with median polish summarization using the Robust Multiarray Average method $[115,116]$. Pairwise comparisons (EVLP vs. CSP and EVLP+ATL1223 vs. CSP) were fit using two-sample $t$-test in the R programming environment[117]. To adjust for the multiple hypothesis tests, the $p$-values were used in estimating the false discovery rate (FDR) using the Benjamini and Hochberg method[118, 119]. $P$-values $\leq 0.001$ under a controlled FDR $<1 \%$ were considered significant. Fold-change values were used for differential expression magnitudes.

Interaction Networks, Functional Analysis, and Upstream regulators.

The Ingenuity Pathway Analysis (IPA, www.ingenuity.com) tool was used to analyze gene ontology and pathways of differential expressed genes. $P$-values $\leq 0.05$ were considered significant. Spreadsheet lists containing probesets ID and fold-changes were generated and uploaded to IPA.

\section{Results}

\section{ATL1223 Improves Lung Function and Reduces Edema During EVLP of DCD Lungs}

DCD lungs undergoing EVLP demonstrated significantly increased pulmonary compliance ( $3.70 \pm 0.13$ vs. $1.88 \pm 0.21 \mu \mathrm{l} / \mathrm{cm} \mathrm{H}_{2} \mathrm{O}$, respectively) and decreased pulmonary artery pressure (9.22 \pm 0.21 vs. $14.14 \pm 0.37 \mathrm{~cm} \mathrm{H}_{2} \mathrm{O}$, respectively) after 1 hour of perfusion compared to CSP lungs (Figure 14). In addition, EVLP significantly reduced pulmonary edema as assessed by wet/dry weight compared to CSP lungs ( $4.58 \pm 0.13$ vs. $5.15 \pm 0.18$, 
respectively) (Figure 14). Furthermore, EVLP with Steen solution supplemented with ATL1223 significantly increased pulmonary compliance $(5.17 \pm 0.15$ vs. $3.70 \pm 0.13 \mu \mathrm{l} / \mathrm{cm}$ $\mathrm{H}_{2} \mathrm{O}$, respectively) and reduced pulmonary artery pressure $(7.79 \pm 0.22$ vs. $9.22 \pm 0.21 \mathrm{~cm}$ $\mathrm{H}_{2} \mathrm{O}$, respectively) when compared to EVLP alone after 1 hour of perfusion (Figure 14). Temporal measurements of lung function during the 1 hour perfusion period demonstrate that function in the EVLP + ATL1223 group began to significantly diverge from the EVLP group by 30 minutes of perfusion and that lung function in the CSP group did not significantly change when perfusion was extended to one hour. In accordance with functional improvement, EVLP with ATL1223 also significantly reduced pulmonary edema compared to EVLP alone (4.08 \pm 0.10 vs. $4.58 \pm 0.13$, respectively). 

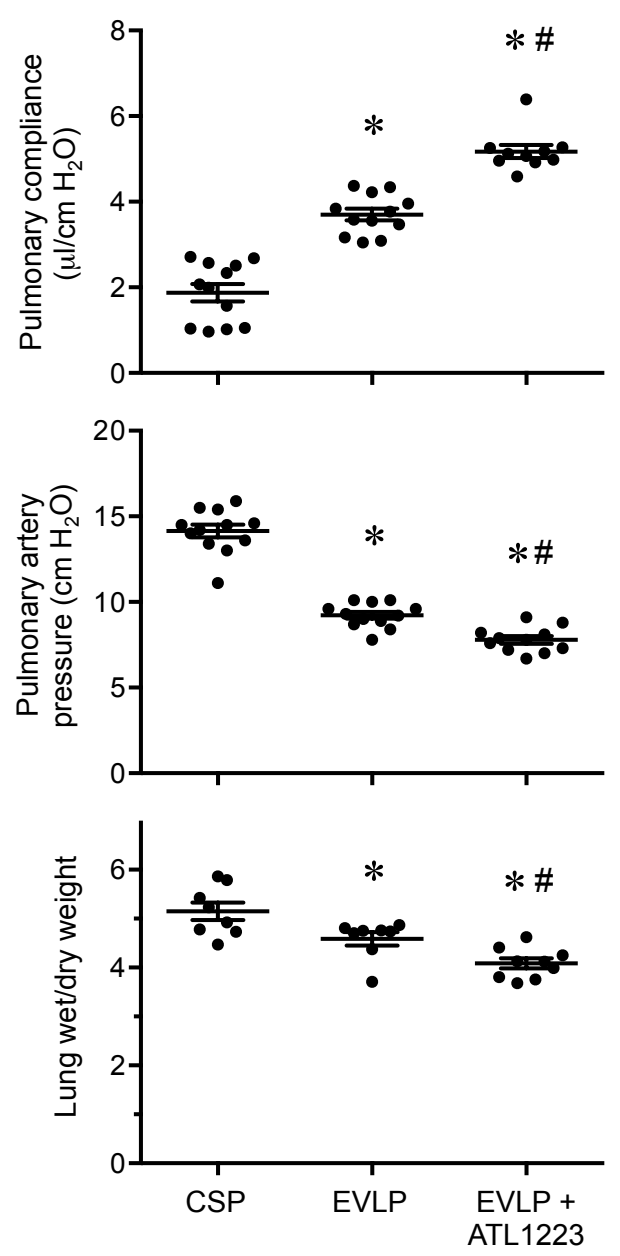

Figure 14. EVLP-directed delivery of ATL1223 improves function and reduces edema in DCD lungs. Compared to lungs after cold static preservation (CSP), EVLP significantly increased pulmonary compliance and reduced pulmonary artery pressure and wet/dry weight (edema). ATL1223 treatment during EVLP provided further, significantly improved lung function and reduced edema. One-way ANOVA with post-hoc Tukey's multiple comparison test was performed to compare groups. Results are presented as mean \pm SEM. ${ }^{*} p<0.05$ versus CSP; ${ }^{\#}<0.05$ versus EVLP; $n=10-12$ /group (pulmonary compliance and pulmonary artery pressure); n=8-9/group (wet/dry weight).

\section{EVLP with ATL1223 Reduces Pro-Inflammatory Cytokine Expression}

Expression of CXCL1, CCL2 and TNF- $\alpha$ were reduced in lungs following EVLP, although this did not reach significance (Figure 15). Treatment with ATL1223 during EVLP significantly reduced the levels of CXCL1, CCL2 and TNF- $\alpha$ versus EVLP alone. CXCL1 results for CSP, EVLP and EVLP+ATL1223 groups were: 78.9 $\pm 37.6,19.6 \pm 4.4$ and $4.8 \pm 0.4 \mathrm{pg} / \mathrm{ml}$, 
respectively. CCL2 results for CSP, EVLP and EVLP+ATL1223 groups were: 319.7ะ78.7, 208.4 \pm 46.2 and $89.8 \pm 20.2 \mathrm{pg} / \mathrm{ml}$, respectively. TNF- $\alpha$ results for CSP, EVLP and EVLP+ATL1223 groups were: $268.4 \pm 24.6,210.0 \pm 39.5$ and $150.4 \pm 19.0 \mathrm{pg} / \mathrm{ml}$, respectively.
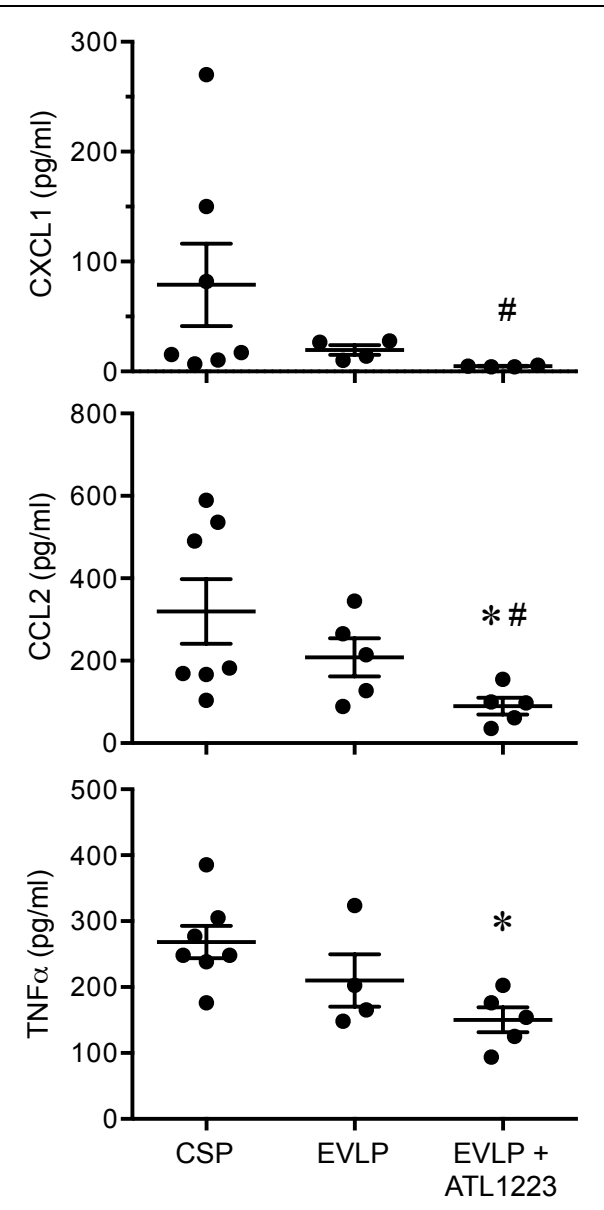

Figure 15. EVLP-directed delivery of ATL1223 reduces proinflammatory cytokine production in DCD lungs. Compared to lungs after cold static preservation (CSP), expression of CXCL1, CCL2 and TNF- $\alpha$ were slightly, but not significantly, reduced by EVLP. ATL1223 treatment during EVLP resulted in significant reductions in expression of CXCL1, CCL2 and TNF- $\alpha$. One-way ANOVA with post-hoc Tukey's multiple comparison test was performed to compare groups. Results are presented as mean \pm SEM. ${ }^{*} p<0.05$ versus CSP; ${ }^{\#} p<0.05$ versus EVLP; $\mathrm{n}=4-7$ /group. 


\section{EVLP with ATL1223 Reduces Lung Neutrophil Numbers}

Immunostaining of lung sections revealed a small decrease in neutrophil numbers after EVLP versus CSP, although this was not significant (Figure 16). However, EVLP with ATL1223 significantly reduced neutrophil numbers versus both CSP and EVLP groups (2.06 \pm 0.34 vs. $5.42 \pm 1.13$ and $4.06 \pm 0.77$, respectively).

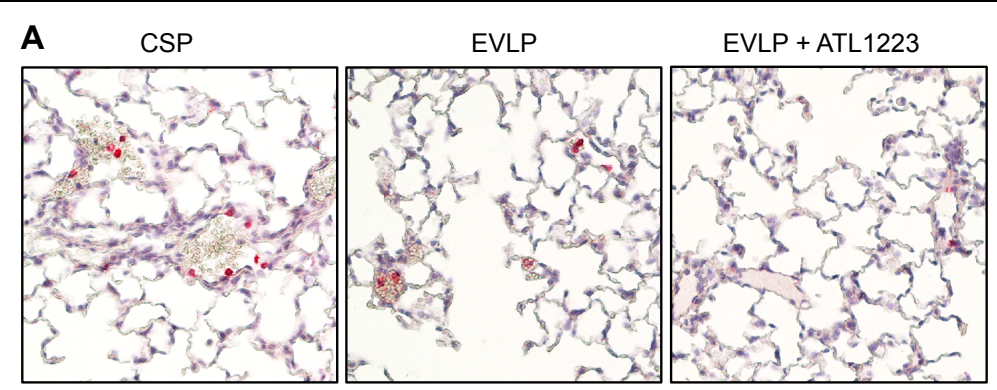

B

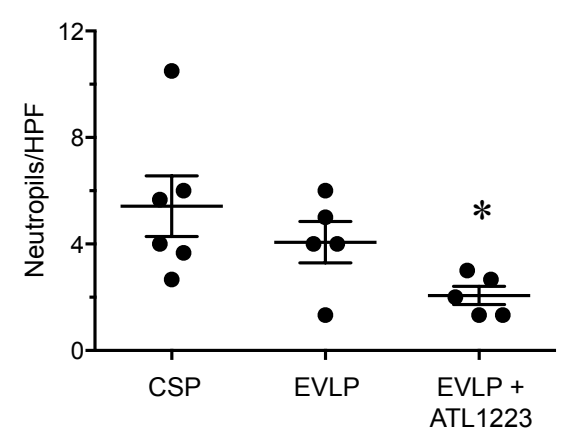

Figure 16. EVLP-directed delivery of ATL1223 depletes pulmonary neutrophils. (A) Representative lung sections (20x magnification) showing immunostaining for neutrophils (red staining). (B) Quantitation of the number of neutrophils per high-powered field (HPF) in immunostained lung sections. EVLP with ATL1223 resulted in significantly fewer neutrophils/HPF versus cold static preservation (CSP) and EVLP alone. One-way ANOVA with post-hoc Tukey's multiple comparison test was performed to compare groups. Results are presented as mean \pm SEM. ${ }^{*} p<0.05$ versus all; $n=5-6 /$ group.

\section{Gene Expression Profiles in DCD Lungs After EVLP}

EVLP of DCD lungs resulted in the differential expression of 1,594 genes $(1,762$

probesets) compared to CSP (Figure 17A). Core analysis was performed to interpret the data set in the context of biological processes, canonical pathways and molecular networks. The top two networks identified from the dataset included 1) connective tissue development and function, tissue morphology, cell cycle (score 36 , score $\geq 5$ was considered 
significant) and 2) molecular transport, skeletal and muscular system development and function, free radical scavenging (score 31).

A

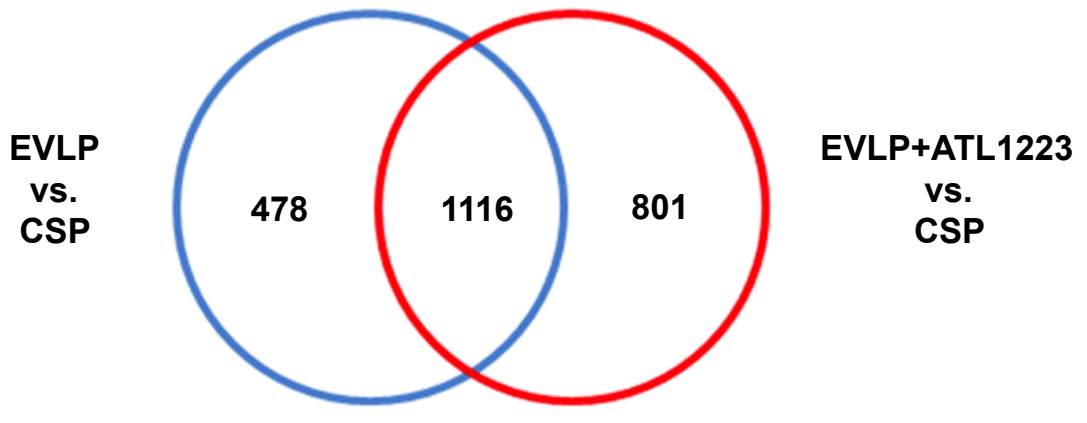

B
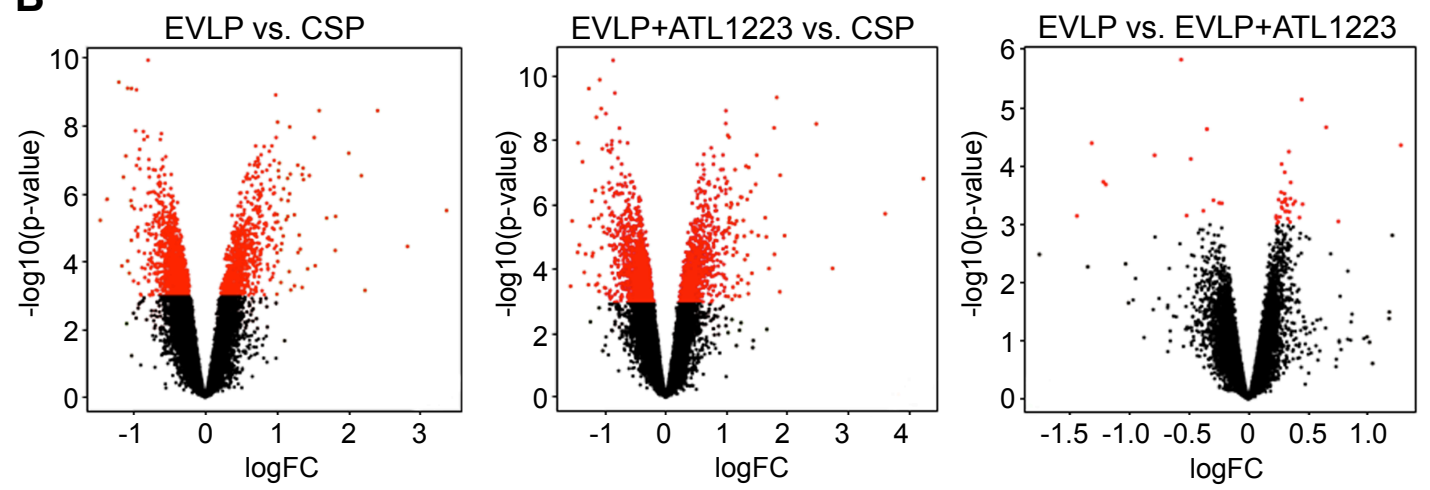

Figure 17. Differentially expressed genes among study groups as a function of EVLPmediated rehabilitation strategy for DCD lungs. (A) Venn diagram showing numbers of differentially expressed genes in lungs from EVLP and EVLP+ATL1223 groups when compared to the cold static preservation (CSP) control group (FDR 1\%). (B) Volcano plots depicting the gene expression profile of pairwise comparisons of indicated groups. Each dot represents a unique, differentially expressed probeset. Significant probesets [p-value $\leq$ 0.001 or $-\log 10$ (p-value) $\geq 3$ ] are illustrated by red dots.

From the analysis of molecular and cellular functions using the differentially expressed genes between the EVLP vs. CSP groups, cell death and survival ( $p$-value range=1.15E-09-1.06E-02, 299 genes), gene expression ( $p$-value range=1.39E-08-8.38E-04, 179 genes) and lipid metabolism ( $p$-value range=4.59E-08-1.07E-02, 153 genes) were identified as the more relevant between the groups. A total of 123 canonical pathways were identified as significant and associated with the EVLP gene expression profile when 
compared to CSP $(p<0.05)$. From these canonical pathways, ten selected pathways focusing on inflammation and innate immune responses are highlighted in Figure 18A including IL1 signaling ( $p$-value=3.5E-04), IL-8 signaling ( $p$-value=5.0E-04) and IL-17 signaling $(p$ value=1.7E-02). Further analysis of the IL-1 and IL-8 signaling pathways showed an important number of genes down-regulated by EVLP including IL6ST, SOCS3, PIK3CA, PIK3C2A, PIK3R1, MAPK13, TRAF6, FOS, JUN, CRP, PIK3CD, TNFRSF1B, IL1RAP, TNF and ATM, among others. Overall, as shown in Figure 18A, these pathways showed a higher percentage of genes down-regulated in the EVLP group. 


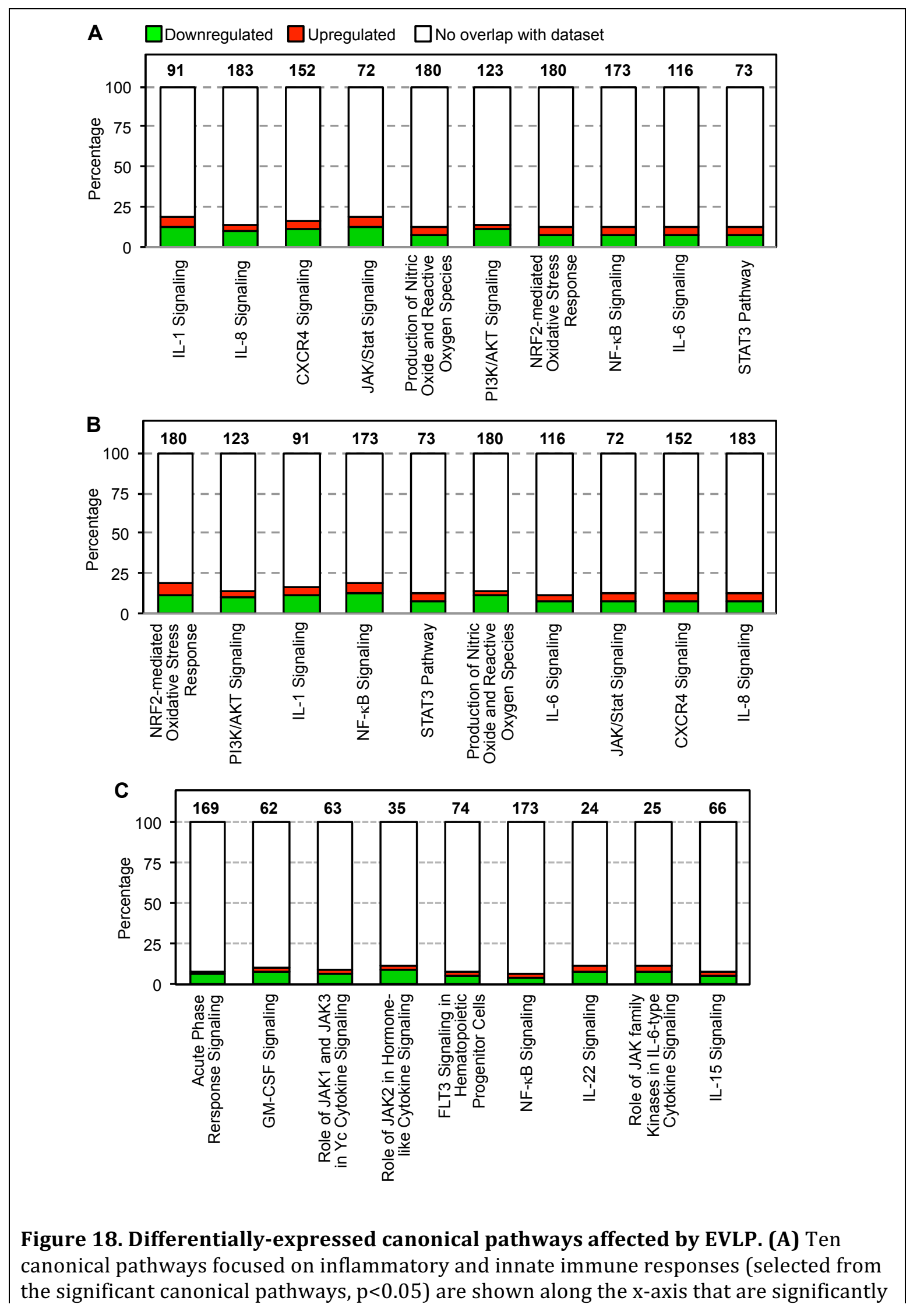


different between EVLP versus CSP. (B) Ten canonical pathways focused on inflammatory and innate immune responses (selected from the significant canonical pathways, $p<0.05$ ) are shown along the $x$-axis that are significantly different between EVLP+ATL223 versus CSP. (C) The canonical pathways resulting from the evaluation of genes uniquely differentially expressed in EVLP+ATL1223 vs. CSP (801 genes, FDR 1\%). The total number of genes associated with each pathway is listed above each bar. Green and red bars indicate the percentage (y axis) of those genes down- or up-regulated, respectively, as calculated by (\# genes in a given pathway that meet cutoff criteria) / (total \# genes that comprise that pathway). Pathways are presented left-to-right from highest to lowest significance.

\section{Differential Gene Expression Analysis in DCD Lungs After EVLP+ATL1223}

A comparison between the EVLP+ATL1223 and CSP groups resulted in a total of 1,917 differentially expressed genes, with 1,116 genes overlapping with the EVLP vs. CSP comparison analysis (Figure 17A). This indicates an important overlap between affected biological pathways in both EVLP conditions. However, the number of unique genes affected in the EVLP+ATL1223 vs. CSP analysis was higher than in the EVLP vs. CSP analysis. A direct comparison analysis of EVLP vs. EVLP+ATL1223 revealed a limited number of genes differentially expressed between these groups even when a flexible cutoff for significance ( $p$-value<0.001) was used (Figure 17B).

When comparing EVLP+ATL1223 to CSP, the top molecular and cellular functions associated with the statistical differentially expressed genes included 1) gene expression ( $p$ value range=3.7E-22-3.2E-04, 427 genes), 2 ) cellular growth and proliferation ( $p$-value range $=3.3 \mathrm{E}-20-3.1 \mathrm{E}-04,627$ genes) and 3$)$ cell death and survival ( $p$-value range $=1.1 \mathrm{E}-16-$ 3.7E-04, 596 genes). A total of 166 canonical pathways were identified as significant and associated with the EVLP+ATL1223 gene expression profile when compared to CSP $(p<0.05)$. From these canonical pathways, ten selected pathways focusing on inflammation and innate immune responses are highlighted in Figure 18B including IL-1 signaling ( $p$ value=3.0E-03), IL-8 signaling ( $p$-value=2.7E-02) and IL-17 signaling ( $p$-value=3.6E-02). Interestingly, the evaluation of unique genes differentially expressed in EVLP+ATL1223 vs.

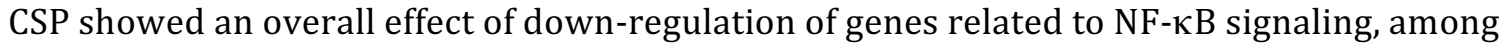


other pathways (9 in total), with most of these genes down-regulated in EVLP+ATL1223 vs. CSP (Figure 18C).

\section{Identification of Differential Gene Expression Profiles Associated with EVLP+ATL1223 versus EVLP Alone}

To evaluate differences strictly associated with ATL1223 treatment during EVLP, we compared EVLP and EVLP+ATL1223 to the same CSP group and performed comparison analysis using IPA. From the analysis, a noticeably greater number of IL-1 signaling genes were down-regulated in the EVLP+ATL1223 group including down-regulation of ADCY9, ECSIT, IRAK1, MAPK12 and TOLLIP; indicating a greater effect in the number of inflammatory genes decreased with the use of ATL1223. Also, a similar effect was observed for acute phase response signaling with an additional number of genes down-regulated in the EVLP+ATL1223 group including PTPN11, MAP3K5, CP, PDPK1, SOCS5, MAPK12, TRADD, MAP3K7 and IRAK1 (data not shown).

\section{Discussion}

Our group and others have demonstrated in preclinical models that DCD lungs with extended warm ischemic times (up to 60 minutes) may be rehabilitated by EVLP to an acceptable state for transplantation $[106,120]$. These results have been furthered by clinical studies in which initially rejected human donor lungs have been rehabilitated to acceptable standards for transplantation $[121,122]$. While these results support a promising future for use of DCD lungs in successful transplantation, not all lung grafts are recoverable and many experience a decrease in oxygenation capacity with an increase in interstitial edema during EVLP [123]. The future application of EVLP is, therefore, dependent on early, targeted therapy and the establishment of predictive standards for post-transplantation lung function. For example, experimental studies have identified nebulized arginase inhibitor and adenoviral vector delivery of IL-10 as potential 
rehabilitative strategies during EVLP [124, 125]. Furthermore, an understanding of the mechanisms that underlie EVLP-mediated rehabilitation would lead to the advancement of this technique for lung transplantation.

The present study introduces a novel murine model of EVLP of DCD lungs, which provides a fundamental framework for future studies aimed toward identifying potential predictive markers of PGD after EVLP and transplantation as well as the assessment of novel targeted therapies for EVLP-mediated donor lung rehabilitation. Importantly, our study demonstrates that EVLP of DCD lungs leads to significantly improved function and reduced edema.

$A_{2 A} R$ agonism has been established as an effective strategy for the prevention of IR injury after experimental lung transplantation $[99,108-110,120]$. Recently, using a porcine model, our group has demonstrated that $\mathrm{A}_{2 \mathrm{~A}} \mathrm{R}$ agonist therapy provides effective EVLPmediated rehabilitation of heart-beating donor lungs after extended cold ischemic preservation [126]. While this study demonstrated the potential for translation of this therapy to clinical transplantation, no comparative analysis of EVLP-mediated $A_{2 A} R$ agonist treatment has been previously performed in DCD lungs. Thus, the present study utilizes the murine EVLP model to demonstrate enhanced rehabilitation of DCD lungs via $A_{2 A} R$ agonist therapy, which results in significantly improved lung function as well as reduced edema and inflammation.

Although EVLP improved function of murine DCD lungs, EVLP resulted in small reductions of pro-inflammatory cytokine levels and neutrophil counts that were not statistically significant. It is possible that EVLP with Steen solution largely preserves endothelial function to attenuate edema and dysfunction, resulting in more indirect effects on cytokine expression during reperfusion. Importantly, EVLP with $A_{2 A} R$ agonist treatment 
significantly decreased pro-inflammatory cytokine levels and neutrophil numbers, two established hallmarks of lung IR injury and PGD [127].

Because lungs are perfused with acellular Steen solution, neutrophil numbers in lungs were overall lower than what is typically observed after IR in vivo [4]. As shown, it is likely that most observed neutrophils are marginated (i.e. temporarily adhered to endothelium). We have previously shown that buffer perfusion of isolated murine lungs does not readily wash away significant numbers of marginated neutrophils [6]. Although EVLP may have slightly decreased the number of marginated neutrophils, the inclusion of ATL1223 with EVLP resulted in significantly fewer neutrophils. This suggests that $A_{2 A} R$ agonism reduces the adhesion of marginated neutrophils, which is supported by studies showing that $A_{2 A} R$ agonists modulate adhesion molecule expression [128] and inhibit neutrophil transepithelial migration [129]. Because neutrophil activation and infiltration is a key component of IR injury, the flushing away of greater numbers of marginated neutrophils in DCD lungs by EVLP-mediated $\mathrm{A}_{2 \mathrm{~A}} \mathrm{R}$ agonist delivery would be beneficial in attenuating IR injury after transplantation.

A traditional measure of lung function during EVLP, often used to predict successful transplantation, has been the partial pressure of oxygen $\left(\mathrm{PO}_{2}\right)$ in the EVLP perfusate. However, this may be misleading since Yeung et al. used a preclinical study of high-risk donor lungs to demonstrate a higher predictive value for decreased compliance and increased airway pressure than $\mathrm{PO}_{2}$ during EVLP [130]. Thus Yeung et al. demonstrated that one cannot place the full emphasis of lung assessment on $\mathrm{PO}_{2}$ during EVLP and that ex vivo $\mathrm{PO}_{2}$ may not be the first indication of lung injury [130]. Although $\mathrm{PO}_{2}$ levels during EVLP were not measured in the present study, our results support the concept that pulmonary compliance and pulmonary artery pressure may be useful and important physiologic predictors of graft function. 
Gene expression analysis revealed a large number of canonical pathways, including many associated with inflammation and innate immune responses, that are significantly affected by EVLP, with the overall affect being downregulation of most genes in these pathways. The induction of inflammatory responses after reperfusion is believed to be the major contributor to IR injury and PGD after transplantation, and the present study suggests that proinflammatory responses in DCD lungs occur quickly after perfusion and that EVLP with Steen solution effectively attenuates these responses via modulation of the expression of many proinflammatory genes. Although treatment with ATL1223 during EVLP resulted in a limited number of differentially expressed genes compared to EVLP without ATL1223, a larger number of canonical pathways overall were significantly affected after EVLP with ATL1223. Gene analysis demonstrated the modulation of multiple and overlapping inflammatory pathways after EVLP with or without ATL1223. It is possible that some of these may be useful biomarkers to assess inflammation in lungs during EVLP as a potential means to gauge the acceptability of DCD lungs for successful transplantation.

There are several limitations to the current study. First, the circuit design is 'open' rather than 'closed' as typically utilized in clinical EVLP protocols. This may actually introduce the potential for improved efficacy, as the murine model drains perfusate through the left atrium and may more effectively clear both pro-inflammatory cytokines and neutrophils that would persist in a closed circuit. While recognizing this limitation, many advocate a standardized approach of Steen solution replacement during EVLP [103], and the present study supports the potential benefit of this approach. Second, murine lungs were assessed ex vivo but were not transplanted. While murine models of transplantation exist, the focus of this initial study was to evaluate lung function and injury during EVLP, and thus these results could not be translated to transplanted lungs. Third, EVLP was performed for 1 hour whereas clinical EVLP is typically performed for 4 hours (or even 
longer). Although 1 hour of EVLP was sufficient for significant improvements in lung function and edema, it is possible that a longer period of EVLP would lead to more significant effects on inflammation markers such as cytokines or gene expression. Preliminary studies indicated that perfused lungs typically stabilize within 30 minutes and remain stable throughout 1 hour of perfusion and beyond, thus we chose to assess lungs after 1 hour. Fourth, it is possible that anti-inflammatory effects of methylprednisolone could have an influence on lung inflammation (e.g. cytokine levels) during EVLP. Steen solution was supplemented with methylprednisolone (as well as heparin and cefazolin) in order to model what is used in many preclinical and clinical EVLP protocols. However, both the EVLP and EVLP+ATL1223 groups of lungs were perfused with identical perfusate (+/ATL1223) and thus were directly comparable. Finally, it is possible that the beneficial effects of ATL1223 during EVLP could be due to the vasodilatory actions of $A_{2 A} R$ agonism as shown by many studies. However, this is unlikely since the dose of $A_{2 A} R$ agonist required for cardiovascular effects are much higher than the dose required for inhibition of inflammation, and the dose of ATL1223 utilized in the present study was based on wellestablished doses used in prior studies that prevent IR injury but do not have significant cardiovascular effects $[108,109,131]$.

In conclusion, the present study demonstrates that EVLP provides an effective platform for the rehabilitation of DCD lungs in a novel murine model. EVLP-mediated therapy with $A_{2 A} R$ agonist results in significantly enhanced protection as demonstrated by reductions in pulmonary dysfunction, edema, pro-inflammatory cytokines and neutrophil numbers. Additionally, a decrease in the expression of genes associated with inflammation was observed after EVLP. The murine EVLP model provides a reproducible and effective means for experimental, mechanistic studies of novel EVLP-directed therapies that may help in the identification of predictive biomarkers of lung function after EVLP and 
transplantation. Future translation of EVLP and $\mathrm{A}_{2 \mathrm{~A}} \mathrm{R}$ agonist therapy to DCD lungs could greatly impact all lung transplant recipients by not only increasing the donor pool size to reduce the wait-list mortality but may also attenuate PGD leading to improved short- and long-term outcomes for lung transplant patients. 


\section{Ex Vivo Rehabilitation of Non-Heart-Beating Donor Lungs in a Preclinical Porcine Model: Delayed Perfusion Results in Superior Lung Function}

Matthew L. Stone MD*a, Daniel P. Mulloy MD*a, Ivan K. Crosby MBBSa, Damien J. LaPar MDa, Ashish K. Sharma MBBS a, David V. Webb MDb, Christine L. Lau MDa, Victor E. Laubach PhDa, and Irving L. Kron MDa

*Equal contributors

Departments of aSurgery and ${ }^{\mathrm{b}}$ Pathology University of Virginia, Charlottesville, Virginia

Stone ML, Mulloy DP et al. J Thorac Cardiovasc Surg 2012;144(5):1208-15. 


\begin{abstract}
Objectives: Ex vivo lung perfusion (EVLP) is a promising modality for the evaluation and treatment of marginal donor lungs. The optimal timing of EVLP initiation and potential for rehabilitation of donor lungs with extended warm-ischemic times is unknown. This study compares the efficacy of different treatment strategies for uncontrolled non-heart-beating donor lungs.
\end{abstract}

Methods: Mature swine underwent hypoxic arrest followed by 60 minutes of no-touch warm-ischemia. Lungs were harvested and flushed with $4^{\circ} \mathrm{C}$ Perfadex ${ }^{\circ}$. Three groups ( $\mathrm{n}=5$ /group) were stratified according to preservation method: cold-static preservation (CSP: $4 \mathrm{hrs} 4^{\circ} \mathrm{C}$ storage), immediate EVLP (I-EVLP: $4 \mathrm{hrs}$ EVLP at $37^{\circ} \mathrm{C}$ ), and delayed EVLP (D-EVLP: $4 \mathrm{hrs}$ cold storage followed by $4 \mathrm{hrs} \mathrm{EVLP).} \mathrm{EVLP} \mathrm{groups} \mathrm{were} \mathrm{perfused} \mathrm{with}$ Steen solution ${ }^{\mathrm{TM}}$ supplemented with heparin, methylprednisolone, cefazolin, and an adenosine 2A receptor agonist. Lungs then underwent allotransplantation and four hours of recipient reperfusion prior to allograft assessment for resultant ischemia-reperfusion injury.

Results: Donor blood oxygenation $\left(\mathrm{PO}_{2}: \mathrm{FiO}_{2}\right)$ prior to euthanasia was not different between groups. Oxygenation after transplantation was significantly higher in the D-EVLP group compared to the I-EVLP or CSP groups. Mean airway pressure, pulmonary artery pressure, and expression of IL-8, IL-1 $\beta$, and TNF- $\alpha$ were all significantly reduced in the DEVLP group. Importantly, post-transplant oxygenation exceeded acceptable clinical levels only in D-EVLP lungs.

Conclusions: Uncontrolled non-heart-beating donor lungs with extended warm-ischemia can be reconditioned for successful transplantation. The combination of CSP and EVLP present in the D-EVLP group was necessary to obtain optimal post-transplant function. This finding, if confirmed clinically, will allow expanded use of non-heart-beating donor lungs. 


\section{Introduction}

Lung transplantation is a lifesaving treatment for patients with end-stage pulmonary disease; however, its success is limited by significant donor organ shortages. To address this growing problem, many centers now utilize a limited number of marginal or extended criteria heart-beating (HB) donor lungs. Recently, transplantation of lungs from non-heart-beating (NHB) donors has gained renewed interest as a potential mechanism to alleviate donor organ shortages. NHB donors are classified by Maastricht category according to the circumstances of expiration (I - dead on arrival to the hospital, II - failed resuscitation, III - withdrawal of life support, awaiting cardiac arrest, IV - cardiac arrest in brain-dead donor), and further described as uncontrolled (categories I and II) and controlled (categories III and IV) donors[132]. Unfortunately, several case series using NHB donor lungs for transplantation have shown higher rates of primary graft dysfunction, bronchiolitis obliterans, and mortality in comparison to HB donor lungs, and accurately predicting post-transplantation function of NHB donors lungs has proven difficult in part due to variation in warm ischemic times[101,133, 134].

Ex vivo lung perfusion (EVLP) is a technique of normothermic acellular lung perfusion for both donor lung assessment and rehabilitation ex vivo[103, 135]. The promise of this technique has been demonstrated in recent human clinical trials with marginal donor lungs (Maastricht III and IV), yet questions remain regarding the optimal timing of EVLP, potential application as a platform for therapeutic delivery, and rehabilitation potential for Maastricht category I and II donor lungs[21, 97].

The purpose of this study was to determine, using a preclinical porcine transplant model, if lungs from uncontrolled NHB donors (Maastricht category I) with extended warm ischemic times could be rehabilitated to an acceptable functional status for subsequent successful transplantation. We hypothesized that initiation of EVLP immediately after NHB 
donor lung explantation would minimize cold ischemic time, allow for rapid initiation of directed donor lung treatment, and result in superior outcomes after lung transplantation when compared to either cold static preservation alone or delayed initiation of EVLP after a period of cold static preservation.

\section{Materials and Methods}

\section{Animals}

The University of Virginia's Institutional Animal Care and Use Committee reviewed and approved all aspects of this study. Humane animal care was observed in accordance with the "Guide for Care and Use of Laboratory Animals" (National Institutes of Health publication no. 85-23, revised 1985).

\section{Study Groups}

Mature domestic swine of both sexes $(20-38 \mathrm{~kg})$ were randomized throughout the study among 3 different study groups ( $n=5$ /group) stratified according to donor lung preservation method. Donor swine from all groups underwent hypoxic arrest followed by 60 minutes of no-touch warm ischemia. The cold-static preservation (CSP) group underwent lung procurement and 4 hours of storage in $4^{\circ} \mathrm{C}$ Perfadex ${ }^{\circledR}$ (Vitrolife Inc., Denver, CO), a commercially available preservative solution widely used for human lung transplantation. Left donor lungs were subsequently transplanted into size-matched recipients. The CSP group served as the ischemia-reperfusion injury control. The immediate EVLP (I-EVLP) group underwent lung procurement and 4 hours of immediate normothermic EVLP, followed by transplantation. The delayed EVLP (D-EVLP) group received a combination of both strategies and underwent lung procurement, 4 hours of storage in $4^{\circ} \mathrm{C}$ Perfadex, followed by 4 hours of normothermic EVLP prior to transplantation. After left lung transplantation, lungs of all animals were perfused in vivo for 
a continuous 4-hour period, after which parameters of lung function and injury were assessed as described below.

\section{Porcine Arrest and Donor Lung Procedure}

Donor animals were anesthetized with ketamine $(50 \mathrm{mg} / \mathrm{kg})$ and xylazine (5 $\mathrm{mg} / \mathrm{kg}$ ), ventilated with room air, and intubated. After intubation, anesthesia was maintained for 10 minutes with 3\% isoflurane and the lungs were ventilated with $100 \%$ fraction of inspired oxygen $\left(\mathrm{FiO}_{2}\right)$ using a volume control ventilator (Harvard Apparatus, Boston MA) at a tidal volume of $8 \mathrm{~mL} / \mathrm{kg}$, respiratory rate of $14-18$ breaths/minute, and a positive end-expiratory pressure (PEEP) of $5.0 \mathrm{~cm} \mathrm{H}_{2} \mathrm{O}$. Each swine was placed in the supine position and continuous electrocardiographic monitoring was initiated. After 10 minutes, a baseline arterial blood gas sample was obtained via percutaneous withdrawal from the carotid artery. Following arterial blood gas measurement, the ventilator was disconnected, the endotracheal tube was occluded, and the animal was euthanized via hypoxic arrest. Electrocardiographic activity was monitored until full cessation of electric activity occurred at which point the animal was declared dead. Following expiration, the animal was kept at room temperature for a 60-minute no-touch period. Ventilation was then resumed per pre-euthanasia settings, and lung harvest was performed using a standard operative technique as previously described[109].

Briefly, donor animals underwent a median sternotomy and pericardiotomy to expose the heart, great vessels, and both lungs. The main pulmonary artery (PA) was cannulated with a cardioplegia cannula (Sarns, Ann Arbor, MI), the PA was cross-clamped proximal to this point, and prostaglandin E1 $(10 \mathrm{mg} / \mathrm{kg})$ was injected directly into the main PA. The left atrial appendage was incised, the superior and inferior vena cavae were ligated, and antegrade flushing of both lungs was performed with $1.5 \mathrm{~L}$ of $4^{\circ} \mathrm{C}$ Perfadex. Due to the prolonged warm ischemic time, clot formation was commonly encountered in the left 
atrium and pulmonary veins. Heparin $(10,000 \mathrm{IU})$ was added to the Perfadex flush for all animals. During the flush, the left atrium was incised and efforts were made to manually remove as much clot as possible from the atrium and pulmonary veins (Figure 19A). Upon completion of the antegrade flush, both lungs were inflated with $100 \% \mathrm{FiO}_{2}$ to tidal volume and the heart and both lungs were explanted en bloc. The heart was then removed with care to preserve a generous atrial cuff and the lungs were retrograde flushed with an additional $500 \mathrm{~mL}$ of $4{ }^{\circ} \mathrm{C}$ Perfadex to remove remaining clot from the pulmonary vasculature. For the CSP and D-EVLP groups, the lungs were placed in a standard preservation bag and stored in $4^{\circ} \mathrm{C}$ Perfadex. For the I-EVLP group, lungs were placed directly on ex vivo perfusion. 

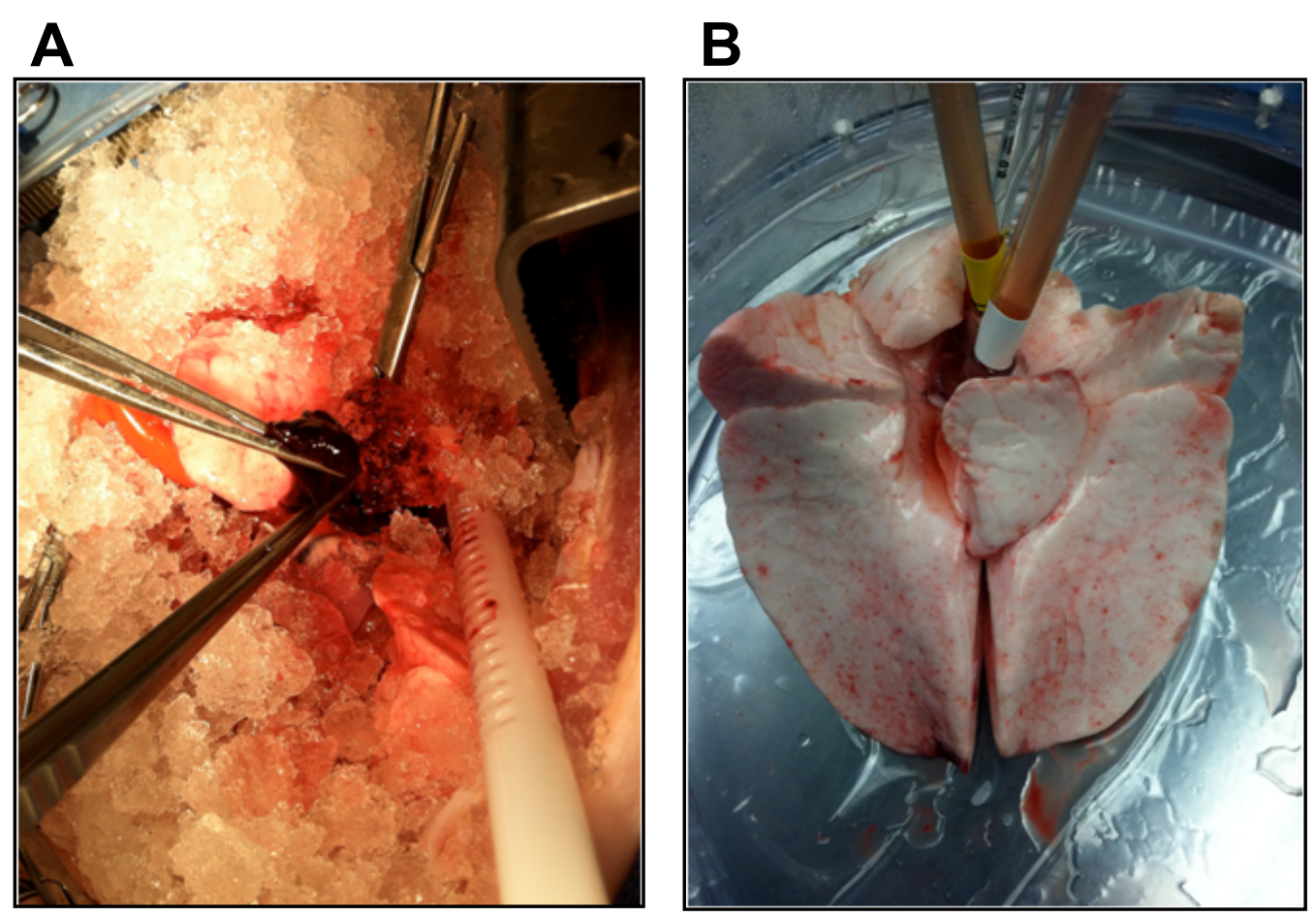

Figure 19. Non-heart-beating donor lung harvest and ex vivo lung perfusion (EVLP) procedures. A. Fresh clot being removed from the donor left atrium and pulmonary veins during the antegrade lung flush. B. Donor lungs pictured during EVLP with the pulmonary artery cannula, left atrial cannula, and endotracheal tube secured in place.

\section{Porcine Ex Vivo Lung Perfusion}

EVLP was performed as described previously [126] and based upon earlier studies by Cypel et al.[103]. Briefly, a funnel-shaped plastic cannula (Vitrolife) was sewn to the left atrial cuff, a plastic cannula (Vitrolife) was secured into the main pulmonary artery (PA), and an 8-0 endotracheal tube with the balloon removed was secured into the trachea (Figure 19B). The EVLP circuit consisted of a bypass centrifugal pump (Medicus, Minneapolis, MN), membrane oxygenator, heat exchanger, venous reservoir (Sorin Group, Arvada, CO), and polyethylene tubing. Lungs were transferred to an XVIVO chamber (Vitrolife), and retrograde flow was initiated through the left atrium to de-air the pulmonary vasculature and flush any remaining clot. The PA cannula was then connected and antegrade flow was commenced at $0.1 \mathrm{~L} / \mathrm{min}$. EVLP was performed using acellular 
Steen solution ${ }^{\mathrm{TM}}$ (Vitrolife Inc., Denver, CO), a commercially available preservative solution designed for ex vivo lung assessment, supplemented with 10,000 IU heparin (APP Pharmaceuticals, Schaumburg, IL), 500 mg cefazolin (Apotex Corp., Weston, FL), 500 mg methylprednisolone (Pfizer, New York, NY), and $3.0 \mathrm{ng} / \mathrm{kg} / \mathrm{min}$. continuous infusion of ATL1223 (Dogwood Pharmaceuticals, Charlottesville, VA), a selective adenosine 2A receptor (A2AR) agonist. ATL-1223 was included to optimize the rehabilitative potential of EVLP because we have previously established the potent anti-inflammatory effects of A2AR agonism in lung ischemia-reperfusion injury and the potential protective advantages afforded with EVLP-directed A2AR agonism treatment $[99,100,108,126,136]$. The perfusate was slowly warmed to $37^{\circ} \mathrm{C}$ over a 30 -minute period as the flow was titrated up to the target of $40 \%$ of the estimated cardiac output (estimated cardiac output $=100$ $\mathrm{ml} / \mathrm{kg}$ ). When the perfusate reached $32^{\circ} \mathrm{C}$, ventilation was initiated with room air at a tidal volume of $8 \mathrm{~mL} / \mathrm{kg}$, respiratory rate of 8 breaths per minute, and a positive end-expiratory pressure (PEEP) of $5.0 \mathrm{~cm} \mathrm{H} \mathrm{H}_{2} \mathrm{O}$. After initiation of ventilation, a mixture of $6 \% \mathrm{O}_{2}, 8 \% \mathrm{CO}_{2}$, and $86 \% \mathrm{~N}_{2}$ was infused into the membrane oxygenator to de-oxygenate the pulmonary artery perfusate and allow for accurate measurement of lung oxygenation capability. At 1 and 4 hours after initiation of EVLP, the lungs were ventilated with $100 \% \mathrm{FiO}_{2}$ for 10 minutes and a sample of the perfusate was taken from the left atrial return for arterial blood gas analysis. At the conclusion of the 4-hour EVLP period, the lungs were removed from the EVLP circuit and an antegrade flush was performed with $500 \mathrm{~mL}$ of $4{ }^{\circ} \mathrm{C}$ Perfadex. The lungs were separated and the right lung was discarded, while the left lung was stored in $4^{\circ} \mathrm{C}$ Perfadex prior to transplantation.

\section{Porcine Left Lung Recipient Transplantation}

Transplantation of the left lung was performed as described previously[109]. Briefly, a left thoracotomy and left pneumonectomy were performed in a size-matched 
recipient swine after heparin administration (5,000 IU). The donor lung was then brought into the field and the donor-to-recipient left bronchus anastomosis was completed in continuous fashion followed by the donor-to-recipient pulmonary artery anastomosis. A portion of the left atrial appendage was then isolated with a side-biting vascular clamp, the atrium was incised, and the donor atrial cuff was then anastomosed to the recipient atrial appendage in continuous fashion. The vascular and airway clamps were then removed to establish reperfusion and ventilation of the transplanted lung.

\section{Lung Physiology}

All transplanted lungs underwent 4 hours of in vivo reperfusion. During reperfusion, hourly arterial blood gas measurements were obtained in addition to mean arterial pressure, heart rate, and mean pulmonary artery pressure via a Swan-Ganz catheter. Pulmonary function post-transplantation was additionally evaluated with mean airway pressure measurements obtained utilizing a pressure monitoring line attached to the endotracheal tube. After 3.5 hours, the endotracheal tube was advanced into the left main-stem bronchus and the right main pulmonary artery was occluded by a preplaced vessel loop, thereby establishing isolated perfusion and ventilation of the transplanted left lung that continued for 30 minutes. Upon isolation, ventilator settings were changed to a tidal volume of $5 \mathrm{ml} / \mathrm{kg}$ (equivalent to approximately $10 \mathrm{~mL} / \mathrm{kg}$ on the isolated left lung) and the rate was increased to maintain minute ventilation. Upon conclusion of the $30-$ minute isolated reperfusion period, additional assessment of the recipient swine and the transplanted donor lung was performed by way of final arterial blood gas, pulmonary function, and PA catheter measurements before explantation of the transplanted lung.

\section{Cytokine Measurement}

Bronchoalveolar lavage (BAL) of the upper lobe of the left lung was performed immediately after explantation in all groups using $40 \mathrm{~mL}$ normal saline. BAL samples were 
centrifuged at $1800 \mathrm{rpm}$ for 8 minutes and the supernatant was then stored at $-80^{\circ} \mathrm{C}$.

Quantification of cytokine levels in BAL fluid was assessed using a commercially available porcine cytokine multiplex immunoassay kit (RayBiotech, Norcross, GA).

\section{Histopathology and Lung Injury Severity Score}

The lower lobe was fixed in $10 \%$ buffered formalin via tracheal inflation to 25 $\mathrm{cmH}_{2} 0$. Three tissue specimens were obtained from standardized locations within the lung parenchyma with subsequent paraffin embedding and hematoxylin-eosin staining. Lung sections were blindly assessed by a lung pathologist and graded according to total neutrophil counts per high power field, the extent of alveolar edema, and the degree of interstitial infiltration. A score on a scale of 0-3 was assigned for each section using previously reported criteria[109]: neutrophils per high powered field (score: $0=<5,1=6-10$, $2=11-20,3=>20$ ), alveolar edema (score: $0=<5 \%, 1=6-25 \%, 2=26=50 \%, 3=>50 \%$ ), and interstitial infiltration (score: $0=$ none, $1=$ minimal, $2=$ moderate, $3=$ severe), and a composite score was obtained by summation of these three criteria (0-9). The average of the three sample values for each variable was obtained for group comparisons.

\section{Statistical Analysis}

All experimental methodology was designed to test the null hypothesis that no significant differences in the degree of injury would be observed despite different preservation strategies. Independent, pairwise group comparisons were performed utilizing the unpaired Student's $t$ test. Experimental results are reported as mean \pm standard deviation. Significance was defined as $p<0.05$.

\section{Results}

\section{Lung Function}

Although we hypothesized that I-EVLP lungs would function better after transplantation, we instead observed that lung function was significantly improved in the D- 
EVLP group when compared to either CSP or I-EVLP (Figure 20). Compared to the CSP group, final blood oxygenation was significantly higher in the D-EVLP group (D-EVLP: $508.7 \pm 90.4$ vs. CSP: $159.4 \pm 70.1, p<0.001$ ), while mean pulmonary artery pressure (D-EVLP: $22.4 \pm 5.9$ vs. CSP: $30.2 \pm 2.8 \mathrm{mmHg}, p=0.03$ ) and mean airway pressure (D-EVLP: $6.8 \pm 0.8$ vs. CSP: $11.8 \pm 1.0 \mathrm{mmHg}, p<0.001$ ) were significantly reduced. The D-EVLP group also demonstrated significantly higher blood oxygenation (D-EVLP: 508.7ะ90.4 vs. I-EVLP: $228.5 \pm 130.7, p<0.01$ ), significantly lower mean airway pressure (D-EVLP: $6.8 \pm 0.8$ vs. IEVLP: $10.1 \pm 2.6 \mathrm{mmHg}, p=0.03$ ), and reduced mean pulmonary artery pressure (D-EVLP: $22.4 \pm 5.9$ vs. I-EVLP: $29.2 \pm 11.0 \mathrm{mmHg}, p=0.26$ ) compared to the I-EVLP group. Blood oxygenation (I-EVLP: $228.5 \pm 130.7$ vs. CSP: $159.4 \pm 70.1, p=0.33$ ), pulmonary artery pressure (I-EVLP: $29.2 \pm 11.0$ vs. CSP: $30.2 \pm 2.8 \mathrm{mmHg}, p=0.85$ ), and mean airway pressure (I-EVLP: $10.1 \pm 2.6$ vs. CSP: $11.8 \pm 1.0 \mathrm{mmHg}, p=0.27$ ) did not significantly differ between the CSP and IEVLP groups. There were no significant differences observed among all groups in age, weight, mean total anastomotic time, mean arterial pressure, or heart rate (data not shown). 


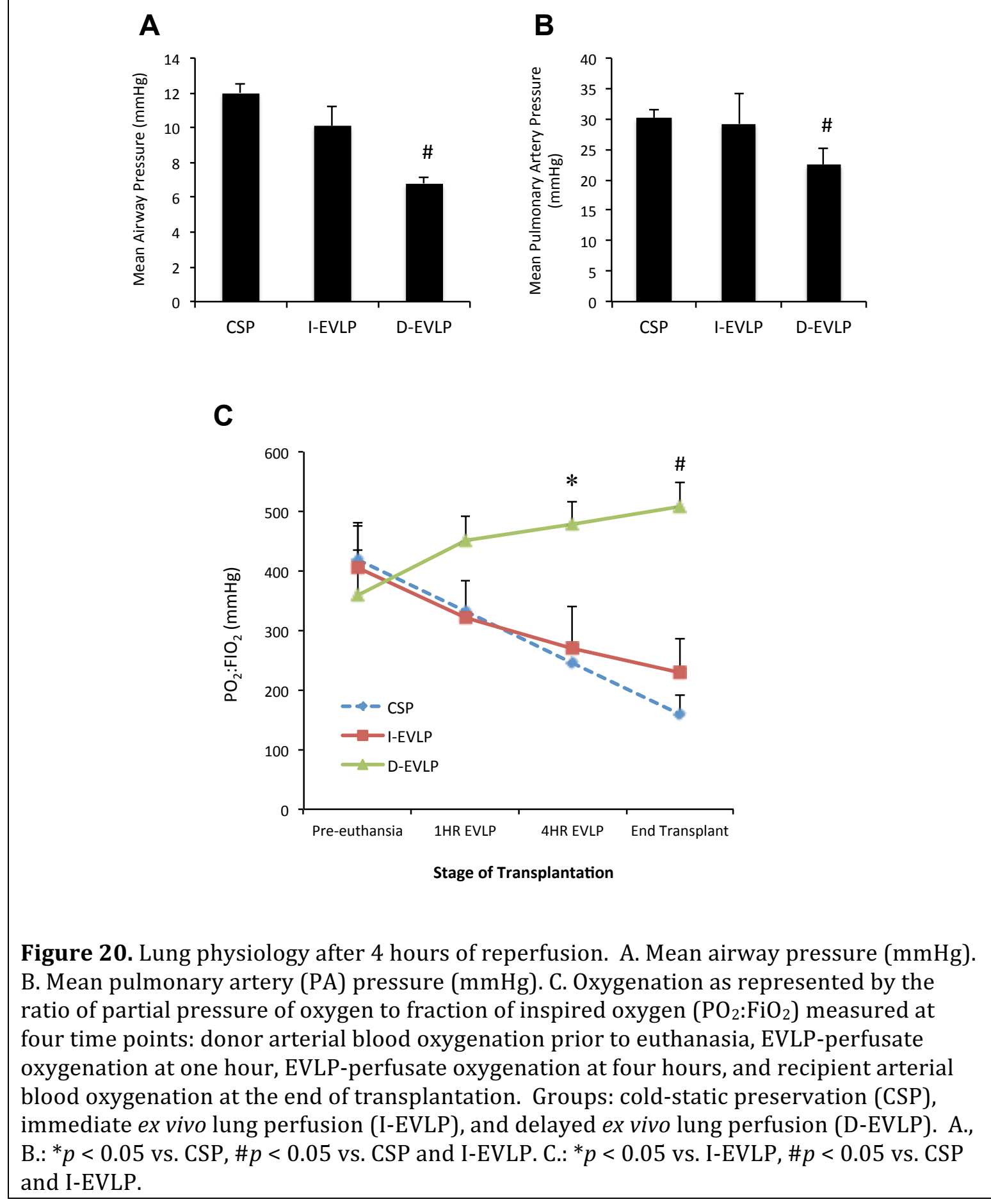

B

All groups had similar pre-euthanasia donor blood oxygenation levels. A divergence was, however, present thereafter as a function of the preservation strategy utilized (Figure 20C). In the CSP group blood oxygenation dropped from $419.4 \pm 108.9$ pre-euthanasia to 159.4 \pm 70.1 post-transplantation. Similarly, pre-euthanasia blood oxygenation in the I-EVLP 
group was high $(406.1 \pm 120.0)$ and perfusate oxygenation levels steadily decreased throughout the EVLP period to a final blood oxygenation level of $228.5 \pm 130.7$ after transplantation. Oxygenation in the D-EVLP group started high $(358.2 \pm 151.1)$ and improved after the period of cold-static preservation and one-hour period of EVLP (450.3 \pm 95.0$)$. Oxygenation levels in the D-EVLP group continued to improve through the end of EVLP (477.6 \pm 88.7$)$ and reached a final blood oxygenation level of 508.7 \pm 90.4 after transplantation.

\section{Proinflammatory Cytokine Expression}

The effect of the various preservation strategies on the expression of proinflammatory cytokines in BAL fluid was assessed at the end of reperfusion (Figure 21). In parallel with the lung function results, the D-EVLP group had significantly decreased expression of IL-1 $\beta$ (D-EVLP: $259.5 \pm 87.3$ vs. CSP: $851.1 \pm 262.8$ pg/ml, $p=0.001$ ), IL-8 (DEVLP: $112.1 \pm 74.8$ vs. CSP: $531.5 \pm 331.4 \mathrm{pg} / \mathrm{ml}, p=0.03$ ), and TNF- $\alpha$ (D-EVLP: $233.2 \pm 84.9$ vs. CSP: $1050.2 \pm 353.8 \mathrm{pg} / \mathrm{ml}, p=0.001$ ) compared to the CSP group. Additionally, the D-EVLP demonstrated significantly decreased IL-1 $\beta$ (D-EVLP: $259.5 \pm 87.3$ vs. I-EVLP: $566.3 \pm 202.1$ $\mathrm{pg} / \mathrm{ml}, p=0.01$ ), IL-8 (D-EVLP: $112.1 \pm 74.8$ vs. I-EVLP: $248.4 \pm 96.3 \mathrm{pg} / \mathrm{ml}, p=0.04$ ), and TNF- $\alpha$ (D-EVLP: $233.2 \pm 84.9$ vs. I-EVLP: $568.1 \pm 218.2 \mathrm{pg} / \mathrm{ml}, p=0.01$ ) in comparison to the I-EVLP group. As shown in Figure 21, the I-EVLP group had intermediate cytokine levels with significantly decreased expression of TNF- $\alpha(p=0.03)$ compared to CSP group. In addition, IL-1 $\beta(p=0.09)$ and IL-8 $(p=0.10)$ were also decreased, but this did not reach statistical significance. 


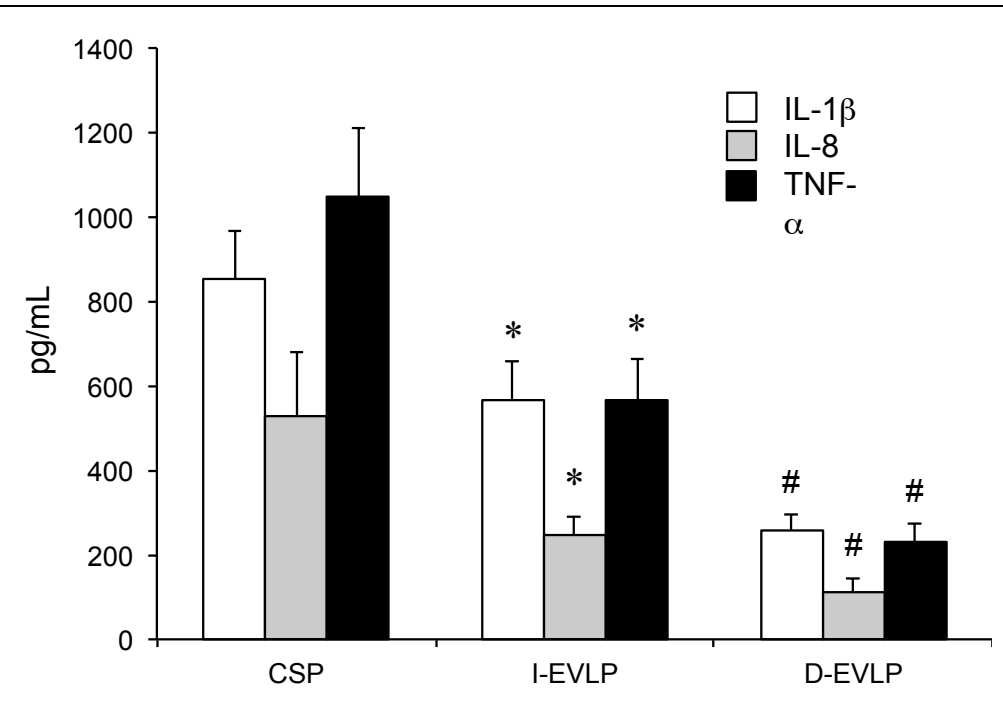

Figure 21. Mean proinflammatory cytokine levels $(\mathrm{pg} / \mathrm{mL})$ in bronchoalveolar lavage fluid at the end of 4 hours of reperfusion. Groups: cold-static preservation (CSP), immediate ex vivo lung perfusion (I-EVLP), and delayed ex vivo lung perfusion (D-EVLP). ${ }^{*} p<0.05$ vs. CSP. $\# p<0.05$ vs. CSP and I-EVLP.

\section{Gross and Histologic Evidence of Lung Injury}

Overall, the D-EVLP group had improved histologic and gross appearance (Figure 22) in addition to decreased lung injury scores (Figure 23) compared to both the CSP and IEVLP groups. Individual parameters of the lung injury severity score demonstrated less injury in the D-EVLP group compared to the CSP group with significantly fewer neutrophils per high-powered field (D-EVLP: $1.6 \pm 0.7$ vs. CSP: $2.6 \pm 0.6, p=0.04$ ) (Figure 23). The D-EVLP group also had decreased alveolar edema (D-EVLP: $0.1 \pm 0.2$ vs. CSP: $0.5 \pm 0.6, p=0.13$ ) and less interstitial infiltrate (D-EVLP: $0.9 \pm 0.6$ vs. CSP: $1.7 \pm 1.0, p=0.12$ ) compared to the CSP group, but these variables did not achieve statistical significance. Additionally, composite lung injury severity scores were less for the D-EVLP group compared to the CSP group (DEVLP: $2.5 \pm 1.2$ vs. CSP: $4.8 \pm 2.0, p=0.06$ ). The D-EVLP group had values less than the I-EVLP group for each parameter yet achieved statistical significance only for the grade of interstitial infiltrate (D-EVLP: $0.9 \pm 0.5$ vs. I-EVLP: $1.7 \pm 0.4, p=0.03$ ). The I-EVLP group did 
not demonstrate a statistically significant reduction in any lung injury parameter in comparison to the CSP group.

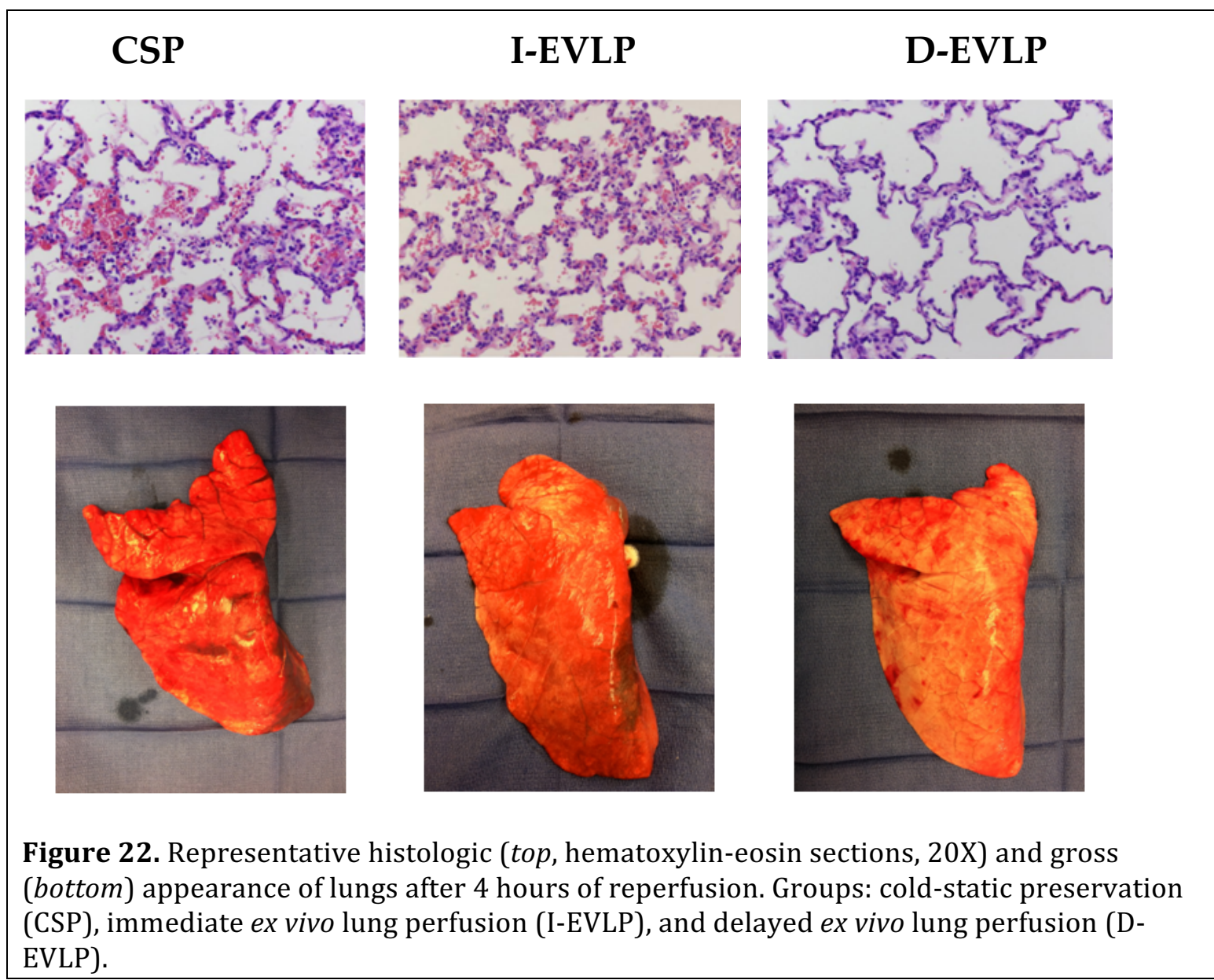




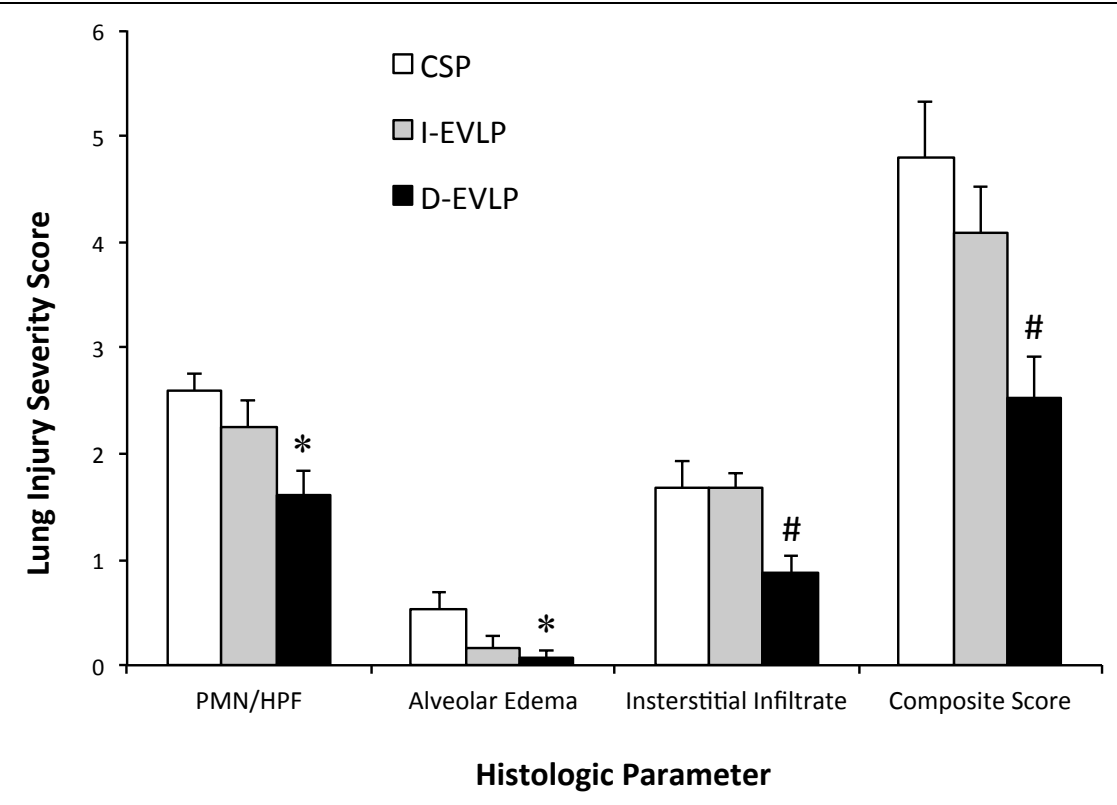

Figure 23. Mean lung injury severity scores by histology. A score on a scale of $0-3$ was assigned for each section: neutrophils per high powered field (score: $0=<5,1=6-10,2=11$ $20,3=>20$ ), alveolar edema (score: $0=<5 \%, 1=6-25 \%, 2=26=50 \%, 3=>50 \%$ ), interstitial infiltration (score: $0=$ none, $1=$ minimal, $2=$ moderate, $3=$ severe), and a composite score obtained by summation of these three criteria (0-9). Groups: cold-static preservation (CSP), immediate ex vivo lung perfusion (I-EVLP), and delayed ex vivo lung perfusion (D-EVLP). ${ }^{*} p$ $<0.05$ vs. CSP. $\# p<0.05$ vs. I-EVLP.

\section{Discussion}

This study used a preclinical porcine lung transplantation model to demonstrate that EVLP can rehabilitate lungs from uncontrolled NHB donors (Maastricht category I) with extended warm ischemic times to an acceptable functional status for successful transplantation. Interestingly, the results of this study, regarding the timing and initiation of EVLP and inclusion of a CSP period, were contrary to our initial hypothesis. These results demonstrate that a combination of 4 hours of CSP followed by 4 hours of normothermic EVLP is significantly more protective than either 4 hours of CSP or 4 hours of immediate EVLP alone. This conclusion is supported by the finding that the D-EVLP group demonstrated significantly improved lung physiology, decreased proinflammatory cytokine expression, decreased neutrophil infiltration and conserved lung histology despite a 
doubling of the preservation period. Although the I-EVLP group did demonstrate decreased proinflammatory cytokine expression and a trend toward improved lung physiology compared to the CSP group, these differences were not significant, unlike those observed with the D-EVLP group. Therefore, while our hypothesis was incorrect, we are encouraged by the finding that excellent post-transplantation lung function can be achieved with uncontrolled NHB donor lungs with extended warm-ischemic times through the use of delayed normothermic EVLP. Additionally, we believe that this study provides the foundation for further research directed toward the goal of expanding the limited human donor pool through the use of uncontrolled NHB donation.

One aspect of this study that warrants further discussion is the use of ATL-1223, a selective A2AR agonist, in the perfusion circuit for both EVLP groups. Our laboratory has extensive experience with the use of selective A2AR agonists in animal models of transplantation[99, 100, 108]. It is well-established that specific A2AR activation decreases the release of TNF- $\alpha$ and other proinflammatory cytokines, down-regulates adhesion molecules (P-selectin, intercellular adhesion molecule-1, and vascular cell adhesion molecule-1), and blocks neutrophil activation and infiltration[100, 137, 138]. We have recently demonstrated in a HB donor porcine transplant model that ATL-1223 attenuates ischemia-reperfusion injury after transplantation[109]. In addition, we have shown that administration of a selective A2AR agonist in the EVLP circuit effectively decreases inflammation and improves lung function in a porcine EVLP non-transplant model[126]. The present study was designed to evaluate the capability for rehabilitation of uncontrolled NHB donor lungs using EVLP. Given the known benefits of ATL-1223 treatment along with the unknown level of lung dysfunction incurred by either the mechanism of donor euthanasia or the subsequent 60-minute warm ischemic time, we included ATL-1223 in the EVLP circuit for both groups to enhance the probability of achieving or exceeding 
acceptable lung function outcomes. We recognize that one limitation of this study is that we are unable to make specific conclusions on the role of ATL-1223, just as we are unable to make any conclusions on the roles of other agents that are currently utilized in standard EVLP protocols. Both the I-EVLP and D-EVLP groups underwent identical exposure to ATL1223 and our results demonstrate that D-EVLP is superior to I-EVLP in the setting of uncontrolled NHB donor lung transplantation.

Conventional wisdom in organ transplantation holds that cold ischemic time is damaging to donor organs, and the United Network for Organ Sharing (UNOS) divides the United States into 11 geographic transplant regions with the goal of minimizing transportation times to limit donor organ preservation periods $[139,140]$. We found that a period of cold-static preservation prior to EVLP was beneficial in optimizing organ function and minimizing inflammation despite the prolonged preservation period this strategy employed. While our findings include an extended preservation period, the benefits of this timeframe are supported in the literature. In a 2011 human clinical EVLP trial, lungs from marginal and NHB Maastricht category III and IV donors underwent EVLP after a period of CSP. EVLP donor lungs yielded equivalent post-transplantation outcomes compared to standard non-marginal donor lungs despite an average of 10.9 hours of total preservation time (versus 6.2 hours for the non-marginal lungs)[21]. Additionally, in the first reported series investigating the use of EVLP in transplantation for standard HB donors, donor lungs underwent CSP followed by EVLP with a total preservation time averaging 17.4 hours, and excellent post-transplantation lung function was achieved in all patients[141].

The benefit of hypothermia is well-established in clinical protocols for organ preservation and protection. Hypothermia is the current clinical standard for neuroprotection during cardiac surgery and is an emerging treatment for patients following cardiac arrest. The benefit of hypothermia is proposed to involve mechanisms beyond the 
slowing of metabolism with colder temperatures[142,143]. Multiple mechanisms for hypothermic protection have been previously described and include: decreased free radical production[144], inhibition of apoptosis[145], suppression of the inflammatory response via inhibition of neutrophil infiltration[146], reduction of lipid peroxidation and leukotriene production[147], and attenuation of the nitric oxide response[148]. Applying this knowledge to the present study, we propose that establishment of lung hypothermia prior to the initiation of EVLP serves to effectively arrest the ongoing tissue damage and inflammatory response associated with the extended warm ischemic time. Subsequent acellular EVLP with the addition of anti-inflammatory compounds including methylprednisolone and ATL-1223 provides a therapeutic environment for tissue rehabilitation prior to transplantation. In contrast, transplantation directly after CSP results in an amplification of the ischemia-reperfusion inflammatory cascade, resulting in subsequent donor organ injury. Similarly, we propose that initiation of EVLP immediately at the conclusion of the warm-ischemic period results in a sustained, hostile proinflammatory environment within the donor lung, ultimately leading to further organ damage. Certainly, further study of the cellular and molecular mechanisms behind the observed results will be required as our study is limited by the absence of mechanistic data both throughout the preservation process and following graft reperfusion. Despite this limitation, these data demonstrate that functional parameters during the EVLP period provide predictive information regarding the potential graft function and candidacy for transplantation. With this understanding, the results of this study demonstrate that the combination of CSP followed by normothermic EVLP effectively reduces the inflammatory response and tissue injury associated with NHB donor lung transplantation.

The findings of the present study support future studies to define the rehabilitative mechanisms and optimal timing of both CSP and EVLP. In the present study, both the 
donor organ harvest and subsequent storage procedure were consistent with current clinical HB donor protocols. In addition, 4 hours of CSP was chosen to approximate the average donor lung cold ischemic time. Therefore, with proper approvals and consent, transplant centers could adopt EVLP as a strategy to further study the assessment and rehabilitation of uncontrolled Maastricht category I and II NHB donor lungs. In addition to further study, our findings support the continued inclusion of CSP in clinical EVLP rehabilitation protocols. As we embark on the clinical adoption of EVLP, our findings provide promise for the inclusion of uncontrolled NHB donors in future human clinical trials for lung transplantation.

In conclusion, delayed ex vivo lung perfusion after a period of cold-static preservation is an effective strategy for the rehabilitation of uncontrolled NHB donor lungs for subsequent transplantation in a preclinical porcine transplant model. Clinical adoption of this lung preservation strategy could be easily applied to current organ procurement protocols. If clinically correlated in human NHB donor lungs, the findings of the present study will lead to improved human lung transplantation by allowing for safe transplantation of uncontrolled NHB donor lungs, thus significantly decreasing the donor organ shortage and saving lives. 
Ex vivo lung perfusion with adenosine $2 A$ receptor agonist improves oxygenation in donor lungs after extended warm-ischemic times

Matthew L. Stone, MD, Nicholas Pope, MD, Lucas G. Fernandez, MD, Victor E. Laubach, PhD, Irving L. Kron, MD

Division of Thoracic and Cardiovascular Surgery, Department of Surgery,

University of Virginia, Charlottesville, VA 


\begin{abstract}
Purpose: Ex vivo lung perfusion (EVLP) provides a technique for donor lung functional assessment, pharmacologic treatment, and rehabilitation. Treatment with adenosine $2 \mathrm{~A}$ receptor (A2AR) agonism has demonstrated promise in the recovery of warm ischemic donor lungs, yet the efficacy of this strategy compared to standard EVLP remains undefined. Thus, the purpose of this study was to evaluate the rehabilitation potential of EVLP with a selective A2AR agonist compared to standard Steen solution alone for donor lungs following 60 minutes of warm ischemia.
\end{abstract}

Methods: Donor lungs from 4 swine underwent standard cold-static preservation (group 1) to serve as a non-treatment control. 20 domestic swine were randomized into two treatment groups ( $n=10$ /group): Steen EVLP (group 2) and Steen EVLP supplemented with a selective A2AR agonist (ATL-1223, group 3). All lungs underwent 60-minutes of no-touch warm ischemia, and groups 2 and 3 underwent EVLP for 4 hours prior to left lung transplantation. Lung function as represented by perfusate or blood oxygenation (PaO2) was obtained during EVLP and post-transplantation. Additionally, hemodynamic parameters were monitored after transplantation throughout the 3.5-hour period of double lung perfusion and final 30-minutes of left lung isolated perfusion and ventilation.

Results: 3 of 10 lungs in group 2 were transplanted without complication and achieved satisfactory recipient single-lung oxygenation levels (mean $\mathrm{PaO2}=416.1 \pm 28.7$ ). The addition of ATL-1223 during EVLP (group 3) resulted in successful transplantation in 3 of 5 lungs (mean $\mathrm{PaO} 2=489.1 \pm 32.1$ ). 5 swine within group 3 experienced death prior to recipient pneumonectomy. Following successful transplantation, EVLP with ATL-1223 resulted in higher PaO2 levels compared to EVLP with Steen solution alone and remained statistically higher than group 1 . No differences were observed for mean airway pressure or pulmonary artery pressure following transplantation between EVLP treatment groups.

Conclusion: EVLP provides a novel method for the assessment and treatment for donor lungs with extended warm ischemic times. A2AR agonism-directed rehabilitation during EVLP results in superior lung function and enhances the rehabilitation potential of EVLP. Warm ischemic times of 60 minutes; however, may lead to irreversible lung injury that is detected by EVLP prior to transplantation. 


\section{Introduction}

Ex vivo lung perfusion (EVLP) provides a novel approach to lung rehabilitation and assessment following donor organ harvest[97]. This technique has inspired renewed interest in the procurement and utilization of lungs following controlled (Maastricht category III) and uncontrolled (Maastricht category IV) cardiac death. Our recent experience has demonstrated that lungs following uncontrolled donation after circulatory death (DCD) may be utilized after 60 minutes of warm ischemia, utilizing a strategy of EVLP with Steen solution $^{\mathrm{TM}}$ (Vitrolife Inc., Denver, CO) and a selective adenosine 2A receptor (A2AR) agonist, ATL-1223 (Dogwood Pharmaceuticals, Charlottesville, VA)[105].

Pharmacologic A2AR receptor agonism is of particular interest within the context of organ rehabilitation given the established effect on the recruitment of anti-inflammatory regulatory $\mathrm{T}$ cells and the suppression of pro-inflammatory pathways involving neutrophils, macrophages, and natural killer T cells $[3,100,149,150]$. Despite these promising findings, the rehabilitative potential of A2AR agonist-directed therapies for lung rehabilitation remains undefined. Thus, the purpose of this study was to evaluate the rehabilitative potential of EVLP and A2AR agonist-directed therapies for DCD lung donation within a preclinical porcine lung transplantation model. We approached this study with the hypothesis that ATL-1223 supplemented Steen solution provides a superior strategy to Steen solution alone for the successful rehabilitation of Maastricht category IV donor lungs.

\section{Methods}

\section{Study Groups}

Mature domestic swine were utilized for the present study, with four swine assigned to the control cold-static preservation group (Group 1) and 20 swine randomized to either Steen EVLP (Group 2, n=10) or Steen + ATL-1223 EVLP (Group 3, n=10) groups. The University of Virginia's Institutional Animal Care and Use Committee reviewed and 
approved the study. Additionally, the presented practices conformed to the "Guide for Care and Use of Laboratory Animals", (National Institutes of Health publication no. 85-23, revised 1985)[105].

\section{Porcine Arrest and Donor Lung Allograft Assignment}

As previously published, a DCD model was utilized that entailed the hypoxic arrest of donor swine followed by a 60-minute period of no-touch warm ischemia[105]. Importantly, no heparin was administered prior to arrest as such systemic administration has both ethical implications for the donation process and such practice has shown no significant impact on outcomes following transplantation[151, 152].

Briefly, swine were anesthetized (50mg/kg ketamine, $5 \mathrm{mg} / \mathrm{kg}$ xylazine), intubated and ventilated on room air. Anesthesia was maintained and lungs were pre-oxygenated for 10 minutes with 1.0 fraction of inspired oxygen $\left(\mathrm{FiO}_{2}\right)($ tidal volume: $8 \mathrm{~mL} / \mathrm{kg}$; respiratory rate: $14-18$ breaths/minutes, and positive end-expiratory pressure (PEEP): $5.0 \mathrm{~cm} \mathrm{H}_{2} 0$ ). Baseline arterial oxygenation concentration was measured prior to ventilator disconnection, endotracheal tube occlusion, and controlled euthanasia by hypoxic arrest. Death was determined at the point of electrocardiographic activity cessation. Swine were maintained at room temperature for 60 minutes prior to resumption of pre-euthanasia ventilator settings and subsequent donor harvest.

Donor lung harvest occurred according to previously published practices[105]. Median sternotomy and great vessel isolation were performed prior to cannulation of the main pulmonary artery with a standard cardioplegia cannula (Sarns, Ann Arbor, MI). Proximal clamp application permitted treatment of the allograft with prostaglandin E1 $(10 \mathrm{mg} / \mathrm{kg})$ with coordinated left atrial appendage ligation, superior and inferior vena cava ligation and antegrade flush of the lungs with $1.5 \mathrm{~L} 4^{\circ} \mathrm{C}$ Perfadex ${ }^{\circledR}$ supplemented with heparin. Visible clot within the left atrium was removed during the flush period. Following 
completion, lungs were harvested at a static tidal volume. The heart was removed on the back table in accordance with clinical practices and an additional retrograde flush of $500 \mathrm{~mL}$ Perfadex ${ }^{\circledR}$ was performed. The EVLP Steen +ATL-1223 group had ATL-1223 supplementation within Perfadex (10mMol) administered during the flush period.

Allografts within the cold-static preservation control group (Group 1) were stored for 4 hours at $4^{\circ} \mathrm{C}$ Perfadex ${ }^{\circledR}$ (Vitrolife Inc., Denver, CO). The EVLP groups underwent a period of 4 hours of cold-static preservation followed by 4 hours of EVLP with either Steen solution (Group 2, n=10) or Steen solution + ATL-1223 (Group 3, n=10). Left donor lungs within all groups were then transplanted into recipient swine as described below.

\section{Porcine EVLP}

EVLP was performed as previously described and in accordance with clinical EVLP protocols $[103,105]$. Standardized cannulation and perfusion equipment were utilized to achieve isolated allograft ex vivo perfusion and ventilation. Antegrade flow was initiated following cannulae fixation at $0.1 \mathrm{~L} / \mathrm{min}$. and titrated to maintain directionality and absence of negative pressure within the left atrial venous return. Steen solution (Vitrolife Inc., Denver, CO) was supplemented with 10,000 IU heparin (APP Pharmaceuticals, Schaumburg, IL), 500mg cefazolin (Apotex Corp., Weston, FL), 500mg methylprednisolone (Pfizer, New York, NY). The ATL-1223 treatment group received treatment by continuous infusion at $3.0 \mathrm{ng} / \mathrm{kg} / \mathrm{min}$. Perfusate re-warming was initiated upon achievement of isolated perfusion to a goal of $37^{\circ} \mathrm{C}$ over a 30 minute period as the flow was titrated to a goal of $40 \%$ the estimated cardiac output $(\mathrm{CO}, 100 \mathrm{~mL} / \mathrm{kg})$. Ventilation was initiated at $32^{\circ} \mathrm{C}$ and at a tidal volume of $8 \mathrm{~mL} / \mathrm{kg}$ (rate: 8 breaths per minute, PEEP: $5 \mathrm{~cm} \mathrm{H}_{2} 0$ ). Deoxygenation was maintained through a membrane oxygenator with the infusion of a standardized gas mixture of $6 \% \mathrm{O}_{2}, 8 \% \mathrm{CO}_{2}$, and $86 \% \mathrm{~N}_{2}$, enabling the measurement of lung oxygenation capacity. Each hour, lungs were ventilated with $1.0 \mathrm{FiO}_{2}$ for 10 minutes to allow assessment 
of perfusate oxygenation by sampling of left atrial return perfusate. Following the 4 hour EVLP period, donor allografts were removed and an antegrade flush was performed with $500 \mathrm{~mL}$ Perfadex prior to back-table preparation of the left lung for subsequent transplantation.

\section{Porcine Left Lung Transplantation}

Donor left lung allografts were implanted according to prior published techniques and clinical practice. A left thoracotomy and pneumonectomy were performed with heparin administration prior to vascular isolation $(5,000 \mathrm{IU})$. The donor allograft was then sewn-in in standard sequence: bronchial, pulmonary artery, pulmonary venous cuff to left atrium. All anastomoses were performed in a continuous running fashion. The vascular and airway occlusive clamps were removed following completion of the anastomoses to establish reperfusion and ventilation.

\section{Lung Physiology}

Recipient allografts were reperfused following transplantation for 4 hours. Serial arterial blood gas assessments in addition to mean arterial pressure and pulmonary arterial pressures were monitored and recorded throughout the reperfusion period. A Swan-Ganz catheter and standard saline-column locked pressure line were utilized to obtain pulmonary arterial and airway pressure measurements, respectively. Following 3.5 hours of reperfusion, the left pulmonary artery was isolated by occlusion of the right pulmonary artery to support isolated allograft perfusion for 30 minutes. During this period of time, the left mainstem bronchus was intubated for isolated ventilation with corresponding adjustment to the tidal volume $(5 \mathrm{~mL} / \mathrm{kg})$ and respiratory rate to maintain minute ventilation. Final measurements for swine not demonstrating ventricular failure or dysrhythmia were obtained by arterial blood gas, pulmonary catheter, and airway pressure measurements. 


\section{Statistics}

Intergroup comparisons were performed with the unpaired Student's $t$ test such as to test the null hypothesis. Data are, thus, presented as mean \pm standard deviation with $p<$ 0.05 defined as a significant difference.

\section{Results}

\section{Study Population}

Pre-euthanasia partial pressures of oxygen in donor swine demonstrated no significant differences among the groups ( $p=0.28$, Figure 24A). Additionally, donor and recipient weights did not significantly differ ( $p=0.35$ and 0.31 , respectively)(Figure 24B-C). Anastomotic times for the transplantation operation did not significantly differ between treatment groups ( $p=0.30$, Figure 24D). Study groups were allocated to support comparison of EVLP treatment groups, as we have previously demonstrated the superiority of EVLP to CSP following 60-minutes of warm ischemia in a porcine transplantation model[103]. Thus, four swine were allocated to the CSP group while 10 swine comprised the Steen EVLP and Steen + ATL-1223 EVLP groups, respectively. Outcomes for all swine randomized within the study are listed in Figure 25. 


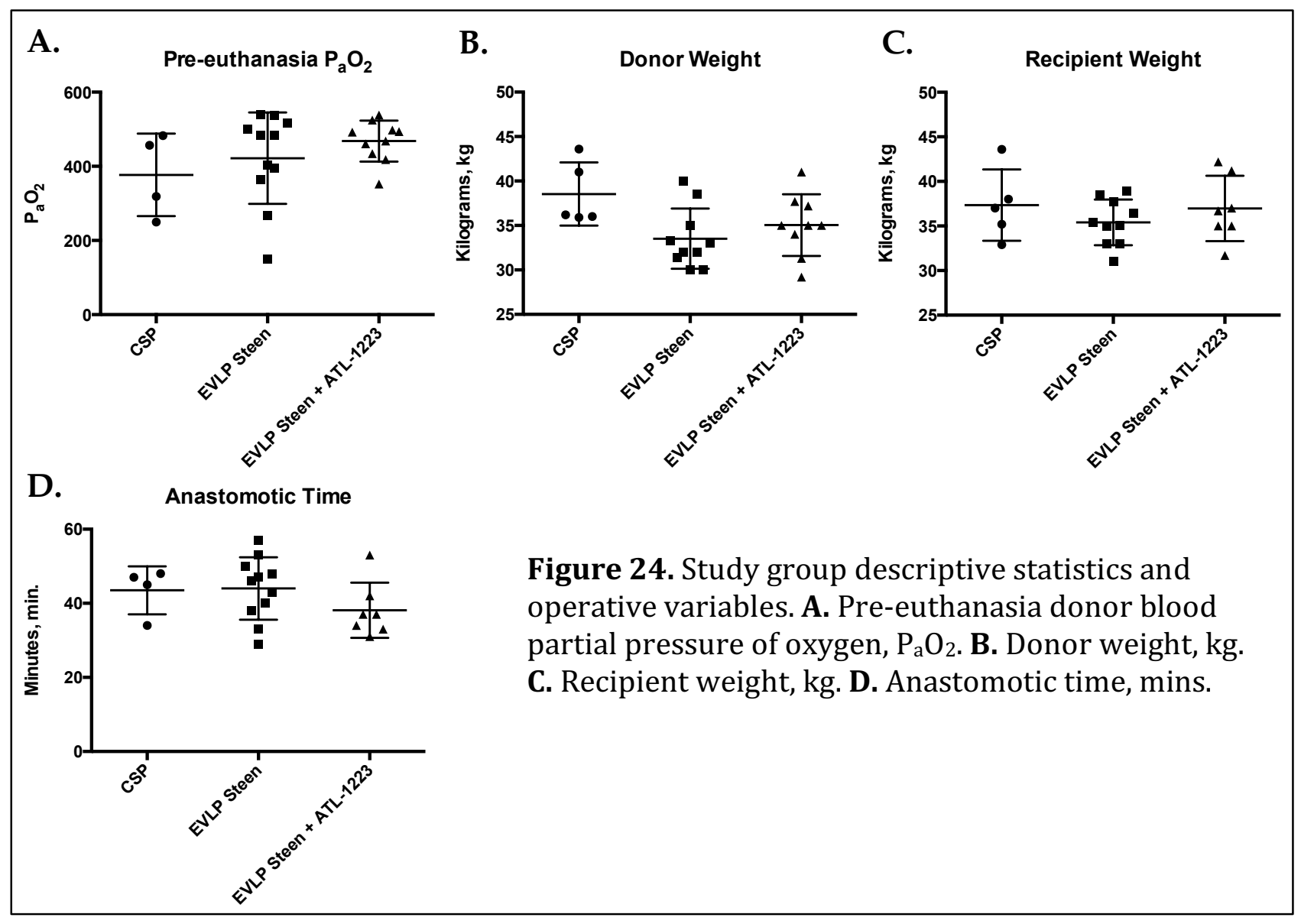




\begin{tabular}{|c|c|}
\hline & Recipient Transplantation \\
\hline & 2 Recipient Transplantation \\
\hline & 3 Recipient death upon anesthesia induction \\
\hline & 4|Hypoxic arrest on single-lung perfusion \\
\hline \multicolumn{2}{|l|}{ Steen EVLP Group } \\
\hline & 1 Recipient Transplantation \\
\hline & 2 Recipient Transplantation \\
\hline & 3 Recipient Transplantation \\
\hline & 4 RV failure with single-lung perfusion \\
\hline & 5 RV failure with single-lung perfusion \\
\hline & 6 Recipient Transplantation \\
\hline & 7 RV failure with single-lung perfusion \\
\hline & $8 \mathrm{RV}$ failure with single-lung perfusion \\
\hline & 9 RV failure with single-lung perfusion \\
\hline & 10|RV failure with single-lung perfusion \\
\hline \multicolumn{2}{|c|}{ Steen + ATL-1223 EVLP Group } \\
\hline & 1 Recipient arrhthmia pre-transplantation \\
\hline & 2 Recipient Transplantation \\
\hline & 3 Recipient malignant hyperthermia pre-transplantation \\
\hline & 4 Recipient Transplantation \\
\hline & 5 Recipient Transplantation \\
\hline & 6 Significant intra-thoracic adhesive disease upon harvest \\
\hline & 7 RV failure with single-lung perfusion \\
\hline & 8 RV failure with single-lung perfusion \\
\hline & 9 Recipient death prior to transplantation \\
\hline & 10|Recipient malignant hyperthermia pre-transplantation \\
\hline
\end{tabular}

Figure 25. All-inclusive outcomes for swine randomized for single-lung transplantation and stratified by donor lung preservation technique.

\section{Lung Physiology}

As demonstrated in Figure 26C and as presented in our prior studies, cold-static preservation results in variable and sub-optimal oxygenation at both the 3.5 hour doublelung perfusion time point and during isolated left lung perfusion following single lung transplantation[105]. Additionally, one swine within the CSP group had right ventricular failure during single lung perfusion that resulted in an early mortality. EVLP groups, as demonstrated in Figures 26A and 26B, maintained stable or improving perfusate oxygenation levels throughout the preservation period. EVLP with Steen solution (Figure 26A) resulted in maintenance of oxygenation throughout the perfusion period with improvement in double-lung oxygenation capacity in a single transplant recipient. Single 
lung oxygenation levels were lower in all recipients that maintained stable hemodynamics and cardiac rhythm during the single lung perfusion and ventilation period. $\mathrm{PaO}_{2}$ as measured at the conclusion of the 4-hour EVLP period in the Steen perfusate was predictive of double lung recipient blood oxygenation following transplantation. The addition of ATL1223 to the Steen perfusate resulted in favorable improvement of oxygenation potential during the EVLP period as demonstrated in Figure 26B. All donor lungs meeting pretransplantation thresholds for transplantation demonstrated stable or improved oxygenation following 3.5 hours of recipient perfusion when compared to levels at the conclusion of the 4 hour EVLP period. With the exception of one recipient that had hemodynamic instability related to right heart failure upon single lung perfusion, all recipient allografts in the Steen + ATL-1223 group had significantly improved oxygenation compared to Steen solution alone (Figure 26).

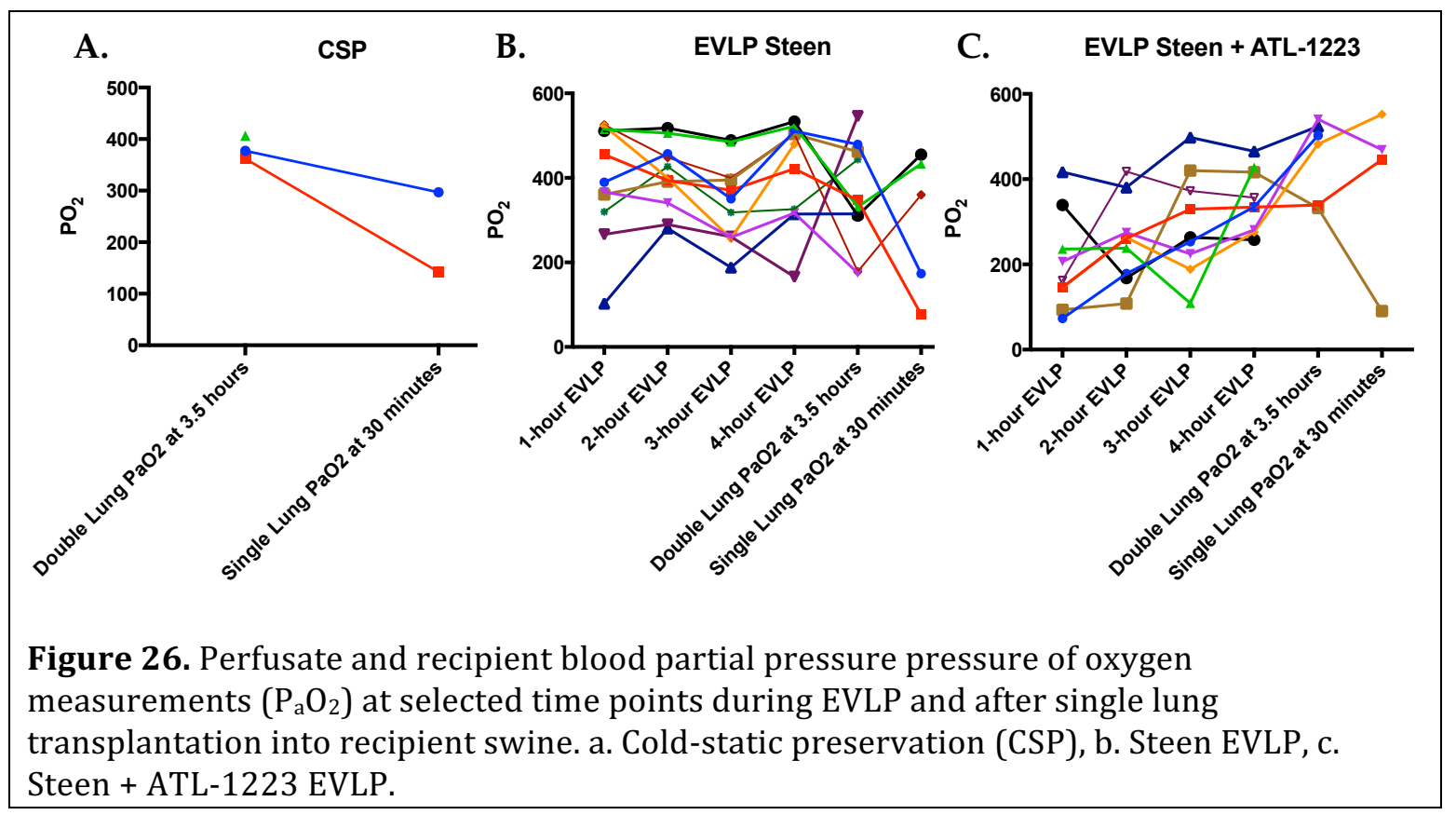

While oxygenation levels achieved in the Steen + ATL-1223 group were higher following transplantation, oxygenation levels early during EVLP at both the 1- and 2-hour 
time points were significantly lower in the ATL-1223 group when compared to Steen solution alone ( $p=0.0029, p=0.0013$, respectively)(Figure 27).

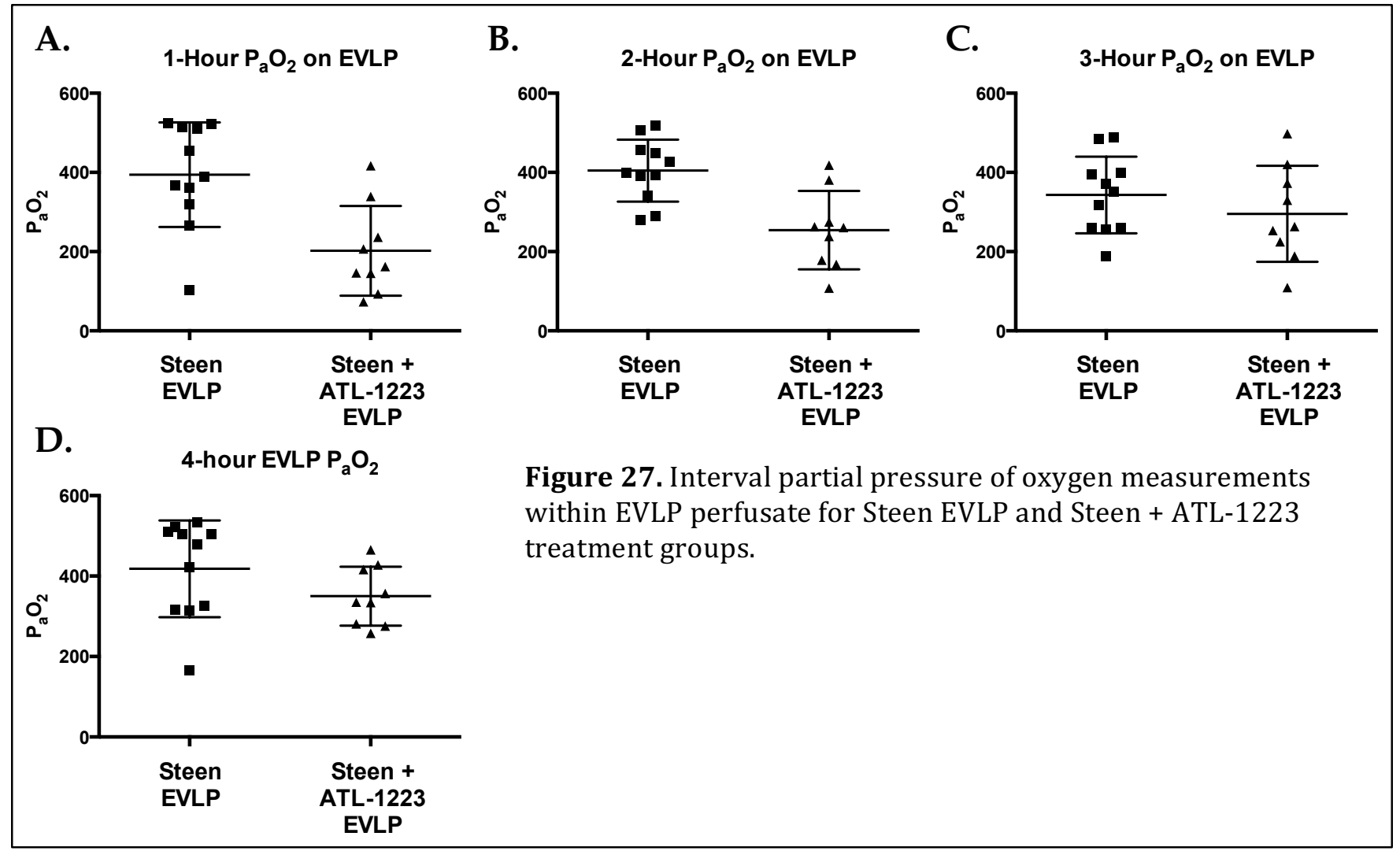

This significant difference was absent at the 3- and 4-hour time points ( $p=0.34$, $p=0.16$, respectively). Following transplantation, significant functional heterogeneity existed between EVLP groups yet lungs perfused with Steen + ATL-1223 achieved the highest oxygenation following four hours of reperfusion (Figure 28). 

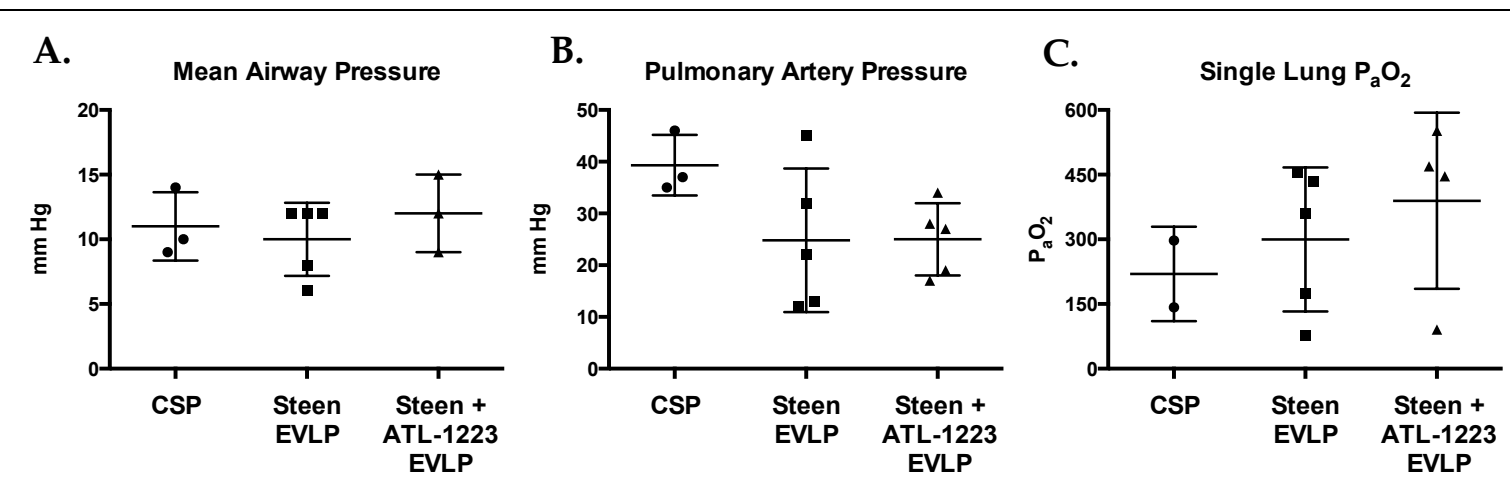

Figure 28. Single lung measurements post-transplantation for mean airway pressure (A), pulmonary artery pressure (B), and partial pressure of oxygen (C).

\section{Discussion}

EVLP has fostered a renewed hope for donor lung allograft rehabilitation and assessment for lung transplantation, demonstrating promise within clinical trials utilizing marginal and high-risk donor lungs for successful transplantation[21]. The rehabilitation of injured DCD lungs is a principal focus for the advancement of this technology. Such a practice promises to provide a potential allograft for the many awaiting lung transplantation yet face an increasing donor organ shortage. The adoption of DCD donors remains limited within the United States, accounting for less than 5\% of lung transplant activity despite the international demonstration of comparable outcomes for DCD and standard brain death donor transplantation[153-156]. Maastricht category III (controlled donation after cardiac death) donor lungs have been utilized internationally for successful transplantation, accounting for $94.8 \%$ of DCD lungs transplanted in 2013-2014, while Maastricht category IV (uncontrolled donation after cardiac death) lungs accounted for only $4 \%$ of DCD lungs transplanted[20]. The purpose of this study was to evaluate the utilization of EVLP and an established A2AR agonist (ATL-1223) in the rehabilitation of Maastricht category IV lungs, as we have previously demonstrated the promise of this allograft 
treatment strategy within a murine EVLP model[157]. The study design enabled the comparison of this strategy to treatment with EVLP and Steen solution alone, as we have previously demonstrated a superiority of delayed EVLP treatment to standard cold-static preservation in DCD lung donation within a preclinical lung transplantation model[105].

The findings of our study demonstrate that Maastricht category IV DCD lung donation carries a significant risk. While supporting a need for objective, selective markers for transplantation utilization, the present results demonstrate that EVLP perfusate oxygenation and allograft function were directly proportional to that achieved posttransplantation. Prior study has supported caution; however, in the utility of functional parameters alone for allograft assessment secondary to the inherent physiologic on-circuit shunt that may occur within an injured allograft[130]. Our prior studies have demonstrated that rapid changes in pulmonary gene expression at the mRNA level occurs during EVLP, offering a potential technique for donor allograft assessment and selection[157]. Consistent with prior study, our experience also demonstrates that EVLP may exacerbate lung injury when lungs have reached a point of irreversible injury. Our data suggest that a decline in function after 4 hours of EVLP is predictive of poor post-transplantation function. Lungs with the capacity for improvement in function on EVLP demonstrated superior posttransplantation function, demonstrating a rehabilitative capability for this strategy. Thus, these data support serial assessments of donor allograft function and perfusate oxygenation during the EVLP period, as the trend of these values rather than absolute values may offer insight into rehabilitative processes for successful transplantation.

A2AR agonism is an established targeted therapy for the amelioration of lung IR injury, with demonstrated effects on macrophage signaling pathways and iNKT cell activation $[3,6,112]$. These findings supported our application of this targeted therapy into the EVLP platform with prior demonstration of rehabilitative promise within a murine 
model of EVLP[157]. We demonstrate that oxygenation during the EVLP period is improved in donor allografts treated with ATL-1223. Paired with this finding is an initial decline in oxygenation within the ATL-1223 group on EVLP followed by a progressive recovery of lung function to levels satisfactory for subsequent transplantation. This trend in perfusate oxygenation warrants further study; however, it may be postulated that the vasodilatory effects of A2A agonism decrease intra-pulmonary shunt such that injured regions of the lung are perfused in treated groups[158]. This vasodilatory effect is exacerbated by hypoxia within coronary arterioles, a mechanism involving cyclic AMP and heat-shock protein 20 signaling pathways[159]. These effects support further study yet offer the potential improvement in the predictive value of functional parameters of lung injury during the EVLP period, through a mechanism of decreased shunt and increased perfusion of injured segments within the donor lung.

While the present study demonstrates promise for the EVLP-mediated rehabilitation of Maastricht category IV lungs, it also demonstrates that many lungs are unrecoverable and that porcine models for transplantation of high-risk donor lungs are limited by labile and often-unpredictable cardiac rhythm profiles within recipient swine. Swine demonstrating a degree of dysrhythmia during single lung perfusion were excluded from further analysis as these animals had altered physiology, specifically significant variability of perfusion pressures within the recipient allograft during single lung perfusion. Paired increases during single lung ventilation and oxygenation of both preload and afterload were not well-tolerated in many swine within the randomized study model. This finding supports donor lung assessment during double-lung perfusion and suggests that single lung perfusion functional measurements may be a function more of cardiac tolerance rather than allograft performance. Paired with this consideration is that lung injury and pulmonary vascular resistance within the selected swine were severe and that the ensuing 
right ventricular failure was a result more of injury than poor cardiac functional reserve. This potential explanation is not supported by the variability within swine, as both injured and non-injured lungs resulted in ventricular dysrhythmias during single lung perfusion and oxygenation. A further limitation to this model is the absence of analysis at the cellular signaling level, as significant heterogeneity existed in donor lung assessment by either biopsy or bronchoalveolar lavage. While each of these techniques was utilized for donor lung assessment, regional variation in lung injury within each allograft introduced a significant sampling bias. Standardized techniques for donor lung assessment are needed, supporting EVLP perfusate analysis and fluorescent labeling as promising measures for comprehensive allograft assessment.

In conclusion, the present study demonstrates that EVLP and ATL-1223 treatment is a promising strategy for the rehabilitation of Maastricht category IV lungs, while also establishing that not all Maastricht category IV lungs are recoverable. Such findings support the continued study of objective parameters for donor allograft assessment during the EVLP period with further evaluation of intra-pulmonary shunt mechanisms and the effect on physiologic measurements of function. Additionally, porcine transplantation protocols utilizing single lung oxygenation and perfusion should be performed with the understanding for potential cardiac dysrhythmias and their effect on measurements of allograft function. Together these findings demonstrate that a responsible advancement of this treatment strategy promises to define markers for allograft usability and carries the potential to address a significant need in the amelioration of donor lung allograft shortages internationally. 


\section{Human EVLP}

The promise of EVLP and ATL-1223 rehabilitative strategies has been recognized with national and international funding support from both the National Institute of Health and the Roche Organ Transplantation Research Fund. This support has enabled a current clinical trial evaluating the efficacy of EVLP and A2AR agonist therapies in donor lung rehabilitation. Presented in Figure 29 are the first human lungs recovered and perfused by EVLP at the University of Virginia with the respective demonstration of improvement in perfusate oxygenation during the EVLP period.
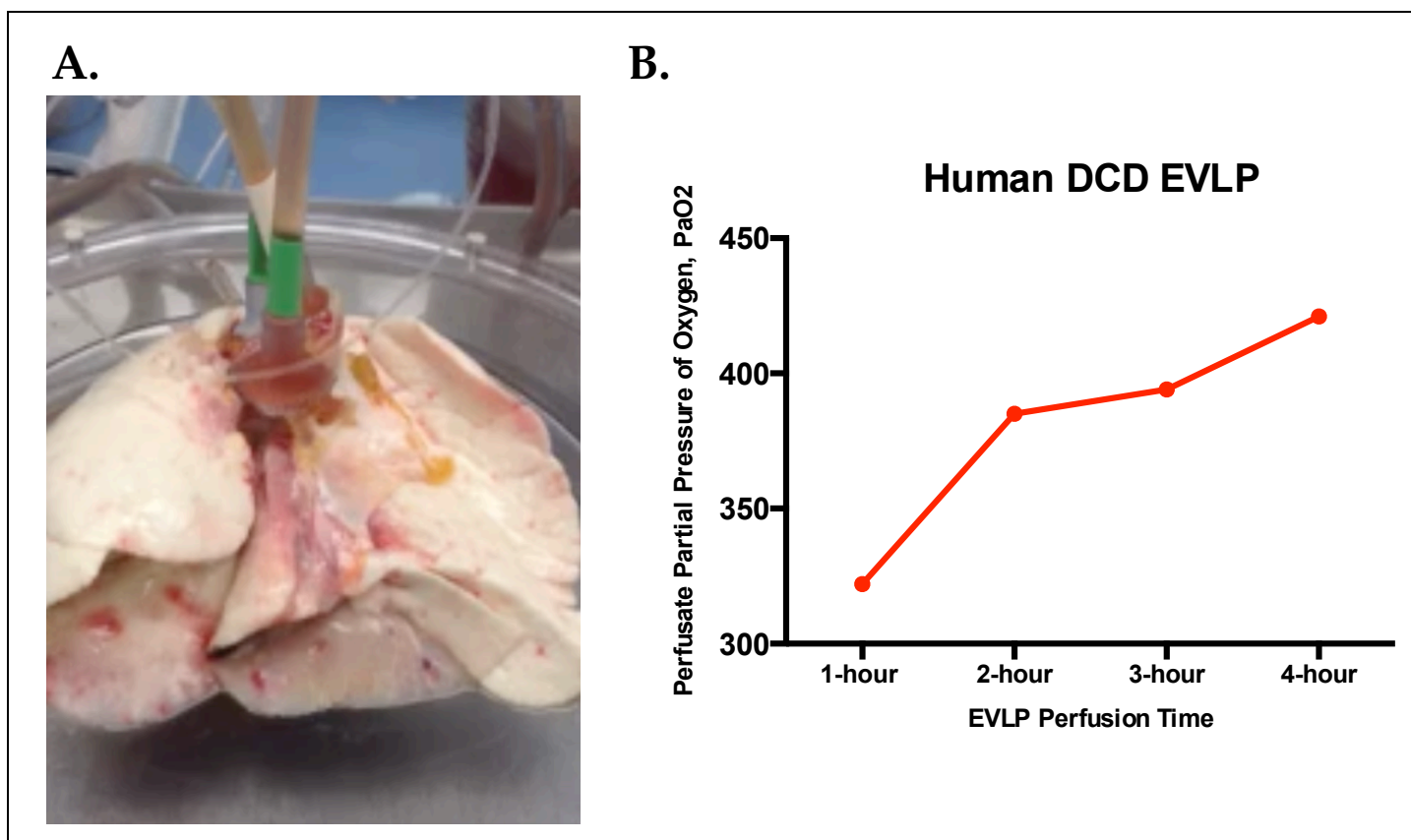

Figure 29. A. First human DCD lungs on EVLP. B. Perfusate PaO2 during EVLP rehabilitation period. 
Concluding Remarks and Future Directives 


\section{Future directives}

\section{Implications for iNKT cell activation}

Prior to our study of early cellular mediators of lung IR injury we identified iNKT cells and IL-17 as principal mediators of IR injury pathogenesis[3]. This study provided the foundational motivation for our evaluation of IL-12 and IL-23 signaling, as these cytokines are implicated in both iNKT cell activation and IL-17 signaling[160-162]. Thus, further study is needed to provide a causal relationship between IL-23 signaling and iNKT cell activation within the context of lung IR injury. As presented in the cytokine profiles for our cellular knockout models, baseline sham IL-17 levels within bronchoalveolar lavage fluid is elevated. This finding limited our determination of the effect of macrophage and dendritic cell depletion on iNKT cell activation and IL-17 release. Thus, future study with the adoptive transfer of IL-23 receptor and IL-12 receptor knockout iNKT cells into J $\alpha$-18 mice (deficient in iNKT cells) would provide added insight into IL-23 and IL-12 mediated iNKT cell activation[163]. Further, mRNA studies and flow cytometry intra-cellular cytokine staining provide potential adjunctive studies to determine transcriptional and translational alterations in IL-23/IL-17 production during lung IR injury. As demonstrated in the S1P agonism studies, cellular recruitment of iNKT does not appear to diminish with the pharmacologic disruption of the examined pro-inflammatory cytokine pathways, yet lung injury is decreased. This finding suggests a decreased activation state for end effector cells of lung IR injury rather than an alteration in cellular recruitment. The in vitro study of dendritic cells and sub-selected pulmonary macrophages in lung IR injury is limited by the significant fragility of these cell populations in vitro and the requirement for sophisticated sorting protocols that threaten cell viability. To potentially circumvent this challenge, a plasmid-encoding construct of the Flt3-ligand was administered by tail vein injection[164]. While the expansion of pulmonary dendritic cells was achieved, cellular clumping and 
viability remained a significant challenge in obtaining adequate purity for in vitro cellular isolates. Refinement of this process; however, would permit the co-culturing of dendritic cells and/or macrophages with iNKT cells to discern potential cross-talk mechanisms between each cell type and the implications of each cell type in iNKT cell activation along with IL-23/IL-17 production. While we have demonstrated feasibility of in vitro hypoxiareoxygenation conditions with iNKT cells, translation of this model to dendritic cells and macrophages resulted in a significant loss of cell viability, which limited validity of in vitro conclusions[165]. Thus, these studies were not included in the presented findings; however, such a model would provide valued insight into the cross-talk mechanisms of dendritic cells and macrophages on iNKT cell activation. While the current study demonstrates that depletion of macrophages independent of dendritic cells provides protection from lung IR injury and provides indirect linkage to IL-23 production and lung IR injury, further analysis directed by these findings provides the potential direct linkage of these cell types and specific end effector cells in IR injury pathogenesis.

\section{Cellular-based perfusion techniques}

The demonstrated findings of the composite EVLP studies support a role for this technology in the assessment and rehabilitation of donor lungs with promise in the rescue of DCD lungs with prolonged warm ischemic time. These findings have significant implications for clinical lung transplantation for both the improvement of outcomes and expansion of a limited donor organ pool. Prior study in renal IR injury has demonstrated that ex vivo treatment of dendritic cells with an A2AR agonist has a protective effect in IR injury[14]. This finding has direct applicability and rational translation to the advancement of EVLP with lung transplantation. The presented conclusions support a protective and rehabilitative role for A2AR agonists in EVLP treatment and it can be theorized that cellbased treatment may provide a more effective and efficient strategy for achievement of 
immunologic tolerance. Direct translation of the presented findings and application of these prior conclusions within renal IR injury would support the evaluation of A2AR agonisttreated dendritic cells and macrophages and the delivery of treated cells by EVLP to the donor allograft. Further, the presented S1P agonism studies provide an additional potential pharmacologic route for dendritic cell signaling alteration through EVLP-directed treatment. The promise of this therapy is that it provides a vehicle for continuous perfusion and cellular monitoring at the epithelial cell level of the donor lung allograft. While an acellular perfusate was utilized in the presented studies, a potential avenue for future study would be to pre-treat the donor organ with recipient dendritic cells and/or macrophages that have been tolerized with either an A2AR agonist or S1P1 receptor agonist. Such study may provide a potential approach to true alteration of the immunologic profile of the donor organ prior to transplantation, limiting both the inherent injury and need for recipient immunosuppression post-implantation and recipient reperfusion.

Markers of injury and assessment of the donor lung

Clinical lung transplantation and donor allograft utilization is currently based upon clinician examination of the donor lung along with a pre-donation determination of oxygenation potential through an arterial blood gas sample. This traditional approach to donor allograft assessment is dependent on additional physiologic parameters and may account for a high percentage of early graft failures secondary to poor allograft selection. EVLP provides the privileged isolated perfusion of the donor allograft, eliminating physiologic variables that may lead to inconsistencies in assessment that threaten both outcomes and utilization. Early study with EVLP has demonstrated an upregulation of IL-6, IL-8, G-CSF, and MCP-1 and downregulation of GM-CSF during acellular EVLP perfusion of rejected human donor lungs when examined in lung biopsy specimens[166]. These findings have provided limited insight into predictive models for lung injury during the EVLP period 
and are potentially limited by heterogeneity within the lung parenchyma that leads to sampling error and variability. Examination of metabolic activity within the lung has also failed to achieve a predictive model of lung injury, as lactate/pyruvate ratios have lacked predictive potential for lung injury post-transplantation[167]. The presented findings demonstrate the real-time measurement of functional parameters during EVLP and provide introductory insight into alterations at the gene expression level within lungs perfused following cardiac death. Each of these avenues afforded promising predictive potential of post-transplantation function in our preclinical porcine transplantation model. Thus, further study to establish objective markers of lung injury promises to provide standards of acceptance for subsequent lung transplantation. Such criteria would limit the transplantation of injured lungs and improve utilization of high-risk donor lungs, providing a significant advancement from current clinical pre-donation standards of oxygenation potential. It is feasible that genetic analysis of both donor and recipient expression profiles could aid in the determination of EVLP-directed therapies to optimize the donor allograft for transplantation and reperfusion periods.

\section{Genetic manipulation of allograft}

Immunologic tolerance is defined as "the specific absence of a destructive immune response to a transplanted tissue in the absence of immunosuppression" and is considered the ultimate benchmark of transplantation pharmacology and cell-based therapies[168]. The presented findings provide insight into cell-based and pharmacologic avenues for donor allograft manipulation, while providing a reproducible vehicle for delivery of such therapies to the isolated donor lung. Adenoviral vector gene delivery has demonstrated promise in the encoding of anti-inflammatory profiles following transplantation when delivered during EVLP to the isolated donor allograft[125]. These early studies demonstrate that alteration of the recipient genetic profile is possible and introduce the concept that 
immunologic tolerance may be achieved by alteration of a donor allograft prior to recipient implantation and reperfusion. Memory T lymphocytes have been demonstrated to be the major barrier to long-term graft survival with the acceleration of rejection responses following rapid infiltration, activation and production of pro-inflammatory mediators[169]. Study of this cell population has demonstrated significant inherent complexity within T cell sub-populations and co-stimulatory molecule expression, implicating T cell subsets both in graft acceptance and acute rejection[169]. Such fluidity within cell populations in the lung demonstrates that both cellular delivery and alteration of recipient pro-inflammatory cytokine profiles offer promise in the achievement of immunologic tolerance and abolishment of deleterious injury following transplantation.

\section{Concluding Comment}

The presented contributions provide foundational insight into the roles for dendritic cells but most predominately macrophages in the early response to lung injury, providing cellular surface markers for implicated cell populations within the lung. Additionally, the absence of IL-23p19 ligand is demonstrated to have a protective advantage in lung IR injury. Pharmacologic modulation of this pro-inflammatory axis is achieved through S1P1 receptor agonism, offering a novel approach to abrogating lung IR injury. Finally, studies within both a murine and pre-clinical porcine EVLP model demonstrate promise for A2AR therapies in the rehabilitation of lungs following donation after cardiac death but most importantly demonstrate that both ex vivo assessment and donor lung treatment are possible. The composite of these studies will continue to inspire personal pursuit of improved outcomes and expansion of lung donation for patients with end stage pulmonary disease. The aim of continued study will be to provide insight into standards for EVLP-directed assessment, while furthering the study of donor allograft genetic manipulation in pursuit of immunologic tolerance for successful transplantation. 


\section{Acknowledgements}

I am grateful for the support of many in my years of study. I would like to thank Drs. Irving Kron, Bruce Schirmer, and Vic Laubach within the Department of Surgery at the University for their mentorship and acceptance of my dedicated study and enrollment during my clinical training as a resident surgeon. I am grateful for each member of my curriculum committee and to Dr. Avril Somlyo for her guidance and encouragement throughout the course of my study and clinical training. I am indebted to the faithful aid of Mr. Tony Herring, Ms. Sheila Hammond, and Ms. Cynthia Dodson and their tireless commitment to the toils of lung transplant operations and logistics navigation. Additionally, I am honored to have shared this experience with intelligent, innovative, and generous collaborating surgeons and scientists. I am humbled to be included in such a community of learning and growth. Lastly, I would like to share my thanksgiving to my family for their understanding, encouragement, and support of this pursuit. I have been blessed in life with the gift of parents that have given of themselves for others. Their belief in me is a constant for which I will be forever grateful. I dedicate this work to their lives with the hope that in some way these studies will provide a foundation for the advancement of our art and the betterment of life for many. 


\section{REFERENCES}

1. Valapour M, Paulson K, Smith JM, et al. OPTN/SRTR 2011 Annual Data Report: lung. Am J Transplant 2013;13 Suppl 1:149-177.

2. Christie JD, Kotloff RM, Ahya VN, et al. The effect of primary graft dysfunction on survival after lung transplantation. Am J Respir Crit Care Med 2005;171:1312-1316.

3. Sharma AK, LaPar DJ, Zhao Y, et al. Natural killer T cell-derived IL-17 mediates lung ischemia-reperfusion injury. Am J Respir Crit Care Med 2011;183:1539-1549.

4. Yang Z, Sharma AK, Linden J, et al. CD4+ T lymphocytes mediate acute pulmonary ischemia-reperfusion injury. J Thorac Cardiovasc Surg 2009;137:695-702; discussion 702.

5. Yang Z, Sharma AK, Marshall M, et al. NADPH oxidase in bone marrow-derived cells mediates pulmonary ischemia-reperfusion injury. Am J Respir Cell Mol Biol 2009;40:375381.

6. Zhao M, Fernandez LG, Doctor A, et al. Alveolar macrophage activation is a key initiation signal for acute lung ischemia-reperfusion injury. Am J Physiol Lung Cell Mol Physiol 2006;291:L1018-1026.

7. Cailhier JF, Sawatzky DA, Kipari T, et al. Resident pleural macrophages are key orchestrators of neutrophil recruitment in pleural inflammation. Am J Respir Crit Care Med 2006;173:540-547.

8. Crapster-Pregont M, Yeo J, Sanchez RL, et al. Dendritic cells and alveolar macrophages mediate IL-13-induced airway inflammation and chemokine production. J Allergy Clin Immunol 2012.

9. Furuhashi K, Suda T, Hasegawa H, et al. Mouse lung CD103+ and CD11bhigh dendritic cells preferentially induce distinct CD4+ T-cell responses. Am J Respir Cell Mol Biol 2012;46:165-172.

10. Husted TL, Blanchard J, Schuster R, et al. Potential role for IL-23 in hepatic ischemia/reperfusion injury. Inflamm Res 2006;55:177-178.

11. Ivanov S, Fontaine J, Paget C, et al. Key role for respiratory CD103(+) dendritic cells, IFN-gamma, and IL-17 in protection against Streptococcus pneumoniae infection in response to alpha-galactosylceramide. J Infect Dis 2012;206:723-734.

12. Liang J, Jung Y, Tighe RM, et al. A macrophage subpopulation recruited by CC chemokine ligand-2 clears apoptotic cells in non-infectious lung injury. Am J Physiol Lung Cell Mol Physiol 2012.

13. Yoshida S, Haque A, Mizobuchi T, et al. Anti-type V collagen lymphocytes that express IL-17 and IL-23 induce rejection pathology in fresh and well-healed lung transplants. Am J Transplant 2006;6:724-735.

14. Li L, Huang L, Ye H, et al. Dendritic cells tolerized with adenosine A(2)AR agonist attenuate acute kidney injury. J Clin Invest 2012;122:3931-3942.

15. Christie JD, Edwards LB, Kucheryavaya AY, et al. The Registry of the International Society for Heart and Lung Transplantation: twenty-seventh official adult lung and heartlung transplant report--2010. J Heart Lung Transplant 2010;29:1104-1118.

16. Hardy JD, Webb WR, Dalton ML, Jr., et al. LUNG HOMOTRANSPLANTATION IN MAN. Jama 1963;186:1065-1074.

17. Yusen RD, Edwards LB, Kucheryavaya AY, et al. The Registry of the International Society for Heart and Lung Transplantation: Thirty-second Official Adult Lung and HeartLung Transplantation Report-2015; Focus Theme: Early Graft Failure. J Heart Lung Transplant 2015;34:1264-1277.

18. Punch JD, Hayes DH, LaPorte FB, et al. Organ donation and utilization in the United States, 1996-2005. Am J Transplant 2007;7:1327-1338. 
19. Valapour M, Skeans MA, Heubner BM, et al. OPTN/SRTR 2013 Annual Data Report: lung. Am J Transplant 2015;15 Suppl 2:1-28.

20. Cypel M, Levvey B, Van Raemdonck D, et al. International Society for Heart and Lung Transplantation Donation After Circulatory Death Registry Report. J Heart Lung Transplant 2015;34:1278-1282.

21. Cypel M, Yeung JC, Liu M, et al. Normothermic ex vivo lung perfusion in clinical lung transplantation. N Engl J Med 2011;364:1431-1440.

22. Bajwa A, Huang L, Ye H, et al. Dendritic cell sphingosine 1-phosphate receptor-3 regulates Th1-Th2 polarity in kidney ischemia-reperfusion injury. J Immunol 2012;189:2584-2596.

23. de Perrot M, Liu M, Waddell TK, et al. Ischemia-reperfusion-induced lung injury. Am J Respir Crit Care Med 2003;167:490-511.

24. Fiser SM, Tribble CG, Long SM, et al. Ischemia-reperfusion injury after lung transplantation increases risk of late bronchiolitis obliterans syndrome. Ann Thorac Surg 2002;73:1041-1047; discussion 1047-1048.

25. Ailawadi G, Lau CL, Smith PW, et al. Does reperfusion injury still cause significant mortality after lung transplantation? J Thorac Cardiovasc Surg 2009;137:688-694.

26. Kreisel D, Krupnick AS, Puri V, et al. Short- and long-term outcomes of 1000 adult lung transplant recipients at a single center. J Thorac Cardiovasc Surg 2011;141:215-222.

27. Wickersham NE, Johnson JJ, Meyrick BO, et al. Lung ischemia-reperfusion injury in awake sheep: protection with verapamil. J Appl Physiol 1991;71:1554-1562.

28. Hoffman SA, Wang L, Shah CV, et al. Plasma cytokines and chemokines in primary graft dysfunction post-lung transplantation. Am J Transplant 2009;9:389-396.

29. McCourtie AS, Farivar AS, Woolley SM, et al. Alveolar macrophage secretory products effect type 2 pneumocytes undergoing hypoxia-reoxygenation. Ann Thorac Surg 2008;86:1774-1779.

30. Vermaelen K, Pauwels R. Accurate and simple discrimination of mouse pulmonary dendritic cell and macrophage populations by flow cytometry: methodology and new insights. Cytometry A 2004;61:170-177.

31. Jakubzick C, Tacke F, Llodra J, et al. Modulation of dendritic cell trafficking to and from the airways. J Immunol 2006;176:3578-3584.

32. Kreisel D, Sugimoto S, Zhu J, et al. Emergency granulopoiesis promotes neutrophildendritic cell encounters that prevent mouse lung allograft acceptance. Blood 2011;118:6172-6182.

33. Li L, Okusa MD. Macrophages, dendritic cells, and kidney ischemia-reperfusion injury. Semin Nephrol 2010;30:268-277.

34. Oppmann B, Lesley R, Blom B, et al. Novel p19 protein engages IL-12p40 to form a cytokine, IL-23, with biological activities similar as well as distinct from IL-12. Immunity 2000;13:715-725.

35. Tan ZY, Bealgey KW, Fang Y, et al. Interleukin-23: immunological roles and clinical implications. Int J Biochem Cell Biol 2009;41:733-735.

36. Li L, Huang L, Vergis AL, et al. IL-17 produced by neutrophils regulates IFN-gammamediated neutrophil migration in mouse kidney ischemia-reperfusion injury. J Clin Invest 2010;120:331-342.

37. Prodinger C, Bunse J, Kruger M, et al. CD11c-expressing cells reside in the juxtavascular parenchyma and extend processes into the glia limitans of the mouse nervous system. Acta Neuropathol 2011;121:445-458.

38. Bar-On L, Jung S. Defining in vivo dendritic cell functions using CD11c-DTR transgenic mice. Methods Mol Biol 2010;595:429-442. 
39. Jung S, Unutmaz D, Wong P, et al. In vivo depletion of CD11c+ dendritic cells abrogates priming of CD8+ T cells by exogenous cell-associated antigens. Immunity 2002;17:211-220.

40. Edelson BT, Kc W, Juang R, et al. Peripheral CD103+ dendritic cells form a unified subset developmentally related to CD8alpha+ conventional dendritic cells. J Exp Med 2010;207:823-836.

41. Hildner K, Edelson BT, Purtha WE, et al. Batf3 deficiency reveals a critical role for CD8alpha+ dendritic cells in cytotoxic T cell immunity. Science 2008;322:1097-1100.

42. Zhao Y, Sharma AK, LaPar DJ, et al. Depletion of tissue plasminogen activator attenuates lung ischemia-reperfusion injury via inhibition of neutrophil extravasation. Am J Physiol Lung Cell Mol Physiol 2011;300:L718-729.

43. Awad AS, Rouse M, Liu L, et al. Activation of adenosine 2A receptors preserves structure and function of podocytes. J Am Soc Nephrol 2008;19:59-68.

44. SP C. Flow Cytometry-Based Analysis and Sorting of Lung Dendritic Cells. In: Schmid I, editor. Flow Cytometry 0 Recent Perspectives2012.

45. Kopf M, Schneider C, Nobs SP. The development and function of lung-resident macrophages and dendritic cells. Nat Immunol 2015;16:36-44.

46. Zhan Y, Xu Y, Seah S, et al. Resident and monocyte-derived dendritic cells become dominant IL-12 producers under different conditions and signaling pathways. J Immunol 2010;185:2125-2133.

47. Li L, Huang L, Sung SS, et al. NKT cell activation mediates neutrophil IFN-gamma production and renal ischemia-reperfusion injury. J Immunol 2007;178:5899-5911.

48. Wilson JM, Kurtz CC, Black SG, et al. The A2B adenosine receptor promotes Th17 differentiation via stimulation of dendritic cell IL-6. J Immunol 2011;186:6746-6752.

49. Anvari F, Sharma AK, Fernandez LG, et al. Tissue-derived proinflammatory effect of adenosine A2B receptor in lung ischemia-reperfusion injury. J Thorac Cardiovasc Surg 2010;140:871-877.

50. Wang J, Ma J, Charboneau R, et al. Morphine inhibits murine dendritic cell IL-23 production by modulating Toll-like receptor 2 and Nod2 signaling. J Biol Chem 2011;286:10225-10232.

51. Yoshiga Y, Goto D, Segawa S, et al. Invariant NKT cells produce IL-17 through IL-23dependent and -independent pathways with potential modulation of Th17 response in collagen-induced arthritis. Int J Mol Med 2008;22:369-374.

52. Ratsimandresy RA, Duvallet E, Assier E, et al. Active immunization against IL-23p19 improves experimental arthritis. Vaccine 2011;29:9329-9336.

53. Granton J. Update of early respiratory failure in the lung transplant recipient. Curr Opin Crit Care 2006;12:19-24.

54. de Perrot M, Young K, Imai Y, et al. Recipient T cells mediate reperfusion injury after lung transplantation in the rat. J Immunol 2003;171:4995-5002.

55. Ross SD, Tribble CG, Gaughen JR, Jr., et al. Reduced neutrophil infiltration protects against lung reperfusion injury after transplantation. Ann Thorac Surg 1999;67:1428-1433; discussion 1434.

56. Stammberger U, Gaspert A, Hillinger S, et al. Apoptosis induced by ischemia and reperfusion in experimental lung transplantation. Ann Thorac Surg 2000;69:1532-1536. 57. Maceyka M, Spiegel S. Sphingolipid metabolites in inflammatory disease. Nature 2014;510:58-67.

58. Chiyo M, Iwata $\mathrm{T}, \mathrm{Webb} \mathrm{TJ}$, et al. Silencing S1P1 receptors regulates collagen-V reactive lymphocyte-mediated immunobiology in the transplanted lung. Am J Transplant 2008;8:537-546. 
59. Garcia JG, Liu F, Verin AD, et al. Sphingosine 1-phosphate promotes endothelial cell barrier integrity by Edg-dependent cytoskeletal rearrangement. J Clin Invest 2001;108:689701.

60. Hla T. Signaling and biological actions of sphingosine 1-phosphate. Pharmacol Res 2003;47:401-407.

61. Sawicka E, Zuany-Amorim C, Manlius C, et al. Inhibition of Th1- and Th2-mediated airway inflammation by the sphingosine 1-phosphate receptor agonist FTY720. J Immunol 2003;171:6206-6214.

62. Okazaki M, Kreisel F, Richardson SB, et al. Sphingosine 1-phosphate inhibits ischemia reperfusion injury following experimental lung transplantation. Am J Transplant 2007;7:751-758.

63. Brinkmann V, Davis MD, Heise CE, et al. The immune modulator FTY720 targets sphingosine 1-phosphate receptors. J Biol Chem 2002;277:21453-21457.

64. Mandala S, Hajdu R, Bergstrom J, et al. Alteration of lymphocyte trafficking by sphingosine-1-phosphate receptor agonists. Science 2002;296:346-349.

65. Ding R, Han J, Tian Y, et al. Sphingosine-1-phosphate attenuates lung injury induced by intestinal ischemia/reperfusion in mice: role of inducible nitric-oxide synthase. Inflammation 2012;35:158-166.

66. Muller HC, Hocke AC, Hellwig K, et al. The Sphingosine-1 Phosphate receptor agonist FTY720 dose dependently affected endothelial integrity in vitro and aggravated ventilatorinduced lung injury in mice. Pulm Pharmacol Ther 2011;24:377-385.

67. Peng X, Hassoun PM, Sammani S, et al. Protective effects of sphingosine 1-phosphate in murine endotoxin-induced inflammatory lung injury. Am J Respir Crit Care Med 2004;169:1245-1251.

68. Zhu R, Snyder AH, Kharel Y, et al. Asymmetric synthesis of conformationally constrained fingolimod analogues--discovery of an orally active sphingosine 1-phosphate receptor type-1 agonist and receptor type-3 antagonist. J Med Chem 2007;50:6428-6435.

69. Sun X, Singleton PA, Letsiou E, et al. Sphingosine-1-phosphate receptor-3 is a novel biomarker in acute lung injury. Am J Respir Cell Mol Biol 2012;47:628-636.

70. Foss FW, Jr., Snyder AH, Davis MD, et al. Synthesis and biological evaluation of gamma-aminophosphonates as potent, subtype-selective sphingosine 1-phosphate receptor agonists and antagonists. Bioorg Med Chem 2007;15:663-677.

71. Awad AS, Ye H, Huang L, et al. Selective sphingosine 1-phosphate 1 receptor activation reduces ischemia-reperfusion injury in mouse kidney. Am J Physiol Renal Physiol 2006;290:F1516-1524.

72. Dudek SM, Camp SM, Chiang ET, et al. Pulmonary endothelial cell barrier enhancement by FTY720 does not require the S1P1 receptor. Cell Signal 2007;19:17541764.

73. Sharma AK, LaPar DJ, Stone ML, et al. Receptor for advanced glycation end products (RAGE) on iNKT cells mediates lung ischemia-reperfusion injury. Am J Transplant 2013;13:2255-2267.

74. Lapar DJ, Hajzus VA, Zhao Y, et al. Acute hyperglycemic exacerbation of lung ischemia-reperfusion injury is mediated by receptor for advanced glycation end-products signaling. Am J Respir Cell Mol Biol 2012;46:299-305.

75. Yanagawa Y, Sugahara K, Kataoka H, et al. FTY720, a novel immunosuppressant, induces sequestration of circulating mature lymphocytes by acceleration of lymphocyte homing in rats. II. FTY720 prolongs skin allograft survival by decreasing T cell infiltration into grafts but not cytokine production in vivo. J Immunol 1998;160:5493-5499. 
76. Anselmo DM, Amersi FF, Shen XD, et al. FTY720 pretreatment reduces warm hepatic ischemia reperfusion injury through inhibition of T-lymphocyte infiltration. Am J Transplant 2002;2:843-849.

77. Tedesco-Silva H, Mourad G, Kahan BD, et al. FTY720, a novel immunomodulator: efficacy and safety results from the first phase $2 \mathrm{~A}$ study in de novo renal transplantation. Transplantation 2005;79:1553-1560.

78. Hirt SW, von Suesskind-Schwendi M, Puehler T, et al. Early administration of FTY720 prevents chronic airway as well as vascular destruction in experimental rat lung transplantation. Transplant Proc 2013;45:783-786.

79. Fryer RM, Muthukumarana A, Harrison PC, et al. The clinically-tested S1P receptor agonists, FTY720 and BAF312, demonstrate subtype-specific bradycardia (S1P(1)) and hypertension (S1P(3)) in rat. PLoS One 2012;7:e52985.

80. Gon Y, Wood MR, Kiosses WB, et al. S1P3 receptor-induced reorganization of epithelial tight junctions compromises lung barrier integrity and is potentiated by TNF. Proc Natl Acad Sci U S A 2005;102:9270-9275.

81. Levkau B, Hermann S, Theilmeier G, et al. High-density lipoprotein stimulates myocardial perfusion in vivo. Circulation 2004;110:3355-3359.

82. Murakami A, Takasugi H, Ohnuma S, et al. Sphingosine 1-phosphate (S1P) regulates vascular contraction via S1P3 receptor: investigation based on a new S1P3 receptor antagonist. Mol Pharmacol 2010;77:704-713.

83. Sobel K, Menyhart K, Killer N, et al. Sphingosine-1-phosphate (S1P) receptor agonists mediate pro-fibrotic responses in normal human lung fibroblasts via S1P2 and S1P3 receptors and Smad-independent signaling. J Biol Chem 2013.

84. Trifilieff A, Fozard JR. Sphingosine-1-phosphate-induced airway hyper-reactivity in rodents is mediated by the sphingosine-1-phosphate type 3 receptor. J Pharmacol Exp Ther 2012;342:399-406.

85. Melendez AJ. Sphingosine kinase signalling in immune cells: potential as novel therapeutic targets. Biochim Biophys Acta 2008;1784:66-75.

86. Schulze T, Golfier S, Tabeling C, et al. Sphingosine-1-phospate receptor 4 (S1P(4)) deficiency profoundly affects dendritic cell function and TH17-cell differentiation in a murine model. FASEB J 2011;25:4024-4036.

87. Matloubian M, Lo CG, Cinamon G, et al. Lymphocyte egress from thymus and peripheral lymphoid organs is dependent on S1P receptor 1. Nature 2004;427:355-360.

88. Graeler M, Shankar G, Goetzl EJ. Cutting edge: suppression of T cell chemotaxis by sphingosine 1-phosphate. J Immunol 2002;169:4084-4087.

89. Wilkerson BA, Argraves KM. The role of sphingosine-1-phosphate in endothelial barrier function. Biochim Biophys Acta 2014;1841:1403-1412.

90. Camp SM, Bittman R, Chiang ET, et al. Synthetic analogs of FTY720 [2-amino-2-(2[4-octylphenyl] ethyl)-1,3-propanediol] differentially regulate pulmonary vascular permeability in vivo and in vitro. J Pharmacol Exp Ther 2009;331:54-64.

91. Ephstein Y, Singleton PA, Chen W, et al. Critical role of S1PR1 and integrin beta4 in HGF/c-Met-mediated increases in vascular integrity. J Biol Chem 2013;288:2191-2200.

92. Finigan JH, Dudek SM, Singleton PA, et al. Activated protein C mediates novel lung endothelial barrier enhancement: role of sphingosine 1-phosphate receptor transactivation. J Biol Chem 2005;280:17286-17293.

93. Schroder M, Richter C, Juan MH, et al. The sphingosine kinase 1 and S1P1 axis specifically counteracts LPS-induced IL-12p70 production in immune cells of the spleen. Mol Immunol 2011;48:1139-1148.

94. $\mathrm{Xu} Y$, Krause A, Limberis M, et al. Low sphingosine-1-phosphate impairs lung dendritic cells in cystic fibrosis. Am J Respir Cell Mol Biol 2013;48:250-257. 
95. Hwang SJ, Kim JH, Kim HY, et al. FTY720, a sphingosine 1-phosphate receptor modulator, inhibits CD1d-restricted NKT cells by suppressing cytokine production but not migration. Lab Invest 2010;90:9-19.

96. Shea BS, Brooks SF, Fontaine BA, et al. Prolonged exposure to sphingosine 1phosphate receptor-1 agonists exacerbates vascular leak, fibrosis, and mortality after lung injury. Am J Respir Cell Mol Biol 2010;43:662-673.

97. Cypel M, Rubacha M, Yeung J, et al. Normothermic ex vivo perfusion prevents lung injury compared to extended cold preservation for transplantation. Am J Transplant 2009;9:2262-2269.

98. Sanchez PG, Bittle GJ, Williams K, et al. Ex vivo lung evaluation of prearrest heparinization in donation after cardiac death. Ann Surg 2013;257:534-541.

99. Gazoni LM, Walters DM, Unger EB, et al. Activation of A1, A2A, or A3 adenosine receptors attenuates lung ischemia-reperfusion injury. J Thorac Cardiovasc Surg 2010;140:440-446.

100. Sharma AK, Laubach VE, Ramos SI, et al. Adenosine A2A receptor activation on CD4+ T lymphocytes and neutrophils attenuates lung ischemia-reperfusion injury. J Thorac Cardiovasc Surg 2010;139:474-482.

101. de Antonio DG, Marcos R, Laporta R, et al. Results of clinical lung transplant from uncontrolled non-heart-beating donors. J Heart Lung Transplant 2007;26:529-534.

102. de Antonio DG, de Ugarte AV. Present state of nonheart-beating lung donation. Curr Opin Organ Transplant 2008;13:659-663.

103. Cypel M, Yeung JC, Hirayama S, et al. Technique for prolonged normothermic ex vivo lung perfusion. J Heart Lung Transplant 2008;27:1319-1325.

104. Dong B, Stewart PW, Egan TM. Postmortem and ex vivo carbon monoxide ventilation reduces injury in rat lungs transplanted from non-heart-beating donors. J Thorac Cardiovasc Surg 2012.

105. Mulloy DP* SM, Crosby IK, LaPar DJ, Sharma AK, Webb DV, Lau CL, Laubach VE, Kron IL. Ex Vivo Rehabilitation of Non-Heart-Beating Donor Lungs in a Preclinical Porcine Model: Delayed Perfusion Results in Superior Lung Function. The Journal of Thoracic and Cardiovascular Surgery 2012; [in press].

106. Nakajima D, Chen F, Yamada T, et al. Reconditioning of lungs donated after circulatory death with normothermic ex vivo lung perfusion. J Heart Lung Transplant 2012;31:187-193.

107. Yeung JC, Cypel M, Waddell TK, et al. Update on donor assessment, resuscitation, and acceptance criteria, including novel techniques--non-heart-beating donor lung retrieval and ex vivo donor lung perfusion. Thorac Surg Clin 2009;19:261-274.

108. Gazoni LM, Laubach VE, Mulloy DP, et al. Additive protection against lung ischemiareperfusion injury by adenosine A2A receptor activation before procurement and during reperfusion. J Thorac Cardiovasc Surg 2008;135:156-165.

109. LaPar DJ, Laubach VE, Emaminia A, et al. Pretreatment strategy with adenosine A2A receptor agonist attenuates reperfusion injury in a preclinical porcine lung transplantation model. J Thorac Cardiovasc Surg 2011;142:887-894.

110. Ross SD, Tribble CG, Linden J, et al. Selective adenosine-A2A activation reduces lung reperfusion injury following transplantation. J Heart Lung Transplant 1999;18:994-1002.

111. Steen S, Kimblad PO, Sjoberg T, et al. Safe lung preservation for twenty-four hours with Perfadex. Ann Thorac Surg 1994;57:450-457.

112. Sharma AK, Linden J, Kron IL, et al. Protection from pulmonary ischemiareperfusion injury by adenosine A2A receptor activation. Respir Res 2009;10:58. 
113. Mas VR, Maluf DG, Stravitz R, et al. Hepatocellular carcinoma in HCV-infected patients awaiting liver transplantation: genes involved in tumor progression. Liver Transpl 2004;10:607-620.

114. Gehrau RC, Archer KJ, Mas VR, et al. Molecular profiles of HCV cirrhotic tissues derived in a panel of markers with clinical utility for hepatocellular carcinoma surveillance. PLoS One 2012;7:e40275.

115. Barash Y, Dehan E, Krupsky M, et al. Comparative analysis of algorithms for signal quantitation from oligonucleotide microarrays. Bioinformatics 2004;20:839-846.

116. Irizarry RA, Hobbs B, Collin F, et al. Exploration, normalization, and summaries of high density oligonucleotide array probe level data. Biostatistics 2003;4:249-264. 117. Team. RDC. R: A language and environment for statistical computing. 2008. 118. Efron B, Tibshirani R. Empirical bayes methods and false discovery rates for microarrays. Genet Epidemiol 2002;23:70-86.

119. Reiner A, Yekutieli D, Benjamini Y. Identifying differentially expressed genes using false discovery rate controlling procedures. Bioinformatics 2003;19:368-375.

120. Mulloy DP, Stone ML, Crosby IK, et al. Ex vivo rehabilitation of non-heart-beating donor lungs in preclinical porcine model: delayed perfusion results in superior lung function. J Thorac Cardiovasc Surg 2012;144:1208-1215.

121. Wallinder A, Ricksten SE, Hansson C, et al. Transplantation of initially rejected donor lungs after ex vivo lung perfusion. J Thorac Cardiovasc Surg 2012;144:1222-1228.

122. Medeiros IL, Pego-Fernandes PM, Mariani AW, et al. Histologic and functional evaluation of lungs reconditioned by ex vivo lung perfusion. J Heart Lung Transplant 2012;31:305-309.

123. Pierre L, Lindstedt S, Hlebowicz J, et al. Is it possible to further improve the function of pulmonary grafts by extending the duration of lung reconditioning using ex vivo lung perfusion? Perfusion 2013;28:322-327.

124. George TJ, Arnaoutakis GJ, Beaty CA, et al. A physiologic and biochemical profile of clinically rejected lungs on a normothermic ex vivo lung perfusion platform. J Surg Res 2013;183:75-83.

125. Yeung JC, Wagnetz D, Cypel M, et al. Ex vivo adenoviral vector gene delivery results in decreased vector-associated inflammation pre- and post-lung transplantation in the pig. Mol Ther 2012;20:1204-1211.

126. Emaminia A, Lapar DJ, Zhao Y, et al. Adenosine A(2)A agonist improves lung function during ex vivo lung perfusion. Ann Thorac Surg 2011;92:1840-1846.

127. Kreisel D, Goldstein DR. Innate immunity and organ transplantation: focus on lung transplantation. Transpl Int 2013;26:2-10.

128. Okusa MD, Linden J, Huang L, et al. A(2A) adenosine receptor-mediated inhibition of renal injury and neutrophil adhesion. Am J Physiol Renal Physiol 2000;279:F809-818.

129. Save S, Mohlin C, Vumma R, et al. Activation of adenosine A2A receptors inhibits neutrophil transuroepithelial migration. Infect Immun 2011;79:3431-3437.

130. Yeung JC, Cypel M, Machuca TN, et al. Physiologic assessment of the ex vivo donor lung for transplantation. J Heart Lung Transplant 2012;31:1120-1126.

131. Yaar R, Jones MR, Chen JF, et al. Animal models for the study of adenosine receptor function. J Cell Physiol 2005;202:9-20.

132. Kootstra G, Daemen JH, Oomen AP. Categories of non-heart-beating donors.

Transplant Proc 1995;27:2893-2894.

133. Gomez-de-Antonio D, Campo-Canaveral JL, Crowley S, et al. Clinical lung transplantation from uncontrolled non-heart-beating donors revisited. J Heart Lung Transplant 2012;31:349-353. 
134. Wigfield CH, Love RB. Donation after cardiac death lung transplantation outcomes. Curr Opin Organ Transplant 2011;16:462-468.

135. Steen S, Sjoberg T, Pierre L, et al. Transplantation of lungs from a non-heart-beating donor. Lancet 2001;357:825-829.

136. Koroskenyi K, Duro E, Pallai A, et al. Involvement of adenosine A2A receptors in engulfment-dependent apoptotic cell suppression of inflammation. J Immunol 2011;186:7144-7155.

137. Linden J. Molecular approach to adenosine receptors: receptor-mediated mechanisms of tissue protection. Annu Rev Pharmacol Toxicol 2001;41:775-787. 138. Lisle TC, Gazoni LM, Fernandez LG, et al. Inflammatory lung injury after cardiopulmonary bypass is attenuated by adenosine $\mathrm{A}(2 \mathrm{~A})$ receptor activation. J Thorac Cardiovasc Surg 2008;136:1280-1287; discussion 1287-1288.

139. Snell GI, Rabinov M, Griffiths A, et al. Pulmonary allograft ischemic time: an important predictor of survival after lung transplantation. J Heart Lung Transplant 1996;15:160-168.

140. Thabut G, Mal H, Cerrina J, et al. Graft ischemic time and outcome of lung transplantation: a multicenter analysis. Am J Respir Crit Care Med 2005;171:786-791.

141. Ingemansson R, Eyjolfsson A, Mared L, et al. Clinical transplantation of initially rejected donor lungs after reconditioning ex vivo. Ann Thorac Surg 2009;87:255-260.

142. Weng Y, Sun S. Therapeutic hypothermia after cardiac arrest in adults: mechanism of neuroprotection, phases of hypothermia, and methods of cooling. Crit Care Clin 2012;28:231-243.

143. Lampe JW, Becker LB. State of the art in therapeutic hypothermia. Annu Rev Med 2011;62:79-93.

144. Globus MY, Busto R, Lin B, et al. Detection of free radical activity during transient global ischemia and recirculation: effects of intraischemic brain temperature modulation. J Neurochem 1995;65:1250-1256.

145. Yang D, Guo S, Zhang T, et al. Hypothermia attenuates ischemia/reperfusioninduced endothelial cell apoptosis via alterations in apoptotic pathways and JNK signaling. FEBS Lett 2009;583:2500-2506.

146. Akriotis V, Biggar WD. The effects of hypothermia on neutrophil function in vitro. J Leukoc Biol 1985;37:51-61.

147. Dempsey RJ, Combs DJ, Maley ME, et al. Moderate hypothermia reduces postischemic edema development and leukotriene production. Neurosurgery 1987;21:177181.

148. Fischer S, Renz D, Wiesnet M, et al. Hypothermia abolishes hypoxia-induced hyperpermeability in brain microvessel endothelial cells. Brain Res Mol Brain Res 1999;74:135-144.

149. Han KL, Thomas SV, Koontz SM, et al. Adenosine A(2)A receptor agonist-mediated increase in donor-derived regulatory $\mathrm{T}$ cells suppresses development of graft-versus-host disease. J Immunol 2013;190:458-468.

150. Reece TB, Ellman PI, Maxey TS, et al. Adenosine A2A receptor activation reduces inflammation and preserves pulmonary function in an in vivo model of lung transplantation. J Thorac Cardiovasc Surg 2005;129:1137-1143.

151. Keshava HB, Farver CF, Brown CR, et al. Timing of heparin and thrombus formation in donor lungs after cardiac death. Thorac Cardiovasc Surg 2013;61:246-250.

152. Wallinder A, Steen S, Liden $\mathrm{H}$, et al. Heparin does not improve graft function in uncontrolled non-heart-beating lung donation: an experimental study in pigs. Eur J Cardiothorac Surg 2013;43:413-419. 
153. Cypel M, Sato M, Yildirim E, et al. Initial experience with lung donation after cardiocirculatory death in Canada. J Heart Lung Transplant 2009;28:753-758.

154. Snell GI, Levvey BJ, Oto T, et al. Early lung transplantation success utilizing controlled donation after cardiac death donors. Am J Transplant 2008;8:1282-1289.

155. Mason DP, Murthy SC, Gonzalez-Stawinski GV, et al. Early experience with lung transplantation using donors after cardiac death. J Heart Lung Transplant 2008;27:561-563. 156. Mason DP, Thuita L, Alster JM, et al. Should lung transplantation be performed using donation after cardiac death? The United States experience. J Thorac Cardiovasc Surg 2008;136:1061-1066.

157. Stone ML, Sharma AK, Mas VR, et al. Ex Vivo Perfusion With Adenosine A2A Receptor Agonist Enhances Rehabilitation of Murine Donor Lungs After Circulatory Death. Transplantation 2015.

158. Hein TW, Belardinelli L, Kuo L. Adenosine A(2A) receptors mediate coronary microvascular dilation to adenosine: role of nitric oxide and ATP-sensitive potassium channels. J Pharmacol Exp Ther 1999;291:655-664.

159. Hedegaard ER, Nielsen BD, Mogensen S, et al. Mechanisms involved in increased sensitivity to adenosine $\mathrm{A}(2 \mathrm{~A})$ receptor activation and hypoxia-induced vasodilatation in porcine coronary arteries. Eur J Pharmacol 2014;723:216-226.

160. Szymczak WA, Sellers RS, Pirofski LA. IL-23 dampens the allergic response to Cryptococcus neoformans through IL-17-independent and -dependent mechanisms. Am J Pathol 2012;180:1547-1559.

161. Doisne JM, Soulard V, Becourt C, et al. Cutting edge: crucial role of IL-1 and IL-23 in the innate IL-17 response of peripheral lymph node NK1.1- invariant NKT cells to bacteria. J Immunol 2011;186:662-666.

162. Moreira-Teixeira L, Resende M, Coffre M, et al. Proinflammatory environment dictates the IL-17-producing capacity of human invariant NKT cells. J Immunol 2011;186:5758-5765.

163. Cui J, Shin T, Kawano T, et al. Requirement for Valpha14 NKT cells in IL-12-mediated rejection of tumors. Science 1997;278:1623-1626.

164. Karsunky H, Merad M, Cozzio A, et al. Flt3 ligand regulates dendritic cell development from Flt3+ lymphoid and myeloid-committed progenitors to Flt3+ dendritic cells in vivo. J Exp Med 2003;198:305-313.

165. Sharma AK, Mulloy DP, Le LT, et al. NADPH oxidase mediates synergistic effects of IL-17 and TNF-alpha on CXCL1 expression by epithelial cells after lung ischemiareperfusion. Am J Physiol Lung Cell Mol Physiol 2014;306:L69-79.

166. Sadaria MR, Smith PD, Fullerton DA, et al. Cytokine expression profile in human lungs undergoing normothermic ex-vivo lung perfusion. Ann Thorac Surg 2011;92:478-484. 167. Koike T, Yeung JC, Cypel M, et al. Kinetics of lactate metabolism during acellular normothermic ex vivo lung perfusion. J Heart Lung Transplant 2011;30:1312-1319. 168. Sachs DH. Transplant tolerance: bench to bedside--26th annual Samuel Jason Mixter Lecture. Arch Surg 2011;146:501-505.

169. Krupnick AS, Lin X, Li W, et al. Central memory CD8+ T lymphocytes mediate lung allograft acceptance. J Clin Invest 2014;124:1130-1143. 